Preprint typeset in JHEP style - HYPER VERSION

hep-ph/

CU-TP-1142

\title{
Radiative Effects on the Chiral Square
}

\author{
Eduardo Pontón and Lin Wang \\ Department of Physics, Columbia University, \\ 538 W. 120th St, New York, NY 10027, USA \\ eponton@phys.columbia.edu, lwang@phys.columbia.edu
}

\begin{abstract}
We consider general field theories in six dimensions, with two of the dimensions compactified on a $T_{2} / Z_{4}$ orbifold. Six-dimensional Weyl fermions propagating on this background give rise to a chiral zero-mode, which makes them interesting for phenomenological applications. The compact two-dimensional space is flat and has three conical singularities. We consider the one-loop structure of these theories, and show that the presence of logarithmic divergences requires the introduction of counterterms precisely at these three singular points. We also show that the corresponding localized operators are rotationally symmetric in the plane of the two extra dimensions, as expected from the geometry about the singularities. We derive the propagators for spin-0, spin- $1 / 2$ and spin- 1 fields in momentum space, in such a way that the appropriate boundary conditions are satisfied. This allows us to efficiently calculate loop diagrams in any given model. We give general expressions for the mass splittings among Kaluza-Klein modes within a given level. Our results can also be used to obtain interesting KK-parity preserving interactions among Kaluza-Klein modes. We pay special attention to the components of six-dimensional gauge fields that transform as scalars under the four-dimensional Lorentz group. These states provide a characteristic signature for these scenarios. In particular, we find that they can easily be the lightest particles in the Kaluza-Klein spectrum.
\end{abstract}

KEYWORDS: extra dimensions; field-theory orbifolds; conical singularities. 


\section{Contents}

1. Introduction 2

2. The Scalar Case: Generalities 11

2.1 Propagators and Boundary Conditions 12

2.2 Propagators in Momentum Space 13

2.2.1 Diagonal Propagators 14

2.2.2 Kaluza-Klein Mixing 15

2.3 KK-Number violating Structure due to Localized Operators 15

3. Chiral Fermions 18

田. Gauge fields 19

4.1 The Spin-1 Components 20

4.2 The Spin-0 Components 20

4.3 Faddeev-Popov Ghosts 21

5. Radiative Corrections 21

5.1 Gauge Boson Two-Point Function 21

5.1 .1 Scalar Matter 22

5.1.2 Fermionic Matter 26

5.1 .3 Gauge Self-Interactions 27

5.1.4 Mass Shifts and Localized Operators 30

5.2 Fermion Two-Point Function 32

5.2.1 One-loop Diagrams: Spinor Manipulations in 6D 32

5.2.2 Mass Shifts and Localized Operators 34

5.2 .3 Yukawa Interactions 36

5.3 Two-Point Functions of Scalar Fields 37

5.3.1 Gauge Interactions 38

5.3 .2 Localized Operators 41

5.3 .3 Yukawa Interactions 42

5.4 Two-Point Function of the Spinless Adjoints 43

5.4.1 Gauge Interactions 44

5.4.2 Fermions and Spinless Adjoints 48

5.4 .3 Scalars and Spinless Adjoints 48

5.4 .4 Localized operators 50

6. Summary and Conclusions 51 
A. Kaluza-Klein Number versus Momentum Space Representations 56

A.1 Diagonal Propagators 56

A.2 Kaluza-Klein Mixing 58

A.3 Useful Identities for the Generalized Functions $\hat{\delta}\left(m, l ; m^{\prime}, l^{\prime} ; n\right)$

B. Tree-level Propagators on the Chiral Square 60

B.1 Chiral Fermions 60

B.2 Gauge Fields: The Spin-1 Components 63

B.3 Gauge Fields: The Spin-0 Components 63

B.4 Feynman Rules for Gauge Interactions 65

\section{Introduction}

Recent years have seen a surge of interest in theories with extra dimensions. This is due, in part, to their potential to explain various unanswered questions in the standard model of particle physics. A second, no less important, reason is that many of these theories are amenable to verification or falsification in the next decade. Our ability to see the new physics and identify it as coming from a higher dimensional structure depends sensitively on which fields can probe the extra dimensions.

One of the most straightforward extensions of the standard model is the assumption that all standard model particles propagate in more than four dimensions, also called Universal Extra Dimensions or UED's. The additional dimensions are compact and would manifest themselves in Kaluza-Klein (KK) towers associated with each and every standard model field. These scenarios naturally contain a dark matter candidate, which can account for the observed dark matter energy density provided the compactification scale is around the electroweak (EW) scale [1]. The six-dimensional case has a number of additional attractive properties. The requirements of anomaly cancellation and fermion mass generation lead to the prediction that the number of fermion generations is a multiple of three [2]. Also, an exact discrete symmetry of the compactified theory provides a natural explanation for the stability of matter, even if baryon number

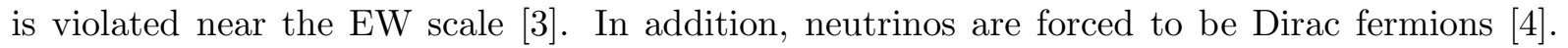
Six-dimensional theories have also been considered in [5].

The phenomenology of the UED scenarios is rather interesting, and the five-dimensional case has received considerable attention [6]. The interactions arising from bulk operators preserve KK-number, which is closely related to momentum conservation in the extra dimensions. An important consequence is that the heavy modes can only be pair produced by such interactions and the effective low-energy theory is simply the standard model, up to loop effects. As a result, the bounds on the compactification scale are of order a few hundred $\mathrm{GeV}$ [7], and the KK states should be accessible in high-energy collider experiments. It is essential to notice that a successful phenomenology can only be obtained when the compact space contains singularities, that allow 
for a chiral low-energy theory. Such singularities can support operators that induce couplings among KK modes not induced by the bulk interactions [8]. Equally important is the fact that they give the leading contribution to the mass splittings among the states within a given KK level. For these reasons, the localized operators are essential in determining the phenomenology of these scenarios. Other studies emphasizing the role of localized terms in extra dimensional scenarios have appeared in [9].

In this paper we consider field theories in six dimensions. Our aim is to understand in detail the quantum structure of these theories, in particular with regard to operators localized at the singular points. We assume a flat spacetime background and that two of the six dimensions are compactified on a "chiral square", as described in [10, 11]. Related studies have appeared in [12, 13]. The chiral square compactification has the following simple description: starting from a two dimensional square region, adjacent sides of the square are identified in pairs. This can be contrasted with the torus construction where opposite sides are identified. The "chiral" square has the topology of a two-dimensional sphere, but the "curvature" is localized at three conical singularities. We will assign them coordinates $\left(x^{4}, x^{5}\right)=(0,0),(L, L)$ and $(0, L) \sim(L, 0)$. The first two singularities have a deficit angle of $3 \pi / 2$, while the latter has a deficit angle of $\pi$. These conical singularities play an important role in determining the physics of these scenarios.

Fields propagating on the chiral square background can belong to four different classes, that may be characterized by the boundary conditions imposed on the sides of the fundamental square region:

$$
\Phi\left(x^{\mu}, y, 0\right)=e^{i n \pi / 2} \Phi\left(x^{\mu}, 0, y\right), \quad n=0,1,2 \text { or } 3
$$

where $x^{\mu}$ are coordinates for the non-compact dimensions and $0 \leq y \leq L$ parametrizes one pair of identified sides of the square. A similar condition holds for the second pair (see Ref. [10] for further details). In addition, the derivatives normal to the "edges" of the square satisfy the "smoothness" condition

$$
\left.\partial_{5} \Phi\right|_{\left(x^{4}, x^{5}\right)=(y, 0)}=-\left.e^{i n \pi / 2} \partial_{4} \Phi\right|_{\left(x^{4}, x^{5}\right)=(0, y)} .
$$

We will label the four classes of fields by the integer $n$, appearing in Eq. (1.1), that characterizes the boundary conditions. ${ }^{1}$ It is understood that $n$ is defined modulo 4 . Notice that only those fields that satisfy boundary conditions corresponding to $n=0$ admit a zero-mode, with an associated flat profile. Furthermore, when considering 6D Weyl fermions, $\Psi_{ \pm}$[we use + and to label the $6 \mathrm{D}$ chiralities and reserve left and right to refer to the $4 \mathrm{D}$ chiralities], one finds that their 4D left- and right-handed chiralities obey boundary conditions corresponding to integers that differ by one: $n_{L}^{ \pm}-n_{R}^{ \pm}= \pm 1$, where the sign depends on the $6 \mathrm{D}$ chirality of the fermion in question. Hence, fermions propagating on this space naturally lead to a chiral low-energy theory: at most one of the left- or right-handed chiralities has a zero mode. This compactification is equivalent to a $T^{2} / Z_{4}$ orbifold [10].

\footnotetext{
${ }^{1}$ There is a second category of fields satisfying "twisted" boundary conditions that never give rise to a zeromode, but we do not consider them here. In the orbifold construction, this corresponds to starting with a "torus" with anti-periodic identifications, before moding out by the discrete $Z_{4}$. See [10] for further details.
} 
Gauge fields propagating in six dimensions give rise to 4-dimensional spin-1 fields plus two scalar states per KK level. One linear combinations of these scalar states becomes the longitudinal polarization of the massive spin-1 fields, while the orthogonal combination remains as an additional scalar degree of freedom. This last phenomenon only occurs in six or higher dimensions, and the observation of such scalar states in the adjoint representation of the gauge group may be taken as a signature of the present class of scenarios. Following Ref. [11], we refer to them as "spinless adjoints".

Another important property of these theories is that one can impose a Kaluza-Klein or KK-parity, defined on KK modes by

$$
\Phi^{(j, k)}\left(x^{\mu}\right) \mapsto(-1)^{j+k} \Phi^{(j, k)}\left(x^{\mu}\right),
$$

where $\Phi$ stands for a field of any spin, and $j, k$ are integers labeling the KK level. The KK-parity has a geometrical interpretation as a rotation by $\pi$ about the center of the chiral square.

It is important to keep in mind that higher dimensional field theories should be regarded as effective theories with a cutoff $\Lambda$, above which a more fundamental UV completion is required. Integrating out the (unknown) physics at the scale $\Lambda$ determines, in principle, the coefficients of various operators through a matching calculation. Of course, even if we knew the UV completion, such a calculation could be in practice very difficult to perform. Therefore, in the spirit of effective theories, we simply allow for all operators consistent with the "low-energy" symmetries, and regard their coefficients as free parameters to be determined, if possible, by experiment.

The operators one can write fall in two distinct classes. Bulk operators, such as the kinetic terms for the various bulk fields or the associated gauge interactions, and operators localized at the three singular points mentioned above. The renormalization program for these scenarios requires localized counterterms to absorb divergences in the quantized theory. In fact, we will see by an explicit computation that the necessary localized counterterms reside precisely at the conical singularities, and have the structure

$$
\left[\delta\left(x^{4}\right) \delta\left(x^{5}\right)+\delta\left(L-x^{4}\right) \delta\left(L-x^{5}\right)\right] \mathcal{O}_{1}+\delta\left(x^{4}\right) \delta\left(L-x^{5}\right) \mathcal{O}_{2}
$$

The fact that the operators at $(0,0)$ and $(L, L)$ have identical coefficients is a consequence of KK-parity. Localized operators at $(0, L)$ have coefficients that are, in general, unrelated to those on the previous two conical singularities. Therefore, in the $6 \mathrm{D}$ theory, each type of localized operator is characterized by two parameters. This should be contrasted with the $5 \mathrm{D}$ case, where a single parameter per operator is sufficient.

The operators appearing in $\mathcal{O}_{i}$ have dimensionful coefficients, suppressed by the scale $\Lambda$. The most important ones are those with the lowest dimensionality. These are kinetic terms such as

$$
\mathcal{O}_{i}=-\frac{1}{4} \hat{r}_{A}^{i} F_{\mu \nu} F^{\mu \nu}+\frac{\hat{r}_{\Psi}^{i}}{\Lambda^{2}} i \bar{\Psi} \Gamma^{\mu} D_{\mu} \Psi+\cdots
$$

where $F_{\mu \nu}$ is the field strength of a generic gauge field, and $\Psi$ stands for a generic $6 \mathrm{D}$ fermion. We are assuming that the gauge bulk kinetic term operator has a coefficient $-1 /\left(4 g_{6}^{2}\right)$, where 
$g_{6}$ is the $6 \mathrm{D}$ gauge coupling constant with mass dimension -1, so that the gauge field has mass dimension 1 , as in four dimensions. We also defined dimensionless coefficients $\hat{r}_{A}^{i}$ and $\hat{r}_{\Psi}^{i}$, with $i=1,2$.

The operators in Eq. (1.5) are very important in determining the physics of these scenarios. They give the leading contributions to the mass splittings within states in a given KK-level. They also induce interactions among KK modes that do not arise from bulk operators. In fact, the interactions among KK-modes induced by bulk operators satisfy well defined rules that follow from the integrals over the extra dimensional space of the KK-mode wavefunction profiles. These "tree-level" selection rules are closely related to momentum conservation in the extra dimension, except that a reversing of momentum is allowed and the momenta along the two compact dimensions can be interchanged. We refer to these type of interactions as "KKnumber" preserving. Interactions arising from localized operators, on the other hand, lead to KK-number violating transitions, which are of great phenomenological interest [6, 14]. The only constraint is that they should satisfy the KK-parity symmetry of Eq (1.3).

It is clearly very important to have an idea of how large the corresponding mass splittings and KK-number violating couplings are. As mentioned before, the values of the dimensionless coefficients in Eq. (1.5) at the scale $\Lambda$ should be taken as free parameters. However, the values that are relevant to answer the previous question are those at the scale of the corresponding KK state, which is in general lower than $\Lambda$. Those values can be found by renormalization group (RG) evolution, with the "bare" coefficients at the scale $\Lambda$ providing the initial conditions. The RG running is determined by the physics below $\Lambda$. Furthermore, it leads to a logarithmic enhancement, so that one can expect the "low-energy" contribution to dominate over the "bare" one. ${ }^{2}$ To the extent that the logarithm is sufficiently large, the size of the dimensionless coefficients is set by the physics below $\Lambda$.

In this paper, we will calculate the contribution associated with the KK-modes below $\Lambda$ at one-loop order. It is natural to ask to what extent one can trust the results of a one-loop calculation. To answer this question, it is necessary to be more specific about how to choose the cutoff scale of the theory. A conservative approach is to identify the cutoff $\Lambda$ with the lowest scale where perturbativity is lost in some sector of the theory. For example, if the field content is that of the standard model, and the $4 \mathrm{D}$ effective low-energy theory is identified with the standard model, $\Lambda$ is the scale at which the $S U(3)_{C}$ gauge interactions get strong. To be more precise, we define strong coupling to correspond to the case where the loop expansion breaks down. That is, all loop orders are equally important and there is no small expansion parameter. This criterion provides a way of estimating the dimensionless coefficients of any operator in the theory, when they are expressed in terms of the cutoff scale $\Lambda$, following the rules of Naive Dimensional Analysis or NDA [15]. In extra dimensional theories, the possibility of having operators localized on subspaces of reduced dimensionality requires an extension of the NDA rules as first studied in [16].

\footnotetext{
${ }^{2}$ Of course, in many instances the separation between the cutoff and KK scales may be of order 10, so that the log may be of order just a few.
} 
However, in practical situations there are additional interactions that are weak at the scale $\Lambda$, e.g. gravitational or the electroweak and Yukawa interactions (other than those associated with the top quark). Loops involving such interactions have a natural expansion parameter in terms of the corresponding coupling. It is also natural to assume that bare operators involving, say, only particles interacting through electroweak interactions, have a corresponding suppression. This comments also hold for flavor violating transitions that are suppressed in the standard model. At any rate, based on phenomenological constraints, such an assumption about the size of the coefficients of certain operators induced by the UV completion seems necessary. As long as the assumption is technically natural, in the sense that said size is of the order of loop effects, we are willing to take it as part of the definition of the scenarios we are interested in.

In the absence of a known UV completion for the kind of theories we study here, we assume that the order of magnitude of the coefficients of bulk and localized operators are no larger than the loop induced effects. For the strongly interacting sector, this agrees with the NDA rules. For weakly interacting particles our assumption amounts to the statement that the size of the bare coefficients, induced by the physics that was integrated out at the scale $\Lambda$, is no larger than the effects of the physics in the theory below $\Lambda$, which is well approximated by the lowest order term in the loop expansion.

Thus, there are two qualitatively different cases: for particles that interact strongly, the best one can hope for is to estimate the size of the coefficients of local operators in the higher dimensional theory. The one-loop contribution to localized operators such as those in Eqs. (1.4) and (1.5) for the case of quarks and gluons can only be taken as indicative of the order of magnitude of the effect. Higher orders in the loop expansion give equally important contributions. On the other hand, for weakly interacting particles one can hope that the one-loop computation is a good approximation and the corresponding effects are under control.

There are also finite one-loop effects that contribute to the mass splittings as well as to the KK-number violating interactions. Some of the finite contributions to the mass splittings can be calculated in the context of a simple torus compactification, as done in [6]. These effects are subdominant, not being logarithmically enhanced. However, the finite contributions to certain KK-number violating interactions can be of phenomenological interest. We mention here two important cases: the couplings of KK-parity even states to a pair of zero-mode gauge bosons, and the coupling of KK-parity even spinless adjoints to a pair of zero-mode fermions.

In the first instance, we notice that the couplings of zero-mode gauge bosons are rather constrained by the unbroken $4 \mathrm{D}$ gauge invariance associated with these massless spin-1 fields. To be specific, consider the coupling of a $(1,1)$ KK-gluon to two gluons. The effective 4D operator must take the form of a product of three field strengths, one associated with each of the spin-1 fields. ${ }^{3}$ This effective four-dimensional, KK-number violating operators can arise from

\footnotetext{
${ }^{3}$ Note that after KK decomposition, the cubic terms in the non-abelian gauge kinetic term of Eq. (1.5) naively lead to a vertex between two gluons and a $(1,1)$ state. However, they also lead to mixing between the zero-mode and the heavy KK modes. The unbroken $4 \mathrm{D}$ gauge invariance insures that this system contains a massless state. Furthermore, there exists a basis where both kinetic and mass mixings between this state and the massive ones are absent. Therefore, dimension four operators that would induce a $(0,0)-(0,0)-(1,1)$ vertex do not exist.
} 
localized operators as in Eq. (1.4) with, e.g.,

$$
\mathcal{O}_{1,2} \sim \frac{\hat{r}}{2^{3} \Lambda^{2}} \mathbf{G} \cdot \mathbf{G} \cdot \mathbf{G},
$$

where $\mathbf{G}$ stands for the gluon field strength and the dots denote appropriate contractions both for Lorentz and gauge indices. Using NDA to estimate the dimensionless coefficient, we find $\hat{r} \sim N_{c} / l_{4}$, where $l_{4}=16 \pi^{2}$, and $N_{c}$ is the number of colors. Integrating over the extra dimensions, we find an effective $4 \mathrm{D}$ operator

$$
\frac{1}{2^{3} M_{c}^{2}}\left(\frac{M_{c}}{\Lambda}\right)^{2}\left(\frac{N_{c}}{l_{4}}\right) \mathbf{G}^{(0,0)} \cdot \mathbf{G}^{(0,0)} \cdot \mathbf{G}^{(1,1)},
$$

where we chose the KK scale $M_{c}=1 / R$ as the mass scale suppressing the operator. Although the fields are not canonically normalized, the coefficients of the various kinetic terms are of order $1 / g_{4}^{2}$, where $g_{4}$ is the observed $S U(3)_{C}$ coupling, which is of order one at the KK scale. Therefore, the size of the effect can be read directly from Eq. (1.7).

The ratio $M_{c} / \Lambda$ can be determined by matching the $6 \mathrm{D}$ and $4 \mathrm{D}$ gauge coupling constants. Neglecting the contribution to the kinetic term from the localized gauge operator in (1.5), this is simply $g_{6}^{2} /(\pi R)^{2} \sim g_{4}^{2}=\mathcal{O}(1)$. Since NDA gives $g_{6}^{2} \Lambda^{2} \sim l_{6} / N_{c}$, where $l_{6}=128 \pi^{3}$ is a $6 \mathrm{D}$ loop factor, we find $\left(M_{c} / \Lambda\right)^{2} \sim N_{c} \pi^{2} / l_{6} \sim N_{c} /\left(2 l_{4}\right)$. This suggests that operators such as (1.6) are generated at two-loop order. In fact, this is easy to see from the fact that at one loop only a finite number of states contribute to KK-number violating operators, and the finiteness follows from the corresponding statement in 4D QCD. At higher loop order, one encounters infinite KK sums that require the localized counterterms in Eq. (1.6).

Nevertheless, we expect a non-vanishing, finite one-loop induced vertex between a $(1,1)$ gluon and two zero-mode gluons. This corresponds to an effective $4 \mathrm{D}$ operator as in (1.7), but without the factor $\left(M_{c} / \Lambda\right)^{2}$. This finite effect clearly dominates over the expected contributions from the physics integrated out at the scale $\Lambda$.

The second example where finite effects may play an important role is the coupling of the KK-parity even spinless adjoints to zero-mode fermions or gluons. The coupling to gluons is similar to the couplings of heavy gluons to gluons just discussed. The coupling to fermions arising from localized operators in the $6 \mathrm{D}$ theory proceeds through operators like ${ }^{4}$

$$
\mathcal{O}_{1,2} \sim \frac{\hat{r}^{\prime}}{\Lambda^{2}} \bar{\Psi} \Gamma^{M} \Gamma^{N} \Gamma^{L} \Psi \partial_{L} G_{M N}
$$

where $M, N=0,1, \ldots, 5$ run over the 6 -dimensional Lorentz indices. Again, the NDA estimate corresponds to a two-loop effect, and the physical coupling is dominated by a finite one-loop contribution. Although quite interesting for phenomenological applications, the calculation of such finite effects is beyond the scope of this work.

\footnotetext{
${ }^{4}$ Note that the operator $i \bar{\Psi}_{1} \Gamma^{M} \Gamma^{N} \Psi_{2} G_{M N}$ has lower dimensionality. However, this operator flips chirality and is forbidden by gauge invariance for the standard model field content, unless $\Psi_{1}=\Psi_{2}$, in which case it does not contain two fermion zero-modes.
} 
In this paper we compute the one-loop logarithmically divergent contributions to the localized kinetic terms of scalar, fermion and gauge fields. This allows us to calculate the leading contributions to the mass splittings and to certain KK-number violating interactions such as those between KK-parity even gauge bosons and zero-mode fermions, which are of phenomenological interest [14]. Recall that the power-law divergences renormalize bulk operators and do not induce mass splittings. Our main results for the localized operators are given in Eqs. (5.11), (5.19), (5.38), (5.58), (5.67), (5.78), (5.85), (5.108), (5.109) and (5.110) in the body of the paper, and are summarized in Eqs. (6.4)-(6.7). We give here the mass shifts for fields of various spins, that can be read from those localized operators [see also Tables 1, 国 and 9].

In the following expressions, $g_{4}$ and $\lambda_{4, i}$ are the 4-dimensional gauge and Yukawa couplings, respectively. $C_{2}(F)$ is the eigenvalue of the Casimir operator in the representation of the fields $F=A_{\mu}, \Psi$ or $\Phi$, while $\operatorname{Tr}\left(T^{a} T^{b}\right)=T(F) \delta_{a b}$, where $T^{a}$ are the generators in the representation of the field $F$. We consider $6 \mathrm{D}$ gauge fields which comprise $4 \mathrm{D}$ spin- 1 and spin- 0 components, 6D Weyl fermions that give rise to a zero-mode of any 4D chirality (we do not consider fermions satisfying $n=2$ boundary conditions), and complex $6 \mathrm{D}$ scalar fields satisfying any of the four boundary conditions $n=0,1,2$ or 3 . The $6 \mathrm{D}$ fermions can have any of the two $6 \mathrm{D}$ chiralities, $\Psi_{ \pm}$. Notice that the Yukawa couplings require the presence of fermions with opposite 6D chiralities.

The mass-shifts are different for KK-parity even and KK-parity odd states, as a result of the localized operators at the conical singularity with coordinates $(0, L)$. We obtain:

For KK-parity odd states, $(-1)^{j+k}=-1$ :

- Spin-1 fields:

$$
\frac{\delta M_{j, k}^{A}}{M_{j, k}}=\frac{g_{4}^{2}}{16 \pi^{2}} \ln \frac{\Lambda^{2}}{\mu^{2}}\left[\frac{14}{3} C_{2}(A)-\frac{2}{3} \sum_{\Psi} T(\Psi)+\sum_{\Phi} T(\Phi) \times\left\{\begin{array}{c}
-5 / 12 \\
1 / 12 \\
3 / 12 \\
1 / 12
\end{array}\right] .\right.
$$

- Spinless adjoints:

$$
\frac{\delta M_{j, k}^{S A}}{M_{j, k}}=\frac{g_{4}^{2}}{16 \pi^{2}} \ln \frac{\Lambda^{2}}{\mu^{2}}\left[8 C_{2}(A)-4 \sum_{\Psi} T(\Psi)+\sum_{\Phi} T(\Phi) \times\left\{\begin{array}{c}
13 / 4 \\
-1 / 4 \\
-11 / 4 \\
-1 / 4
\end{array}\right] .\right.
$$

- Spin-1/2 fields:

$$
\frac{\delta M_{j, k}^{\Psi_{+}}}{M_{j, k}}=\frac{1}{16 \pi^{2}} \ln \frac{\Lambda^{2}}{\mu^{2}}\left[4 \sum_{\text {gauge }} g_{4}^{2} C_{2}(\Psi)+\sum_{i}\left|\lambda_{4, i}\right|^{2} \times\left\{\begin{array}{r}
5 / 8 \\
7 / 8 \\
-3 / 8 \\
-9 / 8
\end{array}\right] .\right.
$$

For chirality - fermions, $\Psi_{-}$, the second and fourth lines are exchanged. 
- Spin-0 fields:

$$
\frac{\delta\left(M_{j, k}^{\Phi}\right)^{2}}{M_{j, k}^{2}}=\frac{1}{16 \pi^{2}} \ln \frac{\Lambda^{2}}{\mu^{2}}\left[\sum_{\text {gauge }} g_{4}^{2} C_{2}(\Phi) \times\left\{\begin{array}{c}
15 / 4 \\
15 / 4 \\
7 / 4 \\
15 / 4
\end{array}+\sum_{i}\left|\lambda_{4, i}\right|^{2} \times\left\{\begin{array}{l}
2 \\
0 \\
0 \\
0
\end{array}\right]\right.\right.
$$

In the equations for the spin-1 fields and spinless adjoints the sums run over $6 D$ Weyl fermions, $\Psi_{ \pm}$, that give rise to a zero-mode. The four lines in the sums over the complex $6 \mathrm{D}$ scalars, $\Phi$, list the results for scalars obeying $n=0,1,2$ and 3 boundary conditions, in that order. The terms proportional to $C_{2}(A)$ include the contributions of the complete $6 \mathrm{D}$ gauge multiplet, i.e. both the $4 \mathrm{D}$ spin-1 components, as well as the spinless adjoints. The sums in the equation for the fermions are over its gauge interactions, and Yukawa interactions with scalars obeying any of the four types of boundary conditions. Similar comments apply to the mass-shift of scalars. In this latter case, the four lines refer to the boundary conditions obeyed by the corresponding scalar, ordered as just mentioned. When the scalar satisfies $n=0$ boundary conditions [first line in Eq. (1.12)], there is also a localized bare mass contribution [see Eq. (6.11)]. In these formulae, $\mu$ is the renormalization scale, and should be taken of the order of the scale of the corresponding KK state, e.g. the tree-level mass $M_{j, k}=\sqrt{j^{2}+k^{2}} / R$.

For KK-parity even states, $(-1)^{j+k}=+1$ :

- Spin-1 fields:

$$
\frac{\delta M_{j, k}^{A}}{M_{j, k}}=\frac{g_{4}^{2}}{16 \pi^{2}} \ln \frac{\Lambda^{2}}{\mu^{2}}\left[\frac{17}{3} C_{2}(A)-\frac{2}{3} \sum_{\Psi} T(\Psi)+\sum_{\Phi} T(\Phi) \times\left\{\begin{array}{c}
-1 / 2 \\
1 / 6 \\
1 / 6 \\
1 / 6
\end{array}\right] .\right.
$$

- Spinless adjoints:

$$
\frac{\delta M_{j, k}^{S A}}{M_{j, k}}=\frac{g_{4}^{2}}{16 \pi^{2}} \ln \frac{\Lambda^{2}}{\mu^{2}}\left[9 C_{2}(A)-4 \sum_{\Psi} T(\Psi)+\sum_{\Phi} T(\Phi) \times\left\{\begin{array}{c}
7 / 2 \\
-1 / 2 \\
-5 / 2 \\
-1 / 2
\end{array}\right] .\right.
$$

- Spin-1/2 fields:

$$
\frac{\delta M_{j, k}^{\Psi_{+}}}{M_{j, k}}=\frac{1}{16 \pi^{2}} \ln \frac{\Lambda^{2}}{\mu^{2}}\left[5 \sum_{\text {gauge }} g_{4}^{2} C_{2}(\Psi)+\sum_{i}\left|\lambda_{4, i}\right|^{2} \times\left\{\begin{array}{r}
3 / 4 \\
3 / 4 \\
-1 / 4 \\
-5 / 4
\end{array}\right]\right.
$$

For chirality - fermions, $\Psi_{-}$, the second and fourth lines are exchanged. 
- Spin-0 fields:

$$
\frac{\delta\left(M_{j, k}^{\Phi}\right)^{2}}{M_{j, k}^{2}}=\frac{1}{16 \pi^{2}} \ln \frac{\Lambda^{2}}{\mu^{2}}\left[\sum_{\text {gauge }} g_{4}^{2} C_{2}(\Phi) \times\left\{\begin{array}{c}
11 / 2 \\
15 / 4 \\
0 \\
15 / 4
\end{array}+\sum_{i}\left|\lambda_{4, i}\right|^{2} \times\left\{\begin{array}{l}
2 \\
1 \\
0 \\
1
\end{array}\right]\right.\right.
$$

For scalars satisfying $n=1$ or $n=3$ boundary conditions (second and fourth lines), the Yukawa contribution given here applies when both $6 \mathrm{D}$ fermions in the loop give rise to a zero-mode. If only one of the fermions contains a zero-mode, the Yukawa contribution has the opposite sign [see subsection 5.3.3 for more details].

A nontrivial check of the previous mass-shift formulae can be obtained by considering a supersymmetric theory. As a first example, consider supersymmetric (SUSY) QCD in six dimensions. This theory contains a 6D gauge field and a 6D Weyl fermion. For concreteness assume that the fermion has $6 \mathrm{D}+$ chirality, $\Lambda_{+}$, and that it gives rise to a left handed zero-mode. In $4 \mathrm{D}, N=1$ language these fields arrange themselves into a vector multiplet, $V=\left(A_{\mu}, \lambda_{+L}\right)$, and a chiral multiplet, $H=\left(A_{-}, \lambda_{+R}^{c}\right)$, where $A_{ \pm}=A_{4} \pm i A_{5}$ are the spinless adjoints, and $\Lambda_{+}=\lambda_{+L}+\lambda_{+R}$. The boundary conditions break the higher dimensional supersymmetry down to $4 \mathrm{D}, N=1$ SUSY: $V$ satisfies $n=0$ boundary conditions, while $H$ satisfies $n=1$ boundary conditions. Therefore, the above formulae should predict that $A_{\mu}$ and $\lambda_{+L}$ present the same mass-shift, as well as $A_{+}$and $\lambda_{+R}$. Actually, since at each KK level $\lambda_{+L}$ and $\lambda_{+R}$ combine into a Dirac fermion, the complete $4 \mathrm{D}, N=2$ supermultiplet should present the same mass-shift, a fact that is easy to check from Eqs. (1.9)-(1.11) [and independently from Eqs. (1.13)-(1.15)]. One can also check some of the terms coming from the Yukawa interactions by adding a hypermultiplet, i.e. a $6 \mathrm{D}$ Weyl spinor, $\Psi_{-}$, assumed to have a left-handed zero-mode, and two complex $6 \mathrm{D}$ scalars, $\Phi$ and $\Phi^{c}$. In $4 \mathrm{D}, N=1$ language these decompose into two chiral multiplets, $Q=\left(\Phi, \psi_{-L}\right)$ and $Q^{c}=\left(\Phi^{c}, \psi_{-R}^{c}\right)$, where $\Psi_{-}=\psi_{-L}+\psi_{-R}$. Now $Q$ satisfies $n=0$ boundary conditions, while $Q^{c}$ satisfies $n=3$ boundary conditions. In the SUSY limit, the gauginos interact with the scalars and fermions in the hypermultiplet with Yukawa couplings of strength $\lambda_{4}=\sqrt{2} g_{4}$. Taking this into account, the mass-shifts for the KK parity odd gauge bosons, gauginos and spinless adjoints are all proportional to $4 C_{2}(A)-T(Q)$. Similarly, the mass-shifts for the KK-parity even states are proportional to $5 C_{2}(A)-T(Q)$. To check that the fermion and scalar fields in the hypermultiplet present a common mass-shift requires inclusion of the effects from the trilinear and quartic scalar self-interactions in the scalar mass formulae, which we have not computed. Nevertheless, the mass-shift for the hypermultiplet can be obtained from the fermion mass-shift formulae given above.

Notice that there are a couple of qualitative differences compared to the mass shifts one obtains in a 5-dimensional theory. First, in $6 \mathrm{D}$ the fermions give a negative contribution to the masses of the gauge bosons, whereas in $5 \mathrm{D}$ the fermion contribution vanishes as a result of a cancellation between the left- and right-handed components of the 5D Dirac fermion [6]. In the 6-dimensional case, one can trace the surviving contribution to the existence of additional 
states. For example, the mass shift of the $(2,0)$ states, which play a role akin to the second level states in $5 \mathrm{D}$, receives no contribution from $(1,0)$ states due to a cancellation similar to the $5 \mathrm{D}$ case, but it receives a contribution from the $(1,1)$ states, which have no analog in 5D. A second difference is related to the Yukawa contributions to the fermion masses, due to couplings to scalars with a zero-mode: in $5 \mathrm{D}$ such a contribution is negative, but in $6 \mathrm{D}$ it is found to be positive. The positive sign is special to six dimensions, and originates in the existence of two $6 \mathrm{D}$ chiralities. The Yukawa coupling necessarily involves two fermions of opposite 6D chiralities, and this translates into a relative physical phase that accounts for the previous result. Finally, one can also see that the spinless adjoints receive a negative contribution from their gauge interactions with the fermions, as do the gauge bosons. However, the coefficient in the spinless adjoint formula is larger than for the gauge bosons. As a result, the spinless adjoints are lighter than their spin-1 counterparts. This may have interesting consequences for dark matter, since the lightest KK particle, in the 6D standard model context, is the hypercharge spinless adjoint. It can also affect the collider phenomenology in an interesting way [14].

The general formulae Eqs. (1.9)-(1.16) can be easily applied to various models of interest. As explained before, when applied to strongly interacting particles they should be taken only as indicative of the order of magnitude of the effect. However, when applied to weakly interacting particles, such as the electroweak gauge bosons or leptons, they should reliably give the leading contribution to the corresponding mass shifts.

This paper is organized as follows: in Section 2 we develop the technical ingredients that are necessary to perform the one-loop computation. This requires finding propagators that encode correctly the boundary conditions implied by Eqs. (1.1) and (1.2). We do so in the context of a scalar field theory. We then give the propagators for fermion fields (Section 3) and gauge fields (Section 4). The latter include both the spin-1 and spin-0 components (under the 4D Lorentz group), as well as the ghost fields. Section 5 contains our main results. We compute the one-loop corrections to the gauge boson two-point function in Subsection 5.1, to the fermion two-point function in Subsection 5.2, to the scalar two-point function in Subsection 5.3, and to the two-point functions of the "spinless adjoints" in Subsection 5.4. We summarize and conclude in Section 6. We also include two appendices. In Appendix A we give details on how to relate the KK-number and momentum space representations of the propagators. In Appendix B we give details of the derivation of the 6D propagators associated with fermion and $6 \mathrm{D}$ gauge fields, propagating on the chiral square background.

\section{The Scalar Case: Generalities}

Our first goal is to develop the tools necessary to perform loop calculations in an efficient manner. One approach would be to decompose the bulk fields into a set of Kaluza-Klein modes satisfying the appropriate boundary conditions, and derive the effective four-dimensional theory to read the relevant vertices (given by integrals over KK wavefunctions). These can then be used to calculate any quantity of interest. An alternative approach is to work in momentum space in the extra dimensions, and include the correlations arising from the boundary conditions in the form 
of the propagators. This latter approach has the advantage that the effects of the boundary conditions appear only in the propagators, and are therefore universal. The vertices conserve momentum in the standard sense and can be read as in the $T^{2}$ compactification. We adopt the second approach since it is simpler to generalize to various types of interactions. The first step is then to understand how to encode the boundary conditions in the form of the propagators.

We start by deriving some useful general relations in the context of a scalar field theory. We first write down the general expression for the scalar propagator in "Kaluza-Klein space", where the boundary conditions are manifest. We can then use this representation as a starting point for deriving the momentum space expression of the propagator, that correctly includes the effects of the boundary conditions. We also discuss the generalizations needed in the presence of Kaluza-Klein mixing. This will allow us to understand the structure of the propagator when radiative effects are included.

\subsection{Propagators and Boundary Conditions}

We start from the KK expansion of a 6-dimensional scalar field

$$
\Phi_{n}\left(x^{\mu} ; z\right)=\frac{1}{L} \sum_{j, k}^{\prime} \phi^{(j, k)}\left(x^{\mu}\right) f_{n}^{(j, k)}(z),
$$

where we use the shorthand notation $z=\left(x^{4}, x^{5}\right)$. These coordinates range over the fundamental

square $0 \leq x^{4}, x^{5} \leq L$. The KK wavefunctions, $f_{n}^{(j, k)}(z)$, satisfy the boundary conditions appropriate for the "chiral square", as derived in Ref. [10]. They may be written as

$$
f_{n}^{(j, k)}(z)=\frac{1}{2\left(1+\delta_{j, 0} \delta_{k, 0}\right)}\left[h^{(j, k)}(z)+e^{i \theta} h^{(k,-j)}(z)+e^{2 i \theta} h^{(-j,-k)}(z)+e^{3 i \theta} h^{(-k, j)}(z)\right],
$$

in terms of the momentum space wavefunctions

$$
h^{(j, k)}(z)=e^{i\left(j x^{4}+k x^{5}\right) / R}
$$

where $R=L / \pi$, and $\theta=n \pi / 2$ with $n=0,1,2,3$. The integer $n$ labels the possible consistent boundary conditions that result after imposing the folding identifications described in [10]. Notice that the KK towers contain a zero-mode only for $n=0$. The ' superscript in the summation in Eq. (2.1) indicates that the KK sums run over the restricted range $j>0, k \geq 0$ and $j=k=0$.

Given the KK wavefunctions and spectrum, we can immediately write down the expression for the propagator. It is convenient to work in configuration space in $x^{4}, x^{5}$, since this will allow us to easily project onto KK-number or momentum space as needed. However, we do work in momentum space for the four non-compact dimensions from the beginning, i.e. we use a mixed position and momentum space representation. The general representation of the scalar propagator in the compactified theory is

$$
\begin{aligned}
G_{n}\left(p ; z ; z^{\prime}\right) & =\int d^{4} x e^{i p x}\left\langle\Phi_{n}(x ; z) \Phi_{n}^{\dagger}\left(0 ; z^{\prime}\right)\right\rangle \\
& =\frac{1}{L^{2}} \sum_{j, k}^{\prime} g_{S}^{j, k} f_{n}^{(j, k)}(z)\left[f_{n}^{(j, k)}\left(z^{\prime}\right)\right]^{*}
\end{aligned}
$$


where $g_{S}^{j, k}$ is the 4-dimensional scalar propagator (in the KK-number representation)

$$
g_{S}^{j, k}=\frac{i}{p^{2}-M_{j, k}^{2}}
$$

and in the present case the spectrum is given by

$$
M_{j, k}^{2}=\frac{j^{2}+k^{2}}{R^{2}}
$$

In fact, this propagator satisfies

$$
\left(p^{2}+\partial_{4}^{2}+\partial_{5}^{2}\right) G_{n}\left(p ; z ; z^{\prime}\right)=i \delta^{(2)}\left(z-z^{\prime}\right)
$$

since

$$
\left(\partial_{4}^{2}+\partial_{5}^{2}+M_{j, k}^{2}\right) f_{n}^{(j, k)}(z)=0
$$

and the $f_{n}^{(j, k)}(z)$ form a complete set for functions satisfying the appropriate boundary conditions: ${ }^{5}$

$$
\frac{1}{L^{2}} \sum_{j, k}^{\prime} f_{n}^{(j, k)}(z)\left[f_{n}^{(j, k)}\left(z^{\prime}\right)\right]^{*}=\delta^{(2)}\left(z-z^{\prime}\right) .
$$

It is also worth keeping in mind the orthonormality relations

$$
\frac{1}{L^{2}} \int_{0}^{L} d^{2} z f_{n}^{(j, k)}(z)\left[f_{n}^{\left(j^{\prime}, k^{\prime}\right)}(z)\right]^{*}=\delta_{j, j^{\prime}} \delta_{k, k^{\prime}}
$$

which ensure the canonical normalization of the KK fields, $\phi^{(j, k)}\left(x^{\mu}\right)$.

\subsection{Propagators in Momentum Space}

Now suppose we want to work in momentum space, as opposed to KK-number space, in the compactified dimensions: $\left(p^{4}, p^{5}\right)=(m / R, l / R)$, where $m$ and $l$ are arbitrary integers, i.e. we define

$$
G_{p, n}^{\left(m, l ; m^{\prime}, l^{\prime}\right)} \equiv\left(\frac{1}{2 L}\right)^{2} \int_{-L}^{L} d^{2} z d^{2} z^{\prime} e^{i\left(p_{4} x^{4}+p_{5} x^{5}\right)} e^{-i\left(p_{4}^{\prime} x^{\prime 4}+p_{5}^{\prime} x^{\prime 5}\right)} G_{n}\left(p ; z ; z^{\prime}\right)
$$

Note that we are letting the integration run over the extended range $-L \leq x^{4}, x^{5} \leq L$; it is understood that $G_{n}\left(p ; z ; z^{\prime}\right)$ has been analytically continued outside the fundamental region $0 \leq x^{4}, x^{5} \leq L$. The factor of $(1 / 2 L)^{2}$ was introduced so that $G_{p, n}^{\left(m, l ; m^{\prime}, l^{\prime}\right)}$ has mass dimension -2 , as in four dimensions.

\footnotetext{
${ }^{5}$ If one wants to interpret Eq. (2.9) outside the fundamental region $0 \leq x^{4}, x^{5} \leq L$, the $\delta$-function on its r.h.s. should be extended in a manner consistent with the relevant boundary conditions, e.g. $\delta^{(2)}\left(\mathcal{R}(z)-z^{\prime}\right)=$ $e^{-i n \pi / 2} \delta^{(2)}\left(z-z^{\prime}\right), \delta^{(2)}\left(z-\mathcal{R}\left(z^{\prime}\right)\right)=e^{i n \pi / 2} \delta^{(2)}\left(z-z^{\prime}\right)$ and $\delta^{(2)}\left(\mathcal{R}(z)-\mathcal{R}\left(z^{\prime}\right)\right)=\delta^{(2)}\left(z-z^{\prime}\right)$, where $\mathcal{R}$ stands for a counterclockwise rotation by $\pi / 2$ in the $z$-plane. It should also be extended periodically, with period $2 L$ along both $x^{4}$ and $x^{5}$, outside $-L \leq x^{4}, x^{5} \leq L$.
} 
By using the representation of the propagator given in Eq. (2.4) we automatically obtain from Eq. (2.11) the momentum space representation satisfying the appropriate boundary conditions. In switching from KK-number to momentum space one encounters the integrals

$$
\frac{1}{4 L^{2}} \int_{-L}^{L} d^{2} z\left[h^{(m, l)}(z)\right]^{*} f_{n}^{(j, k)}(z)=\frac{1}{2\left[1+\delta_{j, 0} \delta_{k, 0}\right]} \hat{\delta}(m, l ; j, k ; n),
$$

where we used the explicit form of the KK wavefunctions given in Eq. (2.2), as well as the orthonormality relations

$$
\frac{1}{4 L^{2}} \int_{-L}^{L} d^{2} z h^{(m, l)}(z)\left[h^{\left(m^{\prime}, l^{\prime}\right)}(z)\right]^{*}=\delta_{m, m^{\prime}} \delta_{l, l^{\prime}}
$$

that hold for the standard plane waves given in Eq. (2.3). We also defined a "generalized" Kronecker delta

$$
\hat{\delta}\left(m, l ; m^{\prime}, l^{\prime} ; n\right)=\delta_{m, m^{\prime}} \delta_{l, l^{\prime}}+e^{i \theta} \delta_{m, l^{\prime}} \delta_{l,-m^{\prime}}+e^{2 i \theta} \delta_{m,-m^{\prime}} \delta_{l,-l^{\prime}}+e^{3 i \theta} \delta_{m,-l^{\prime}} \delta_{l, m^{\prime}},
$$

where $\theta=n \pi / 2$. Note that when the quantum numbers $m, l, m^{\prime}, l^{\prime}$ are all taken positive, Eq. (2.14) coincides with the standard two-dimensional Kronecker delta. The additional terms take into account the boundary conditions and depend on the integer $n$. However, note also that for the case of the zero-mode (which only arises for $n=0$ ) one has $\hat{\delta}(m, l ; 0,0 ; n=0)=4 \delta_{m, 0} \delta_{l, 0}$, with an extra factor of 4 .

\subsubsection{Diagonal Propagators}

For a propagator with the general representation in KK-number space,

$$
G_{n}\left(p ; z ; z^{\prime}\right)=\frac{1}{L^{2}} \sum_{j, k}^{\prime} g_{j, k} f_{n}^{(j, k)}(z)\left[f_{n}^{(j, k)}\left(z^{\prime}\right)\right]^{*}
$$

we can also write

$$
G_{n}\left(p ; z ; z^{\prime}\right)=\frac{1}{4 L^{2}} \sum_{m, l} \sum_{m^{\prime}, l^{\prime}} G_{p, n}^{\left(m, l ; m^{\prime}, l^{\prime}\right)} h^{(m, l)}(z)\left[h^{\left(m^{\prime}, l^{\prime}\right)}\left(z^{\prime}\right)\right]^{*},
$$

with $G_{p, n}^{\left(m, l ; m^{\prime}, l^{\prime}\right)}$ as defined in Eq. 2.11). Notice that the sums in Eq. (2.16) run over all integers. We will reserve the labels $m$ and $l$ to denote the momentum along the compact dimensions in units of $1 / R$, hence running unrestricted over integer values, while leaving the labels $j$ and $k$ to denote the KK numbers, which can take integer values on the restricted range $j>0, k \geq 0$ and $j=k=0$. For a scalar field one finds the simple result, derived in Appendix A,

$$
G_{p, n}^{\left(m, l ; m^{\prime}, l^{\prime}\right)}=\frac{i}{p^{2}-M_{m, l}^{2}} \hat{\delta}\left(m, l ; m^{\prime}, l^{\prime} ; n\right) .
$$

Therefore, the information about the boundary conditions is contained in the "generalized" $\delta$ functions defined in Eq. (2.14). Note that for the zero mode, $G_{p, n=0}^{(m, l ; 0,0)}=4\left(i / p^{2}\right) \delta_{m, 0} \delta_{l, 0}$, one finds an additional factor of 4 in the momentum representation, compared to the zero-mode propagator $g_{S}^{0,0}=i / p^{2}$ in the KK-number representation. 


\subsubsection{Kaluza-Klein Mixing}

It is also useful to derive the relation between the KK-number and momentum space representations of the propagator when transitions among different KK states are allowed. This situation arises when interactions are included and the unperturbed KK states defined in Eqs. (2.1) and (2.2) are not exact mass eigenstates. These KK-number violating transitions are of great phenomenological interest and we will consider how they arise in greater detail in later sections.

Here we simply note that quite generally we can represent the full propagator either in the KK-number basis or the momentum basis, along the lines discussed previously:

$$
\begin{aligned}
G\left(p ; z ; z^{\prime}\right) & =\frac{1}{L^{2}} \sum_{j, k}^{\prime} \sum_{j^{\prime}, k^{\prime}}^{\prime} g_{(j, k) ;\left(j^{\prime}, k^{\prime}\right)} f_{n}^{(j, k)}(z)\left[f_{n}^{\left(j^{\prime}, k^{\prime}\right)}\left(z^{\prime}\right)\right]^{*} \\
& =\frac{1}{4 L^{2}} \sum_{m, l} \sum_{m^{\prime}, l^{\prime}} G_{n}^{\left(m, l ; m^{\prime}, l^{\prime}\right)} h^{(m, l)}(z)\left[h^{\left(m^{\prime}, l^{\prime}\right)}\left(z^{\prime}\right)\right]^{*},
\end{aligned}
$$

the only new ingredient being the possibility of non-diagonal KK transitions. In Appendix A we derive the general relation between the expansion coefficients $G_{n}^{\left(m, l ; m^{\prime}, l^{\prime}\right)}$ and $g_{(j, k) ;\left(j^{\prime}, k^{\prime}\right)}$. The specific form of the momentum space coefficients $G_{n}^{\left(m, l ; m^{\prime}, l^{\prime}\right)}$ encodes the appropriate boundary conditions.

A special case of interest arises when the momentum quantum numbers $m$ and $l$ take on positive values:

$$
g_{(j, k) ;\left(j^{\prime}, k^{\prime}\right)}=G_{n}^{\left(j, k ; j^{\prime}, k^{\prime}\right)} \quad \text { for } j, j^{\prime}>0 \text { and } k, k^{\prime} \geq 0 .
$$

Also, when a zero mode is involved we get:

$$
\begin{array}{rlrl}
g_{(j, k) ;(0,0)} & =\frac{1}{2} G_{n}^{(j, k ; 0,0)} & & \text { for } j>0, k \geq 0 \\
g_{(0,0) ;\left(j^{\prime}, k^{\prime}\right)} & =\frac{1}{2} G_{n}^{\left(0,0 ; j^{\prime}, k^{\prime}\right)} & & \text { for } j^{\prime}>0, k^{\prime} \geq 0 \\
g_{(0,0) ;(0,0)} & =\frac{1}{4} G_{n}^{(0,0 ; 0,0)} . &
\end{array}
$$

Note that the factor of 4 relating $g_{(0,0) ;(0,0)}$ and $G_{n}^{(0,0 ; 0,0)}$ is similar to the one found in the diagonal case studied in subsection 2.2.1.

Relations (2.19) and (2.20) are useful to obtain the KK-number expansion coefficients, which contain all physical information, from the momentum space expansion coefficients, which are more easily calculated in certain situations.

\subsection{KK-Number violating Structure due to Localized Operators}

As mentioned in the Introduction, the chiral square compactification has conical singularities at the corners of the fundamental square region, with coordinates $\left(x^{4}, x^{5}\right)=(0,0),(L, L)$ and $(0, L)$. In this section we consider the effect of operators localized at these special points. In fact, the calculation of loops involving bulk interactions reveals logarithmic divergences that require counterterms localized precisely at these points. In later sections we shall show by 
explicit computation that the divergences at one-loop order have precisely this property. It will be useful to define the shorthand notation

$$
\delta_{c}(z) \equiv \delta\left(x^{4}\right) \delta\left(x^{5}\right)+\delta\left(L-x^{4}\right) \delta\left(L-x^{5}\right)+c \delta\left(x^{4}\right) \delta\left(L-x^{5}\right),
$$

where $c$ is a dimensionless coupling that parametrizes the strength of the operators at $(0, L)$ relative to those at $(0,0)$ and $(L, L)$. We assume that operators localized at $(0,0)$ and $(L, L)$ appear with identical coefficients, as required by KK-parity.

Let us consider the most general set of localized kinetic terms for a complex scalar field $\Phi$

$$
\begin{aligned}
& \frac{1}{4} \delta_{c_{1}}(z) \times r_{1} \partial_{\mu} \Phi^{\dagger} \partial^{\mu} \Phi+\left[\frac{1}{4} \delta_{c_{2}}(z) \times r_{2} \Phi^{\dagger}\left(\partial_{+} \partial_{-} \Phi\right)+\text { h.c. }\right] \\
& -\frac{1}{4} \delta_{c_{3}}(z) \times r_{3}\left(\partial_{+} \Phi^{\dagger}\right)\left(\partial_{-} \Phi\right)-\frac{1}{4} \delta_{c_{3}^{\prime}}(z) \times r_{3}^{\prime}\left(\partial_{-} \Phi^{\dagger}\right)\left(\partial_{+} \Phi\right)
\end{aligned}
$$

where

$$
\partial_{ \pm}=\partial_{4} \pm i \partial_{5}
$$

The constants $r_{i}, c_{i}$ for $i=1,2,3$ and $r_{3}^{\prime}, c_{3}^{\prime}$ are arbitrary. For convenience, we extracted a factor of $(1 / 2)^{2}$ to account for an enhancement due to the KK wavefunctions in Eq. (2.2), evaluated at the singular points. Note that the $c_{i}$ are dimensionless, but the $r_{i}$ have dimensions of length squared. Notice also that the coefficients of the 4 D-like kinetic term need not be the same as for the kinetic operators with derivatives in the compact directions, $\partial_{ \pm}$. However, we assumed a rotational symmetry in the transverse $x^{4}-x^{5}$ plane that forces the $\partial_{4}$ and $\partial_{5}$ derivatives to appear on an equal footing. This is natural given the rotational symmetry of the conical singularities, and will be explicitly checked by the one-loop computation in the following sections.

Using the scalar propagator representation given in Eq. (2.4), the contribution of the first term in Eq. (2.22) to the two-point function is

$$
\begin{aligned}
\underset{z}{\longrightarrow} \stackrel{y}{\longrightarrow \longrightarrow} \longrightarrow & =\int_{0}^{L} d^{2} y G\left(p ; z^{\prime}, y\right)\left[\frac{1}{4} \delta_{c_{1}}(y) i r_{1} p^{2}\right] G(p ; y, z) \\
& =\left(\frac{1}{L^{2}}\right)^{2} \sum_{j, k}^{\prime} \sum_{j^{\prime}, k^{\prime}}^{\prime} g_{S}^{j, k} f_{n}^{(j, k)}\left(z^{\prime}\right)\left[i r_{1} p^{2} K_{c_{1}}^{(j, k)\left(j^{\prime}, k^{\prime}\right)}\right] g_{S}^{j^{\prime}, k^{\prime}}\left[f_{n}^{\left(j^{\prime}, k^{\prime}\right)}(z)\right]^{*},
\end{aligned}
$$

where the cross represents an insertion of the localized 4D-like kinetic term in Eq. (2.22), and

$$
\begin{aligned}
K_{c}^{(j, k)\left(j^{\prime}, k^{\prime}\right)}= & \frac{1}{4}\left\{\left[f_{n}^{(j, k)}(0,0)\right]^{*} f_{n}^{\left(j^{\prime}, k^{\prime}\right)}(0,0)+\left[f_{n}^{(j, k)}(L, L)\right]^{*} f_{n}^{\left(j^{\prime}, k^{\prime}\right)}(L, L)\right. \\
& \left.+c\left[f_{n}^{(j, k)}(0, L)\right]^{*} f_{n}^{\left(j^{\prime}, k^{\prime}\right)}(0, L)\right\},
\end{aligned}
$$

with the KK wavefunctions $f_{n}^{(j, k)}$ defined in Eq. (2.2). Insertions of the kinetic terms involving derivatives in the extra dimensions can be treated along the same lines with the help of the relations

$$
\partial_{ \pm} f_{n}^{(j, k)}(z)=i r_{j, \pm k} M_{j, k} f_{n \mp 1}^{(j, k)}(z)
$$


where the $r_{j, k}$ are complex phases defined by

$$
r_{j, k} \equiv \frac{j+i k}{\sqrt{j^{2}+k^{2}}}
$$

We see that Eq. (2.24) has the general KK-number mixing structure of Eq. (2.18). In order to interpret the result of the one-loop computations we present in the following sections, it is useful to consider the momentum space representation of the previous process. Using Eq. (2.19) we write the contribution to the two-point function in momentum space, $\left(p^{4}, p^{5}\right)=(m / R, l / R)$, when $m, l>0$, for the four types of boundary conditions $n=0,1,2$ or 3 .

Consider first scalar fields satisfying $n=0$ boundary conditions, i.e. having a zero-mode in its KK spectrum. Assuming for simplicity that $r_{2}, c_{2}$ are real, we get

$$
\begin{aligned}
G_{n}^{\left(m, l ; m^{\prime}, l^{\prime}\right)} & =\stackrel{m^{\prime}, l^{\prime}}{\longrightarrow} \longrightarrow \underset{\bullet}{\boldsymbol{\otimes}} \stackrel{m, l}{\bullet} \\
& =g_{S}^{m, l}\left\{i \frac{r_{1}}{L^{2}} p^{2} K_{c_{1}}^{(m, l)\left(m^{\prime}, l^{\prime}\right)}-i \frac{r_{2}}{L^{2}}\left(M_{m, l}^{2}+M_{m^{\prime}, l^{\prime}}^{2}\right) K_{c_{2}}^{(m, l)\left(m^{\prime}, l^{\prime}\right)}\right\} g_{S}^{m^{\prime}, l^{\prime}},
\end{aligned}
$$

where, for these boundary conditions, Eqs. (2.2) and (2.25) give

$$
K_{c}^{(m, l)\left(m^{\prime}, l^{\prime}\right)}=\frac{4\left[1+(-1)^{m+l+m^{\prime}+l^{\prime}}\right]+c\left[(-1)^{m}+(-1)^{l}\right]\left[(-1)^{m^{\prime}}+(-1)^{l^{\prime}}\right]}{4\left(1+\delta_{m, 0} \delta_{l, 0}\right)\left(1+\delta_{m^{\prime}, 0} \delta_{l^{\prime}, 0}\right)} .
$$

It is also easy to see that the operators proportional to $r_{3}$ and $r_{3}^{\prime}$ in Eq. (2.22) vanish in this case.

Note that, as a result of the operators at $(0,0)$ and $(L, L)$ being equal, the induced KK transitions are non-vanishing only when $m+l$ and $m^{\prime}+l^{\prime}$ are both even or both odd, thus preserving precisely the KK parity defined by $(-1)^{m+l}$. Furthermore, it is useful to notice that Eq. (2.29) gives rise to precisely three different non-vanishing cases, which we find convenient to order as follows

Case 1a: $\quad(-1)^{m+l}=(-1)^{m^{\prime}+l^{\prime}}=+1, \quad m-m^{\prime}$ even,

Case 1b: $\quad(-1)^{m+l}=(-1)^{m^{\prime}+l^{\prime}}=+1, \quad m-m^{\prime}$ odd,

Case 2: $\quad(-1)^{m+l}=(-1)^{m^{\prime}+l^{\prime}}=-1$.

That is, localized operators distinguish between KK-number transitions among KK-parity even states and KK-parity odd states. Notice that the distinction arises from operators localized at $(0, L)$. Furthermore, for KK-parity even transitions, there is a difference depending on whether $m-m^{\prime}$ is even or odd.

For $n=2$ boundary conditions, the momentum space two-point function with insertions of the localized operators in Eq. (2.22) has the structure of Eq. (2.28) with

$$
K_{c}^{(m, l)\left(m^{\prime}, l^{\prime}\right)}=\frac{c\left[(-1)^{m}-(-1)^{l}\right]\left[(-1)^{m^{\prime}}-(-1)^{l^{\prime}}\right]}{4\left(1+\delta_{m, 0} \delta_{l, 0}\right)\left(1+\delta_{m^{\prime}, 0} \delta_{l^{\prime}, 0}\right)} .
$$


In this situation, we obtain three cases that we label as follows

$$
\begin{aligned}
& \text { Case 1: } \quad(-1)^{m+l}=(-1)^{m^{\prime}+l^{\prime}}=+1, \\
& \text { Case 2a: } \quad(-1)^{m+l}=(-1)^{m^{\prime}+l^{\prime}}=-1, \quad m-m^{\prime} \text { even, } \\
& \text { Case 2b: } \quad(-1)^{m+l}=(-1)^{m^{\prime}+l^{\prime}}=-1, \quad m-m^{\prime} \text { odd. }
\end{aligned}
$$

Note that for $n=2$, it is the transitions between KK-parity odd states that contain two subcases, $2 \mathrm{a}$ and $2 \mathrm{~b}$, while for $n=0$, the subcases appeared when KK-parity even transitions are considered, $1 \mathrm{a}$ and $1 \mathrm{~b}$.

Finally, for $n=1$ or $n=3$ boundary conditions, the localized 4D-like kinetic term does not contribute to the two-point function as a result of the vanishing of the KK wavefunctions at the three conical singularities. Only the operators proportional to $r_{3}$ and $r_{3}^{\prime}$ in Eq. (2.22) give a nonvanishing contribution:

$$
\begin{aligned}
G_{n=1,3}^{\left(m, l ; m^{\prime}, l^{\prime}\right)}=g_{S}^{m, l}\left\{-i \frac{r_{3}}{L^{2}}\right. & r_{m, l} r_{m^{\prime}, l^{\prime}}^{*} M_{m, l} M_{m^{\prime}, l^{\prime}} K_{c_{3}}^{(m, l)\left(m^{\prime}, l^{\prime}\right)} \\
& \left.-i \frac{r_{3}^{\prime}}{L^{2}} r_{m, l}^{*} r_{m^{\prime}, l^{\prime}} M_{m, l} M_{m^{\prime}, l^{\prime}} K_{c_{3}^{\prime}}^{(m, l)\left(m^{\prime}, l^{\prime}\right)}\right\} g_{S}^{m^{\prime}, l^{\prime}}
\end{aligned}
$$

To obtain this, we used Eq. (2.26) as well as the fact that $\left[f_{1}^{(j, k)}(z)\right]^{*}=-f_{3}^{(j, k)}(z)$, from Eq. (2.2). When $n=3, K_{c_{3}}^{(m, l)\left(m^{\prime}, l^{\prime}\right)}$ is as given in Eq. (2.29) and $K_{c_{3}^{\prime}}^{(m, l)\left(m^{\prime}, l^{\prime}\right)}$ as in Eq. (2.31), while for $n=1$, it is the other way around. Notice that for $n=1$ or 3 one generically gets four different non-vanishing results, corresponding to cases 1a, 1b, 2a and 2b in Eqs. (2.30) and (2.32).

By checking that loop contributions to the two point function have the structure of Eqs. 2.28) and (2.33) we shall be able to confirm in later sections that they correspond to localized operators as in Eq. (2.22).

\section{Chiral Fermions}

Having discussed various general properties in the scalar case, and before we can tackle the calculation of one-loop radiative corrections in this class of theories, we need to consider the form of the propagator in momentum space for fields transforming non-trivially under the 6D Lorentz group. In this section, we treat the case of $6 \mathrm{D}$ chiral fermions, $\Psi_{ \pm}$, where + or - label the $6 \mathrm{D}$ chirality, according to the projection operators $P_{ \pm}=\frac{1}{2}(1 \pm \bar{\Gamma})$, with $\bar{\Gamma}$ the $6 \mathrm{D}$ chirality operator. In the following section we consider the propagators associated with $6 \mathrm{D}$ gauge fields, which contain both spin-1 and spin-0 components under the unbroken 4D Lorentz group.

As shown in [10], fermions propagating in six dimensions with the two extra dimensions compactified on the chiral square have a chiral zero-mode. Starting from the free fermion action ( $\Gamma^{M}$ are $8 \times 8$ Dirac $\Gamma$-matrices, and $M=0,1, \ldots, 5$ runs over the $6 \mathrm{D}$ Lorentz indices)

$$
S_{\Psi}=\int d^{4} x \int_{0}^{L} d x^{4} \int_{0}^{L} d x^{5} \frac{i}{2}\left[\bar{\Psi} \Gamma^{M} \partial_{M} P_{ \pm} \Psi-\left(\partial_{M} \bar{\Psi}\right) \Gamma^{M} P_{ \pm} \Psi\right]
$$


and imposing the identification of adjacent sides of the square region $0<x^{4}, x^{5}<L$, one can show that the two 4-dimensional chiralities, $\Psi_{ \pm L}$ and $\Psi_{ \pm R}$, contained in $\Psi_{ \pm} \equiv P_{ \pm} \Psi$, satisfy boundary conditions determined by integers $n_{L}^{ \pm}$and $n_{R}^{ \pm}$such that

$$
n_{R}^{ \pm}=n_{L}^{ \pm} \mp 1 \bmod 4
$$

This shows that only a chiral zero-mode is allowed. The propagator associated with this system is obtained by inverting the operator appearing in Eq. (3.1), taking care of imposing the appropriate boundary conditions. We leave the details to Appendix B. The resulting propagator in the momentum space representation takes the form

$$
G_{p}^{ \pm,\left(m, l ; m^{\prime}, l^{\prime}\right)}=P_{ \pm} \Gamma^{M} p_{M} g_{S}^{m, l}\left[P_{R} \hat{\delta}\left(m, l ; m^{\prime}, l^{\prime} ; n_{L}^{ \pm}\right)+P_{L} \hat{\delta}\left(m, l ; m^{\prime}, l^{\prime} ; n_{R}^{ \pm}\right)\right],
$$

where $p_{M}=\left(p_{\mu}, p_{4}, p_{5}\right)$ with $p_{4}=-m / R$ and $p_{5}=-l / R$ [the minus signs arising from the Minkowski metric], and $n_{L}^{ \pm}, n_{R}^{ \pm}$are related by Eq. (3.2). $g_{S}^{m, l}$ is the scalar propagator given in Eq. (2.5). When using Eq. (3.3), one should be careful to remember that the $4 \mathrm{D}$ chirality projectors, $P_{L, R}$, distinguish between the $\Gamma^{\mu}$ and $\Gamma^{4,5}$ terms in $\Gamma^{M} p_{M}$. In particular, $P_{L, R}$ commute with $\Gamma^{4}$ and $\Gamma^{5}$.

\section{Gauge fields}

We turn now our attention to the propagators associated with the $6 \mathrm{D}$ gauge system. We start from the action

$$
S=\int d^{4} x \int_{0}^{L} d x^{4} \int_{0}^{L} d x^{5}\left(-\frac{1}{4} F_{M N} F^{M N}+\mathcal{L}_{G F}\right)
$$

where the indices $M, N$ run over the six spacetime coordinates. After compactification, the components of the $6 \mathrm{D}$ gauge field naturally separate into $A_{\mu}(\mu=0,1,2,3)$ and $A_{4}, A_{5}$. While the former are part of a spin-1 field, the latter constitute two scalar degrees of freedom from a four-dimensional point of view.

A convenient choice of gauge arises by requiring that the mixings of $A_{\mu}$ with $A_{4}$ and $A_{5}$ vanish:

$$
\mathcal{L}_{G F}=-\frac{1}{2 \xi}\left[\partial_{\mu} A^{\mu}-\xi\left(\partial_{4} A_{4}+\partial_{5} A_{5}\right)\right]^{2},
$$

where $\xi$ is a gauge fixing parameter. This gauge clarifies how the physical degrees of freedom are encoded in the $6 \mathrm{D}$ field, $A_{M}$ : at each massive KK level, one linear combination of the two scalars, $A_{4}$ and $A_{5}$ is eaten by the massive $A_{\mu}$ fields, while the orthogonal combination remains as an additional scalar degree of freedom. This system was studied in detail in [11, where the appropriate boundary conditions and resulting interactions were worked out. We assume that $A_{\mu}$ satisfies boundary conditions corresponding to $n=0$, so that the spin- $1 \mathrm{KK}$ towers have a zero-mode. In other words, we do not consider the case where the gauge symmetry is broken by the boundary conditions. One also finds that the linear combinations

$$
A_{ \pm}=A^{4} \pm i A^{5}
$$


satisfy boundary conditions give by $n=3$ for $A_{+}$and $n=1$ for $A_{-}$. In particular, there are no zero-modes in the scalar sector. We refer to these scalars as "spinless adjoints".

In the following subsections we derive the propagators for the spin- 1 and spin- 0 components, as well as the ghost fields associated with the gauge fixing term Eq. (4.2).

\subsection{The Spin-1 Components}

Apart from the boundary conditions, which are treated as for the scalar case in section 2, the derivation of the spin-1 propagator in momentum space is identical to the $4 \mathrm{D}$ derivation. We obtain

$$
G_{\mu \nu, p}^{\left(m, l ; m^{\prime}, l^{\prime}\right)}=g_{\mu \nu}^{m, l} \hat{\delta}\left(m, l ; m^{\prime}, l^{\prime} ; 0\right)
$$

where

$$
g_{\mu \nu}^{m, l}=-\left[\eta_{\mu \nu}-(1-\xi) \frac{p_{\mu} p_{\nu}}{p^{2}-\xi M_{m, l}^{2}}\right] g_{S}^{m, l},
$$

and $g_{S}^{m, l}$ is given in Eq. (2.5). We recognize the 4-dimensional propagators appropriate to the gauge fixing term (4.2) as those for a (massive) gauge field in an $R_{\xi}$ gauge.

\subsection{The Spin-0 Components}

As shown in Appendix $\mathbb{B}$, the momentum space propagator, defined as the inverse of the quadratic operator associated with the $A_{4}-A_{5}$ system in the free Lagrangian, can be more easily derived in the $A_{ \pm}$basis, defined by Eq. (4.3), where the boundary conditions are well defined. The result is a $2 \times 2$ matrix with components

$$
\begin{aligned}
& \left(\begin{array}{cc}
G_{p,++}^{\left(m, l ; m^{\prime}, l^{\prime}\right)} & G_{p,+-}^{\left(m, l ; m^{\prime}, l^{\prime}\right)} \\
G_{p,-+}^{\left(m, l ; m^{\prime}, l^{\prime}\right)} & G_{p,--}^{\left(m, l ; m^{\prime}, l^{\prime}\right)}
\end{array}\right)= \\
& \left(\begin{array}{cc}
\left(g_{h}^{m, l}+g_{\phi}^{m, l}\right) \hat{\delta}\left(m, l ; m^{\prime}, l^{\prime} ; 3\right) & -r_{m, l}^{2}\left(g_{h}^{m, l}-g_{\phi}^{m, l}\right) \hat{\delta}\left(m, l ; m^{\prime}, l^{\prime} ; 1\right) \\
-r_{m, l}^{* 2}\left(g_{h}^{m, l}-g_{\phi}^{m, l}\right) \hat{\delta}\left(m, l ; m^{\prime}, l^{\prime} ; 3\right) & \left(g_{h}^{m, l}+g_{\phi}^{m, l}\right) \hat{\delta}\left(m, l ; m^{\prime}, l^{\prime} ; 1\right)
\end{array}\right),
\end{aligned}
$$

where

$$
g_{h}^{m, l}=\frac{i}{p^{2}-M_{m, l}^{2}}, \quad g_{\phi}^{m, l}=\frac{i}{p^{2}-\xi M_{m, l}^{2}},
$$

and the complex phases $r_{m, l}$ were defined in Eq. (2.27). The pole structure shown in Eq. (4.7) reveals that the $A_{4}-A_{5}$ system has two degrees of freedom at each KK level, one with mass $M_{m, l}$ and the second with mass $\sqrt{\xi} M_{m, l}$. The scalar mode with the $\xi$-dependent mass corresponds to the longitudinal degree of freedom of the massive spin- 1 gauge fields, as required by the higher dimensional Higgs mechanism.

Taking into account the fact that $A_{-}^{\dagger}=A_{+}$, one can show that the relation between the various components $G_{++}, G_{+-}, G_{-+}$and $G_{--}$in Eq. (4.6), and the tree-level two-point functions 
that enter the Feynman rules is given by

$$
\begin{aligned}
\left\langle A_{+}^{m, l} A_{+}^{m^{\prime}, l^{\prime} \dagger}\right\rangle & =\frac{1}{2}\left[G_{p,++}^{\left(m, l ; m^{\prime}, l^{\prime}\right)}+G_{p,--}^{\left(-m^{\prime},-l^{\prime} ;-m,-l\right)}\right]=G_{p,++}^{\left(m, l ; m^{\prime}, l^{\prime}\right)} \\
\left\langle A_{+}^{m, l} A_{+}^{m^{\prime}, l^{\prime}}\right\rangle & =\frac{1}{2}\left[G_{p,+-}^{\left(m, l ; m^{\prime}, l^{\prime}\right)}+G_{p,+-}^{\left(-m^{\prime},-l^{\prime} ;-m,-l\right)}\right]=G_{p,+-}^{\left(m, l ; m^{\prime}, l^{\prime}\right)}
\end{aligned}
$$

together with their complex conjugates, were we chose to express all correlators in terms of $A_{+}$ and $A_{+}^{\dagger}$. The second equalities follow from the explicit solution for $G^{\left(m, l ; m^{\prime}, l^{\prime}\right)}$ given in Eq. (4.6).

\subsection{Faddeev-Popov Ghosts}

The ghost Lagrangian associated with the gauge fixing term, Eq. (4.2), is

$$
-\bar{c}^{a}\left[\partial_{\mu} D^{\mu}-\xi\left(\partial_{4} D_{4}+\partial_{5} D_{5}\right)\right] c^{a}
$$

where the ghost fields, $c^{a}$, satisfy the same boundary conditions as $A_{\mu}$, i.e. given by $n=0$. By comparing to the derivation of the scalar propagator, Eq. (2.17), it is easy to see that the ghost propagator in momentum space is given by

$$
G_{\xi, p}^{\left(m, l ; m^{\prime}, l^{\prime}\right)}=\frac{i}{p^{2}-\xi M_{m, l}^{2}} \hat{\delta}\left(m, l ; m^{\prime}, l^{\prime} ; 0\right),
$$

i.e., it has a $\xi$-dependent mass given by $\sqrt{\xi} M_{m, l}$.

\section{Radiative Corrections}

Having at our disposal the propagators for 6D scalars, Weyl fermions and gauge fields that correctly encode the boundary conditions appropriate in the chiral square background, we are in a position to consider the one-loop structure of the theory. In this section we compute the quantum corrections to the two-point functions (sometimes we will refer to these as self-energies, even though they also include mixing among KK states) for gauge, fermion and scalar fields and show that, besides a renormalization of the bulk kinetic terms, the two point correlation functions contain logarithmic divergences corresponding to counterterms localized at the conical singularities with coordinates $\left(x^{4}, x^{5}\right)=(0,0),(L, L)$ and $(0, L)$.

\subsection{Gauge Boson Two-Point Function}

We first compute the one loop contributions to the gauge boson two-point function. We consider in turn scalar matter, fermionic matter and the gauge self-interactions themselves. We present the scalar case in considerable detail to show how the KK-number violating structure corresponding to localized operators arises. The lessons thus learned carry straightforwardly to the remaining types of one-loop graphs. 


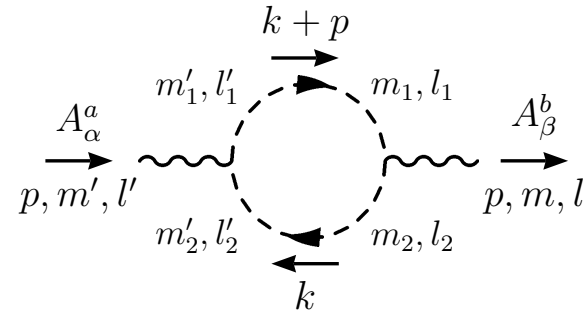

(a)

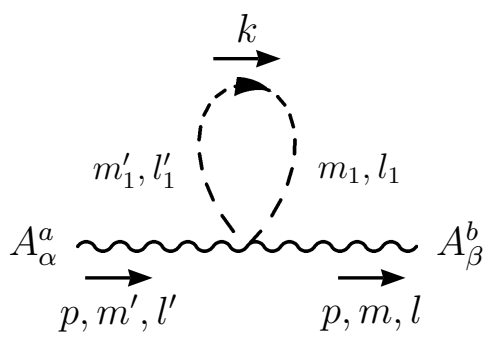

(b)

Figure 1: One-loop contributions to the gauge boson self energy due to a minimally coupled scalar. The external four-dimensional momentum is denoted by $p$. The momenta along the extra dimensions are simply denoted by the corresponding integer quantum number according to $p_{4}=m / R, p_{5}=l / R$, etc. Since the momentum in the compact dimensions is not conserved, each line is labeled by two sets of momenta.

\subsubsection{Scalar Matter}

We start by evaluating the one-loop diagrams arising from scalars minimally coupled to gauge bosons. We consider 6D scalar fields satisfying any of the possible boundary conditions, labeled by $n=0,1,2$ or 3 . We evaluate these diagrams in detail to show how the operators localized at the three conical singularities, with coordinates $\left(x^{4}, x^{5}\right)=(0,0),(0, L)$ and $(L, L)$, arise. We need only consider the divergent pieces. For fixed KK numbers of the external lines, $(j, k)$ and $\left(j^{\prime}, k^{\prime}\right)$, these split into two different categories:

- Terms with a KK-number structure identical to the tree-level propagator, Eq. (4.4), i.e. proportional to $\hat{\delta}\left(j, k ; j^{\prime}, k^{\prime} ; 0\right)$. After KK summation, the result grows as a power of the number of KK modes. This power-law divergence renormalizes 6D bulk operators, and is of no interest to us here.

- Terms with a KK-number structure different from the tree-level propagator (i.e. KKnumber violating). At one loop, only a finite number of KK states contribute, and the divergences are only logarithmic, as in a $4 \mathrm{D}$ computation. We would like to see that the KK-number violating structure that arises here corresponds precisely to the one induced by operators localized at $(0,0),(0, L)$ and $(L, L)$.

In order to see the above features it is useful to concentrate on the two-point function, as opposed to the amputated diagrams. Let us start with the diagram involving the interaction between two scalars and two gauge bosons, as in Figure 1 (b). In momentum space, we have

$$
\left\langle A_{\mu}^{(j, k)} A_{\nu}^{\left(j^{\prime}, k^{\prime}\right)}\right\rangle^{(b)}=\left(\frac{1}{4 L^{2}}\right)^{2}\left(\frac{1}{4}\right) \sum_{m, l} \sum_{m^{\prime}, l^{\prime}} G_{\mu, p}^{\alpha(j, k ; m, l)}\left[\left\langle A_{\alpha}^{(m, l)} A_{\beta}^{\left(m^{\prime}, l^{\prime}\right)}\right\rangle_{\mathrm{amp}}^{(b)}\right] G_{\nu, p}^{\beta\left(m^{\prime}, l^{\prime} ; j^{\prime}, k^{\prime}\right)}
$$


where each propagator carries a factor of $1 /\left(4 L^{2}\right)$, as in Eq. (2.18), and the additional factor of $1 / 4$ arises from the integration over interaction points in spacetime, $\int_{0}^{L} d^{2} y=(1 / 4) \int_{-L}^{L} d^{2} y$. Using the Feynman rules shown in Figure 2, the amputated function in Figure 1 (b) is

$$
\left\langle A_{\alpha}^{(m, l)} A_{\beta}^{\left(m^{\prime}, l^{\prime}\right)}\right\rangle_{\mathrm{amp}}^{(b)}=2 i g_{6}^{2} \operatorname{Tr}\left(T^{a} T^{b}\right) \eta_{\alpha \beta} \sum_{m_{1}, l_{1}} \int \frac{d^{D} k}{(2 \pi)^{D}} G_{k, n}^{\left(m_{1}, l_{1} ; m_{1}^{\prime}, l_{1}^{\prime}\right)},
$$

where the scalar propagator, $G_{k, n}^{\left(m_{1}, l_{1} ; m_{1}^{\prime}, l_{1}^{\prime}\right)}$, was defined in Eq. (2.17). It is understood that, due to momentum conservation, $m_{1}^{\prime}=m_{1}+m^{\prime}-m$ and $l_{1}^{\prime}=l_{1}+l^{\prime}-l$. Notice that the factor of $1 /\left(4 L^{2}\right)$ associated with the internal propagator disappears after integrating $\int_{-L}^{L} d^{2} y$, according to the orthonormality condition, Eq. (2.13).

Consider the KK sums in the two point function (5.1). The first term in $\hat{\delta}\left(m_{1}, l_{1} ; m_{1}^{\prime}, l_{1}^{\prime} ; n\right)$ [see Eq. (2.14)] leads to

$$
\sum_{m, l} \sum_{m^{\prime}, l^{\prime}} \hat{\delta}(j, k ; m, l ; 0)\left[\delta_{m_{1}, m_{1}^{\prime}} \delta_{m_{2}, m_{2}^{\prime}}\right] \hat{\delta}\left(m^{\prime}, l^{\prime} ; j^{\prime}, k^{\prime} ; 0\right)=4 \hat{\delta}\left(j, k ; j^{\prime}, k^{\prime} ; 0\right)
$$

which has the KK-number structure of the tree-level propagator, arising from the bulk kinetic terms. Since $G_{k, n}^{\left(m_{1}, l_{1} ; m_{1}^{\prime}, l_{1}^{\prime}\right)}=i\left(k^{2}-M_{m_{1}, l_{1}}^{2}\right)^{-1} \hat{\delta}\left(m_{1}, l_{1} ; m_{1}^{\prime}, l_{1}^{\prime} ; n\right)$, the $4 \mathrm{D}$ momentum integral in Eq. (5.2) is quadratically divergent. In dimensional regularization, the divergent part is proportional to $M_{m_{1}, l_{1}}^{2} \sim m_{1}^{2}+l_{1}^{2}$, and it is clear that the contribution from Eq. (5.3) diverges like $\sum_{m_{1}, l_{1}}\left(m_{1}^{2}+l_{1}^{2}\right)$. This power-law divergence corresponds to a renormalization of the bulk gauge kinetic term.

The contribution due to the three remaining terms in $\hat{\delta}\left(m_{1}, l_{1} ; m_{1}^{\prime}, l_{1}^{\prime} ; n\right)$ lead to a KKnumber violating structure corresponding to localized operators at the three corners of the chiral square, as follows. These terms fix the loop momenta $m_{1}, l_{1}$ in terms of $m-m^{\prime}$ and $l-l^{\prime}$ according to

$$
\begin{array}{ll}
\delta_{m_{1}, l_{1}^{\prime}} \delta_{l_{1},-m_{1}^{\prime}} \rightarrow m_{1}=\frac{1}{2}\left[\left(m-m^{\prime}\right)-\left(l-l^{\prime}\right)\right], & l_{1}=\frac{1}{2}\left[\left(m-m^{\prime}\right)+\left(l-l^{\prime}\right)\right], \\
\delta_{m_{1},-m_{1}^{\prime}} \delta_{l_{1},-l_{1}^{\prime}} \rightarrow m_{1}=\frac{1}{2}\left(m-m^{\prime}\right), & l_{1}=\frac{1}{2}\left(l-l^{\prime}\right), \\
\delta_{m_{1},-l_{1}^{\prime}} \delta_{l_{1}, m_{1}^{\prime}} \rightarrow m_{1}=\frac{1}{2}\left[\left(m-m^{\prime}\right)+\left(l-l^{\prime}\right)\right], & l_{1}=\frac{1}{2}\left[\left(m-m^{\prime}\right)-\left(l-l^{\prime}\right)\right] .
\end{array}
$$
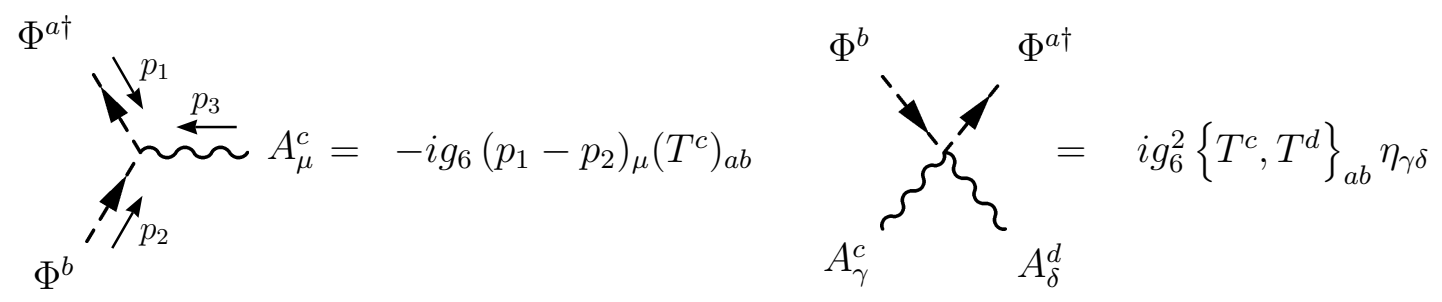

Figure 2: Momentum space Feynman rules for scalars minimally coupled to gauge bosons in the representation $T$. 


\begin{tabular}{|c|c|c|c|c|c|c|c|c|}
\hline & \multicolumn{4}{|c|}{$A_{S}$} & & \multicolumn{3}{|c|}{$B_{S}$} \\
\hline & & $n=0$ & $n=1,3$ & $n=2$ & & $n=0$ & $n=1,3$ & $n=2$ \\
\hline \multirow{3}{*}{ (a) } & & 3 & -1 & -1 & \multirow{3}{*}{$\left(M_{m, l}^{2}+M_{m^{\prime}, l^{\prime}}^{2}\right) \times$} & $5 / 2$ & $-1 / 2$ & $-3 / 2$ \\
\hline & $\frac{1}{3} \times<$ & 2 & 0 & -2 & & 2 & 0 & -1 \\
\hline & & $5 / 2$ & $-1 / 2$ & $-3 / 2$ & & $9 / 4$ & $-1 / 4$ & $-7 / 4$ \\
\hline \multirow{3}{*}{ (b) } & \multirow{3}{*}{\multicolumn{2}{|c|}{0}} & \multirow{3}{*}{0} & \multirow{3}{*}{0} & \multirow{3}{*}{$-\left(M_{m, l}^{2}+M_{m^{\prime}, l^{\prime}}^{2}\right) \times$} & $5 / 2$ & $-1 / 2$ & $-3 / 2$ \\
\hline & & & & & & 2 & 0 & -1 \\
\hline & & & & & & $9 / 4$ & $-1 / 4$ & $-7 / 4$ \\
\hline
\end{tabular}

Table 1: Scalar functions $A_{S}$ and $B_{S}$ for scalar matter loops, as defined via Eq. (5.5), corresponding to the diagrams $(a)$ and $(b)$ of Fig. 1. These are computed in dimensional regularization. We give the results for scalars satisfying the four types of boundary conditions, labeled by $n=0,1,2,3$, allowed on the chiral square compactification of Ref. [10]. For a given $n$ and for each diagram, there are three possible cases depending on KK-parity and whether $m-m^{\prime}$ is even or odd, as listed in Eq. (2.30): the first two cases correspond to even-even mixings with $m-m^{\prime}$ even and $m-m^{\prime}$ odd, and the third case to odd-odd mixings. All KK-parity violating transitions vanish. In all cases the gauge violating coefficient $B_{S}$ vanishes when both diagrams are added.

Clearly, for given $(m, l)$ and $\left(m^{\prime}, l^{\prime}\right)$ only a finite number of states contribute to the KK sum. Also, since $m_{1}$ and $l_{1}$ must be integers, only when $(-1)^{m+l+m^{\prime}+l^{\prime}}=+1$ can the result be nonvanishing, in accordance with the KK-parity assignment $(-1)^{m+l}$ for states with quantum numbers $(m, l)$. Like for the KK-number conserving terms, the (logarithmically) divergent contribution for each nonvanishing term in the sum is proportional to $m_{1}^{2}+l_{1}^{2}$ (in dimensional regularization). To obtain the correct KK-number violating structure, it is necessary to contract with the external propagators, as in Eq. (5.1). In this process, all the crossed terms, such as $m m^{\prime}$ or $l l^{\prime}$ cancel out and only terms proportional to $M_{m, l}^{2}+M_{m^{\prime}, l^{\prime}}^{2}$ survive.

A straightforward computation allows us to write the KK-number violating contribution to the two-point function in momentum space as

$$
\left(\frac{1}{4 L^{2}}\right) G_{\mu, p}^{\alpha(j, k)}\left\{-i \frac{g_{4}^{2}}{16 \pi^{2}} T(\Phi) \delta_{a b} \Gamma\left(\frac{\epsilon}{2}\right)\left[A_{S}\left(p^{2} \eta_{\alpha \beta}-p_{\alpha} p_{\beta}\right)-B_{S} \eta_{\alpha \beta}\right]\right\} G_{\nu, p}^{\beta\left(j^{\prime}, k^{\prime}\right)}
$$

where $\epsilon=4-D$ and $\operatorname{Tr}\left(T^{a} T^{b}\right)=T(\Phi) \delta_{a b}$, with $T(\Phi)=1 / 2$ for matter in the fundamental representation of $S U(N)$. The scalar functions $A_{S}, B_{S}$ for the four types of boundary conditions are given in Table 1, and the explicit factor of $1 /\left(4 L^{2}\right)$ in Eq. (5.5) should be identified with the one appearing in Eq. (2.18), while the remaining factor of $1 / L^{2}$ was absorbed in the $4 \mathrm{D}$ gauge coupling, $g_{4}^{2}=g_{6}^{2} / L^{2}$.

We see that for each type of boundary condition $(n=0,1,2$ or 3 ) obeyed by the scalar running in the loop, there are three different cases, that we ordered as in Eq. (2.30). This is precisely the KK-number violating structure that arises from quadratic operators involving fields satisfying $n=0$ boundary conditions (the gauge field), localized at the points $(0,0),(L, L)$ and 
$(0, L)$, as discussed in Section 2.3. Notice that the above three cases are distinct precisely as a result of the operator localized at $(0, L)$, proportional to $c$ in Eq. (2.21).

For the diagram involving the interactions between two scalars and a single gauge boson, the two-point function in momentum space is

$$
\left\langle A_{\mu}^{(j, k)} A_{\nu}^{\left(j^{\prime}, k^{\prime}\right)}\right\rangle^{(a)}=\left(\frac{1}{4 L^{2}}\right)^{2}\left(\frac{1}{4}\right)^{2} \sum_{m, l} \sum_{m^{\prime}, l^{\prime}} G_{\mu, p}^{\alpha(j, k ; m, l)}\left[\left\langle A_{\alpha}^{(m, l)} A_{\beta}^{\left(m^{\prime}, l^{\prime}\right)}\right\rangle_{\mathrm{amp}}^{(a)}\right] G_{\nu, p}^{\beta\left(m^{\prime}, l^{\prime} ; j^{\prime}, k^{\prime}\right)},
$$

where now we get a factor $(1 / 4)^{2}$ due to the two vertices, and the amputated function of Figure $1(a)$ is

$$
\begin{gathered}
\left\langle A_{\alpha}^{(m, l)} A_{\beta}^{\left(m^{\prime}, l^{\prime}\right)}\right\rangle_{\mathrm{amp}}^{(a)}=-g_{6}^{2} i^{2} \operatorname{Tr}\left(T^{a} T^{b}\right) \sum_{m_{1}, l_{1}} \sum_{m_{1}^{\prime}, l_{1}^{\prime}} \int \frac{d^{D} k}{(2 \pi)^{D}}(2 k+p)_{\alpha}(2 k+p)_{\beta} \\
\times G_{k+p, n}^{\left(m_{1}, l_{1} ; m_{1}^{\prime}, l_{1}^{\prime}\right)} G_{k, n}^{\left(m_{2}^{\prime}, l_{2}^{\prime} ; m_{2}, l_{2}\right)}
\end{gathered}
$$

where it is understood that, due to momentum conservation at each vertex, $m_{2}=m_{1}-m$, $l_{2}=l_{1}-l, m_{2}^{\prime}=m_{1}^{\prime}-m^{\prime}$ and $l_{2}^{\prime}=l_{1}^{\prime}-l^{\prime}$. Noting that $M_{m_{2}^{\prime}, l_{2}^{\prime}}^{2}$ depends on $m_{2}^{\prime}, l_{2}^{\prime}$ only through $m_{2}^{\prime 2}+l_{2}^{\prime 2}$, and is therefore invariant under exchange of $m_{2}^{\prime}$ and $l_{2}^{\prime}$ and/or a change in their signs, we have

$$
G_{k, n}^{\left(m_{2}^{\prime}, l_{2}^{\prime} ; m_{2}, l_{2}\right)}=\frac{i}{k^{2}-M_{m_{2}^{\prime}, l_{2}^{\prime}}^{2}} \hat{\delta}\left(m_{2}^{\prime}, l_{2}^{\prime} ; m^{\prime}, l^{\prime} ; n\right)=\frac{i}{k^{2}-M_{m^{\prime}, l^{\prime}}^{2}} \hat{\delta}\left(m_{2}^{\prime}, l_{2}^{\prime} ; m^{\prime}, l^{\prime} ; n\right)
$$

which shows that the integrand in Eq. (5.7) depends on $m_{1}^{\prime}, l_{1}^{\prime}$ only through the generalized $\delta$-functions defined in Eq. (2.14). We can then do the sum over $m_{1}^{\prime}, l_{1}^{\prime}$ using the identity [see Eq. (A.17) with $n_{1}=n_{2}=n$ ]

$$
\begin{aligned}
\sum_{m_{1}^{\prime}, l_{1}^{\prime}} \hat{\delta} & \left(m_{1}, l_{1} ; m_{1}^{\prime}, l_{1}^{\prime} ; n\right) \hat{\delta}\left(m_{2}^{\prime}, l_{2}^{\prime} ; m_{2}, l_{2} ; n\right) \\
= & \hat{\delta}\left(m, l ; m^{\prime}, l^{\prime} ; 0\right)+e^{i \theta} \hat{\delta}\left(m-m_{1}-l_{1}, l-l_{1}+m_{1} ; m^{\prime}, l^{\prime} ; 0\right) \\
& \quad+e^{2 i \theta} \hat{\delta}\left(m-2 m_{1}, l-2 l_{1} ; m^{\prime}, l^{\prime} ; 0\right)+e^{3 i \theta} \hat{\delta}\left(m-m_{1}+l_{1}, l-l_{1}-m_{1} ; m^{\prime}, l^{\prime} ; 0\right)
\end{aligned}
$$

where $\theta=n \pi / 2$. We see that the first term has the same structure as the tree-level propagator. It is also independent of the loop momenta, $m_{1}, l_{1}$, so that the corresponding KK sum leads to a power-law divergence. The remaining terms give KK violating transitions that, as we will see, correspond to localized operators. In order to compare to diagram (b) as computed before, we should contract with the external propagators and perform the KK sums. The KK number preserving term just gives

$$
\sum_{m, l} \sum_{m^{\prime}, l^{\prime}} \hat{\delta}(j, k ; m, l ; 0) \hat{\delta}\left(m, l ; m^{\prime}, l^{\prime} ; 0\right) \hat{\delta}\left(m^{\prime}, l^{\prime} ; j^{\prime}, k^{\prime} ; 0\right)=16 \hat{\delta}\left(j, k ; j^{\prime}, k^{\prime} ; 0\right) .
$$

The contraction of the external propagators with the last three, KK-number violating terms in Eq. (5.9), do not alter the KK violating structure, and simply give an overall factor of 16 . 
Thus, unlike for diagrams with the topology of diagram $(b)$, for diagrams with the topology of diagram $(a)$ it is sufficient to compute the amputated diagram, while keeping in mind that the contraction with external propagators produces a factor of 16 that cancels against some of the factors in the two-point function. The net effect is that for these diagrams one should include a factor of $1 /\left(4 L^{2}\right)$ where the length scale $L$ is absorbed by the 4 D gauge coupling via $g_{4}^{2}=g_{6}^{2} / L^{2}$. A straightforward computation allows us to express the KK violating contributions to diagram (a) as in Eq. (5.5), with scalar coefficients $A_{S}$ and $B_{S}$ as given in Table 1 .

We see that after adding the two diagrams $(a)$ and $(b)$, the "gauge violating" contribution proportional to $\eta_{\alpha \beta}$ cancels out. Therefore, at one-loop order, the scalar gauge interactions give a contribution to the two point function which is equivalent to the effect of the localized operator

$$
\frac{1}{4} \delta_{c_{S}}(z) \times\left(-\frac{1}{4} \hat{r}_{S} L^{2} F_{\mu \nu}^{a} F^{\mu \nu a}\right)
$$

where $\delta_{c}(z)$ stands for the Dirac delta-functions at the conical singularities, as defined in Eq. (2.21), and the factor of $1 / 4$ accounts for universal KK wavefunction enhancements. Here we wrote the dimensionful coefficient in Eq. (2.21) as $r_{S}=\hat{r}_{S} L^{2}$, so that the scalar contribution is

$$
\hat{r}_{S}=\frac{2}{3} \times \frac{g_{4}^{2}}{16 \pi^{2}} T(\Phi) \Gamma\left(\frac{\epsilon}{2}\right) \times\left\{\begin{array}{rl}
5 / 8 \\
-1 / 8 \\
-3 / 8
\end{array}, \quad c_{S}=\left\{\begin{array}{cl}
2 / 5 & \text { for } \mathrm{n}=0 \\
2 & \text { for } \mathrm{n}=1,3 \\
-2 / 3 & \text { for } \mathrm{n}=2
\end{array} .\right.\right.
$$

This shows explicitly that the logarithmic divergences that appear in the chiral square compactification renormalize operators precisely at the three conical singularities.

\subsubsection{Fermionic Matter}

We now turn to fermionic matter. We restrict ourselves to $6 \mathrm{D}$ fermions containing a zero-mode (i.e. we do not consider $n=2$ boundary conditions).

We use the same labeling conventions as in Figure 1, as we will do throughout this paper. As discussed in detail in the previous section, we may concentrate on the amputated fermion loop diagram, including a factor of $1 / 4$ :

$$
-\left(\frac{1}{4}\right) g_{4}^{2} i^{2} \operatorname{Tr}\left(T^{a} T^{b}\right) \sum_{m_{1}, l_{1}} \sum_{m_{1}^{\prime}, l_{1}^{\prime}} \int \frac{d^{D} k}{(2 \pi)^{D}} \operatorname{Tr}\left\{\Gamma_{\beta} G_{k+p}^{ \pm,\left(m_{1}, l_{1} ; m_{1}^{\prime}, l_{1}^{\prime}\right)} \Gamma_{\alpha} G_{k}^{ \pm,\left(m_{2}^{\prime}, l_{2}^{\prime} ; m_{2}, l_{2}\right)} P_{\mp}\right\}
$$

where the fermion propagator was given in Eq. (3.3). Also, as explained in detail in the scalar loop calculation described before, we separate the result into a KK-number preserving contribution that renormalizes bulk operators and a KK-number violating contribution that renormalize localized operators.

The trace in Eq. (5.12) can be separated into parts involving the non-compact dimensions

$$
(k+p)^{\lambda} k^{\rho} \operatorname{Tr}\left\{\Gamma_{\beta} \Gamma_{\lambda} \Gamma_{\alpha} \Gamma_{\rho} P_{L, R} P_{\mp}\right\}=2\left[(k+p)_{\alpha} k_{\beta}+(k+p)_{\beta} k_{\alpha}-k \cdot(k+p) \eta_{\alpha \beta}\right],
$$


and parts involving the compact dimensions (denoted by indices $i, j$ )

$$
\begin{aligned}
(k+p)_{i} k_{j} \operatorname{Tr}\left\{\Gamma_{\beta} \Gamma^{i} \Gamma_{\alpha} \Gamma^{j} P_{L} P_{\mp}\right\} & =r_{m_{1}, l_{1}} r_{m_{2}^{\prime}, l_{2}^{\prime}}^{*} M_{m_{1}, l_{1}} M_{m_{2}^{\prime}, l_{2}^{\prime}} \operatorname{Tr}\left\{\Gamma_{\beta} \Gamma^{4} \Gamma_{\alpha} \Gamma^{4} P_{L} P_{\mp}\right\} \\
& =2 \eta_{\alpha \beta} r_{m_{1}, l_{1}} r_{m_{2}, l_{2}^{\prime}}^{*} M_{m_{1}, l_{1}} M_{m_{2}^{\prime}, l_{2}^{\prime}}, \\
(k+p)_{i} k_{j} \operatorname{Tr}\left\{\Gamma_{\beta} \Gamma^{i} \Gamma_{\alpha} \Gamma^{j} P_{R} P_{\mp}\right\} & =r_{m_{1}, l_{1}}^{*} r_{m_{2}^{\prime}, l_{2}^{\prime}} M_{m_{1}, l_{1}} M_{m_{2}^{\prime}, l_{2}^{\prime}} \operatorname{Tr}\left\{\Gamma_{\beta} \Gamma^{4} \Gamma_{\alpha} \Gamma^{4} P_{R} P_{\mp}\right\} \\
& =2 \eta_{\alpha \beta} r_{m_{1}, l_{1}}^{*} r_{m_{2}, l_{2}^{\prime}} M_{m_{1}, l_{1}} M_{m_{2}^{\prime}, l_{2}^{\prime}},
\end{aligned}
$$

where we used the useful identities 10$]$

$$
\begin{aligned}
& \Gamma^{5} P_{L} P_{ \pm}= \pm i \Gamma^{4} P_{L} P_{ \pm} \\
& \Gamma^{5} P_{R} P_{ \pm}=\mp i \Gamma^{4} P_{R} P_{ \pm}
\end{aligned}
$$

to replace $\Gamma^{5}$ in favor of $\Gamma^{4}$. We also used $k_{4}+p_{4}=-m_{1} / R, k_{5}+p_{5}=-l_{1} / R, k_{4}=-m_{2}^{\prime} / R$ and $k_{5}=-l_{2}^{\prime} / R$. The momentum dependent phases, $r_{j, k}$, were defined in Eq. (2.27). Notice that the $6 \mathrm{D}$ chirality operator $\bar{\Gamma}$ contained in $P_{ \pm}$gives a nonvanishing contribution to Eqs. (5.14) and (5.15).

We express the result associated with the KK-number violating terms as

$$
\left(\frac{1}{4 L^{2}}\right) G_{\mu, p}^{\alpha(j, k)}\left\{-i \frac{g_{4}^{2}}{16 \pi^{2}} T(\Psi) \delta_{a b} \Gamma\left(\frac{\epsilon}{2}\right)\left[A_{F}\left(p^{2} \eta_{\alpha \beta}-p_{\alpha} p_{\beta}\right)-B_{F} \eta_{\alpha \beta}\right]\right\} G_{\nu, p}^{\beta\left(j^{\prime}, k^{\prime}\right)},
$$

and find that for all KK-parity preserving transitions $A_{F}=4 / 3$ and $B_{F}=0$. As for the scalar loops, the "gauge violating" term proportional to $\eta_{\alpha \beta}$ vanishes. The equivalent localized operator is in this case

$$
\frac{1}{4} \delta_{c_{F}}(z) \times\left(-\frac{1}{4} \hat{r}_{F} L^{2} F_{\mu \nu}^{a} F^{\mu \nu a}\right)
$$

where $\delta_{c}(z)$ was defined in Eq. (2.21), the factor of $1 / 4$ accounts for universal KK wavefunction enhancements, and

$$
\hat{r}_{F}=\frac{2}{3} \times \frac{g_{4}^{2}}{16 \pi^{2}} T(\Psi) \Gamma\left(\frac{\epsilon}{2}\right), \quad c_{F}=0 .
$$

Note that the fermion loop does not induce an operator localized at $(0, L)$, i.e. the three cases defined in (2.30) give the same result.

\subsubsection{Gauge Self-Interactions}

We consider now the one-loop corrections arising from the self-interactions in a non-abelian gauge theory. The diagrams are shown in Figure 3. In terms of the momentum space propagators for the gauge bosons, the scalar adjoints and the ghost fields, given in Eqs. (4.4), (4.6) and (4.10), they are:

$$
(c)=\left(\frac{1}{4}\right) \frac{g_{4}^{2}}{2} f^{a d l} f^{b d l} \sum_{m_{1}, l_{1}} \sum_{m_{1}^{\prime}, l_{1}^{\prime}} \int \frac{d^{D} k}{(2 \pi)^{D}}\left[\eta_{\alpha \delta}(p-k)_{\lambda}+\eta_{\delta \lambda}(2 k+p)_{\alpha}-\eta_{\lambda \alpha}(k+2 p)_{\delta}\right]
$$




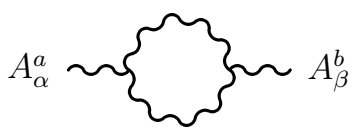

(c)

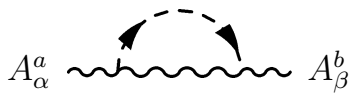

(f)

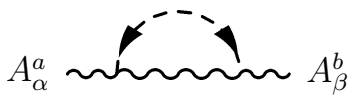

(i)

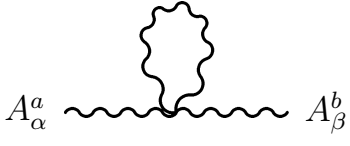

(d)

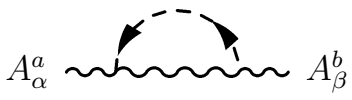

(g)

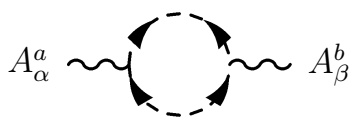

(j)

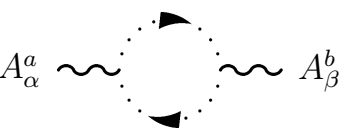

(e)

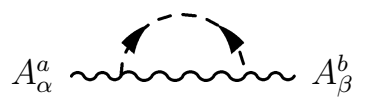

(h)

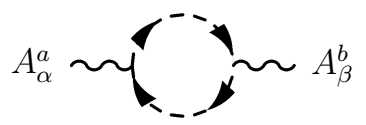

(k)

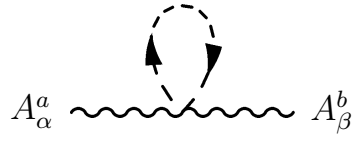

(1)

Figure 3: Gauge self-energy diagrams at one-loop in the 6D theory: $(c),(d)$ gauge loops; $(e)$ ghost loop; and $(f)-(l)$ loops associated with the $A_{4}$ and $A_{5}$ components of the $6 \mathrm{D}$ gauge field. For the latter, the arrows represent the propagation of $A_{+}=A_{4}+i A_{5}$.

$$
\begin{aligned}
& \times G_{\left(m_{1}, l_{1} ; m_{1}^{\prime}, l_{1}^{\prime}\right)}^{\lambda \lambda^{\prime}, k+p}\left[\eta_{\lambda^{\prime} \beta}(k+2 p)_{\delta^{\prime}}-\eta_{\delta^{\prime} \lambda^{\prime}}(2 k+p)_{\beta}+\eta_{\beta \delta^{\prime}}(k-p)_{\lambda^{\prime}}\right] G_{\left(m_{2}^{\prime}, l_{2}^{\prime} ; m_{2}, l_{2}\right)}^{\delta \delta^{\prime}, k}, \\
& (d)=-i \frac{g_{4}^{2}}{2} f^{a d l} f^{b d l} \sum_{m_{1}, l_{1}} \int \frac{d^{D} k}{(2 \pi)^{D}}\left[2 \eta_{\alpha \beta} \eta_{\delta \delta^{\prime}}-\eta_{\alpha \delta} \eta_{\beta \delta^{\prime}}-\eta_{\alpha \delta^{\prime}} \eta_{\beta \delta}\right] G_{\left(m_{1}, l_{1} ; m_{1}^{\prime}, l_{1}^{\prime}\right)}^{\delta \delta^{\prime}, k} \\
& (e)=-\left(\frac{1}{4}\right) g_{4}^{2} f^{a l d} f^{b d l} \sum_{m_{1}, l_{1}} \sum_{m_{1}^{\prime}, l_{1}^{\prime}} \int \frac{d^{D} k}{(2 \pi)^{D}}(k+p)_{\alpha} G_{\xi, k+p}^{\left(m_{1}, l_{1} ; m_{1}^{\prime}, l_{1}^{\prime}\right)} k_{\beta} G_{\xi, k}^{\left(m_{2}^{\prime}, l_{2}^{\prime} ; m_{2}, l_{2}\right)} \\
& (f)+(g)=\left(\frac{1}{4}\right)\left(\frac{1}{2}\right)^{2} g_{4}^{2} f^{a l d} f^{b d l} \sum_{m_{1}, l_{1}} \sum_{m_{1}^{\prime}, l_{1}^{\prime}} \int \frac{d^{D} k}{(2 \pi)^{D}}\left[r_{m^{\prime}, l^{\prime}} M_{m^{\prime}, l^{\prime}}-r_{m_{2}^{\prime}, l_{2}^{\prime}} M_{m_{2}^{\prime}, l_{2}^{\prime}}\right] \\
& \times G_{++, k+p}^{\left(m_{1}, l_{1} ; m_{1}^{\prime}, l_{1}^{\prime}\right)}\left[r_{m, l}^{*} M_{m, l}-r_{m_{2}, l_{2}}^{*} M_{m_{2}, l_{2}}\right] G_{\alpha \beta, k}^{\left(m_{2}^{\prime}, l_{2}^{\prime} ; m_{2}, l_{2}\right)}+\text { h.c. , } \\
& (h)+(i)=\left(\frac{1}{4}\right)\left(\frac{1}{2}\right)^{2} g_{4}^{2} f^{a l d} f^{b d l} \sum_{m_{1}, l_{1}} \sum_{m_{1}^{\prime}, l_{1}^{\prime}} \int \frac{d^{D} k}{(2 \pi)^{D}}\left[r_{m^{\prime}, l^{\prime}} M_{m^{\prime}, l^{\prime}}-r_{m_{2}^{\prime}, l_{2}^{\prime}} M_{m_{2}^{\prime}, l_{2}^{\prime}}\right] \\
& \times G_{+-, k+p}^{\left(m_{1}, l_{1} ; m_{1}^{\prime}, l_{1}^{\prime}\right)}\left[r_{m, l} M_{m, l}-r_{m_{2}, l_{2}} M_{m_{2}, l_{2}}\right] G_{\alpha \beta, k}^{\left(m_{2}^{\prime}, l_{2}^{\prime} ; m_{2}, l_{2}\right)}+\text { h.c. , }
\end{aligned}
$$




$$
\begin{aligned}
(j)=\left(\frac{1}{4}\right) \frac{g_{4}^{2}}{4} f^{a l d} f^{b d l} \sum_{m_{1}, l_{1}} \sum_{m_{1}^{\prime}, l_{1}^{\prime}} \int \frac{d^{D} k}{(2 \pi)^{D}}(2 k+p)_{\alpha} & (2 k+p)_{\beta} \\
& \times G_{+-, k+p}^{\left(m_{1}, l_{1} ; m_{1}^{\prime}, l_{1}^{\prime}\right)} G_{-+, k}^{\left(m_{2}^{\prime}, l_{2}^{\prime} ; m_{2}, l_{2}\right)}, \\
(k)=\left(\frac{1}{4}\right) \frac{g_{4}^{2}}{4} f^{a l d} f^{b d l} \sum_{m_{1}, l_{1}} \sum_{m_{1}^{\prime}, l_{1}^{\prime}} \int \frac{d^{D} k}{(2 \pi)^{D}}(2 k+p)_{\alpha}(2 k+p)_{\beta} & \times G_{++, k+p}^{\left(m_{1}, l_{1} ; m_{1}^{\prime}, l_{1}^{\prime}\right)} G_{++, k}^{\left(m_{2}, l_{2} ; m_{2}^{\prime}, l_{2}^{\prime}\right)}, \\
(l)=i g_{4}^{2} f^{a d l} f^{b d l} \eta_{\alpha \beta} \sum_{m_{1}, l_{1}} \int \frac{d^{D} k}{(2 \pi)^{D}} G_{++, k}^{\left(m_{1}, l_{1} ; m_{1}^{\prime}, l_{1}^{\prime}\right)}, &
\end{aligned}
$$

where we included a factor $1 / 4$ for the diagrams involving trilinear interactions, as explained in subsection 5.1.1. It is similarly understood that the diagrams involving a quartic interaction should be contracted with the external propagators in order to obtain the correct KK-number structure in the two-point function [see discussion after Eq. (5.4)]. The color factors are

$$
f^{a d l} f^{b d l}=C_{2}(A) \delta_{a b}
$$

where $C_{2}(A)=N$ for a $S U(N)$ group. A lengthy but straightforward calculation allows us to write the leading logarithmic divergences of diagrams $(c)-(l)$ as

$$
-i \frac{g_{4}^{2}}{16 \pi^{2}} C_{2}(A) \delta_{a b} \Gamma\left(\frac{\epsilon}{2}\right)\left[A_{G}\left(p^{2} \eta^{\mu \nu}-p^{\mu} p^{\nu}\right)-B_{G} \eta^{\mu \nu}\right]
$$

where the scalar functions $A_{G}$ and $B_{G}$ are presented in Table 2.

Note that the $p^{2}$ terms in $B_{G}$ cancel between the ghost loop diagram and the diagram involving trilinear gauge self-interactions, as happens in 4D QCD. Therefore, the "gauge violating" terms in Eq. (5.30), proportional to $\eta_{\alpha \beta}$, are proportional to the KK masses, thus preserving the unbroken $4 \mathrm{D}$ gauge invariance. Diagrams $(f)-(i)$ involving the coupling of a single scalar to two gauge fields are directly related to the higher dimensional Higgs mechanism. Also the divergent parts of diagrams $(h)-(j)$ vanish. This results from a cancellation between the two real scalar degrees of freedom in $A_{+}$, as seen in the form of the propagator $G_{+-, p}^{\left(m_{1}, l_{1} ; m_{1}^{\prime}, l_{1}^{\prime}\right)}$ of Eq. (4.6). Even though for arbitrary $\xi$ the two real scalar degrees of freedom have different masses, the $\xi$-dependence cancels out in the infinite terms. Note also that diagrams $(k)$ and $(l)$ with $\xi=1$, when the two real degrees of freedom in $A_{+}$have the same mass, agree with the results derived for a complex scalar minimally coupled to a gauge field and satisfying $n=3$ boundary conditions, as given in Table 1 .

Adding the results of Table 2 we find that the contribution to the $6 \mathrm{D}$ gauge self-interactions is given by

$$
A_{G}=\frac{1}{2} \xi \times\left\{\begin{array}{l}
3 \\
2 \\
5 / 2
\end{array}-\left\{\begin{array}{l}
41 / 6 \\
13 / 3 \\
67 / 12
\end{array} \quad, \quad B_{G}=\frac{1}{4}(3+\xi)\left(M_{m, l}^{2}+M_{m^{\prime}, l^{\prime}}^{2}\right) \times\left\{\begin{array}{l}
3 \\
2 \\
5 / 2
\end{array}\right.\right.\right.
$$




\begin{tabular}{|c|c|c|c|c|c|c|c|}
\hline & \multicolumn{2}{|c|}{$A_{G}$} & \multicolumn{5}{|c|}{$B_{G}$} \\
\hline (c) & $\left(\frac{1}{2} \xi-\frac{7}{3}\right) \times$ & $\begin{array}{l}3 \\
2 \\
5 / 2\end{array}$ & $-\frac{1}{4} p^{2} \times\{$ & $\begin{array}{l}3 \\
2 \\
5 / 2\end{array}+\frac{3}{4}\left(4+\xi+\xi^{2}\right)\left(M_{m, l}^{2}\right.$ & $-M_{m^{\prime}, l}^{2}$ & 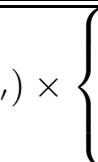 & $\begin{array}{l}5 / 4 \\
1 \\
9 / 8\end{array}$ \\
\hline (d) & \multicolumn{2}{|l|}{0} & \multicolumn{5}{|c|}{$-\frac{3}{4}\left(3+\xi^{2}\right)\left(M_{m, l}^{2}+M_{m^{\prime}, l^{\prime}}^{2}\right) \times\left\{\begin{array}{l}5 / 4 \\
1 \\
9 / 8\end{array}\right.$} \\
\hline (e) & \multicolumn{2}{|c|}{$\frac{1}{6} \times\left\{\begin{array}{l}3 \\
2 \\
5 / 2\end{array}\right.$} & \multicolumn{3}{|c|}{$\frac{1}{4} p^{2} \times\left\{\begin{array}{l}3 \\
2 \\
5 / 2\end{array}-\frac{1}{2} \xi\left(M_{m, l}^{2}+M_{m^{\prime},}^{2}\right.\right.$} & \multicolumn{2}{|c|}{$\begin{array}{l}5 / 4 \\
1 \\
9 / 8\end{array}$} \\
\hline$(\mathrm{f})+(\mathrm{g})$ & \multicolumn{2}{|l|}{0} & \multicolumn{2}{|r|}{$-\frac{1}{8}(3+\xi)\left(M_{m, l}^{2}+M_{m^{\prime}, l^{\prime}}^{2}\right) \times\{$} & $\begin{array}{l}-7 / 2 \\
-2 \\
-11\end{array}$ & & \\
\hline$(\mathrm{h})+(\mathrm{i})$ & \multicolumn{2}{|l|}{0} & \multicolumn{5}{|c|}{0} \\
\hline (j) & \multicolumn{2}{|l|}{0} & \multicolumn{5}{|c|}{0} \\
\hline (k) & \multicolumn{2}{|c|}{$-\frac{1}{6} \times\left\{\begin{array}{l}2 \\
0 \\
1\end{array}\right.$} & \multicolumn{2}{|r|}{$\frac{1}{4}(1+\xi)\left(M_{m, l}^{2}+M_{m^{\prime}, l^{\prime}}^{2}\right) \times\{$} & \multicolumn{3}{|c|}{$\begin{array}{c}-1 \\
0 \\
-1 / 2\end{array}$} \\
\hline (1) & \multicolumn{2}{|l|}{0} & \multicolumn{2}{|r|}{$-\frac{1}{4}(1+\xi)\left(M_{m, l}^{2}+M_{m^{\prime}, l^{\prime}}^{2}\right) \times\{$} & $\left\{\begin{array}{c}-1 \\
0 \\
-1 /\end{array}\right.$ & & \\
\hline
\end{tabular}

Table 2: Scalar functions $A_{G}$ and $B_{G}$ in the non-abelian gauge sector, as defined via Eq. (5.30), corresponding to the diagrams $(c)-(l)$ of Fig. 3. For each diagram, the first two cases correspond to even-even mixings with $m-m^{\prime}$ even and $m-m^{\prime}$ odd, and the third to odd-odd mixings, as listed in Eq. (2.30).

which, together with Eq. (5.30), determines the KK-number violating contribution to the gauge boson two-point function arising from the gauge self-interactions in non-abelian gauge theories.

\subsubsection{Mass Shifts and Localized Operators}

It should be noted that the parameters $A_{G}$ and $B_{G}$ given in Eq. (5.31), are gauge dependent. In addition, as already mentioned, we find a "gauge violating" term proportional to $\eta_{\mu \nu}$, i.e. $B_{G} \neq 0$. This term would arise from the localized operators [see Eq. (1.4)]

$$
\mathcal{O}_{1,2}=A^{\mu} \partial_{+} \partial_{-} A_{\mu}+\text { h.c. }
$$

Although these operators do not break the 4D gauge invariance associated with the zero-mode gauge field, they are certainly not invariant under the set of $6 \mathrm{D}$ gauge transformations left unbroken by the chiral square compactification [i.e. those generated by a gauge transformation parameter satisfying $n=0$ boundary conditions, as does $A_{\mu}$.] Nevertheless, given the fact that 
the kinetic term renormalization parameter, $A_{G}$, is $\xi$-dependent, such terms are necessary to ensure that physical quantities such as the mass shifts of the massive KK modes are gauge invariant.

Indeed, the KK diagonal components in Eq. (5.30) give the leading contribution to the KK mass shifts. For KK-parity even states, $(-1)^{m+l}=+1$, case 1 in Eq. (5.31) gives a self-energy

$$
-i \frac{g_{4}^{2}}{16 \pi^{2}} C_{2}(A) \delta_{a b} \Gamma\left(\frac{\epsilon}{2}\right)\left[\left(\frac{3}{2} \xi-\frac{41}{6}\right)\left(p^{2} \eta_{\mu \nu}-p_{\mu} p_{\nu}\right)-\frac{3}{2}(3+\xi) M_{m, l}^{2} \eta_{\mu \nu}\right],
$$

which leads to a mass shift

$$
\begin{aligned}
\delta M_{m, l}^{2} & =\frac{g_{4}^{2}}{16 \pi^{2}} C_{2}(A) \Gamma\left(\frac{\epsilon}{2}\right)\left[\frac{3}{2}(3+\xi)-\left(\frac{3}{2} \xi-\frac{41}{6}\right)\right] M_{m, l}^{2} \\
& =\frac{34}{3} \times \frac{g_{4}^{2}}{16 \pi^{2}} C_{2}(A) \Gamma\left(\frac{\epsilon}{2}\right) M_{m, l}^{2} .
\end{aligned}
$$

Similarly, for KK-parity odd states, $(-1)^{m+l}=-1$, case 3 in Eq. (5.31) gives a (diagonal) self-energy

$$
-i \frac{g_{4}^{2}}{16 \pi^{2}} C_{2}(A) \delta_{a b} \Gamma\left(\frac{\epsilon}{2}\right)\left[\left(\frac{5}{4} \xi-\frac{67}{12}\right)\left(p^{2} \eta_{\mu \nu}-p_{\mu} p_{\nu}\right)-\frac{5}{4}(3+\xi) M_{m, l}^{2} \eta_{\mu \nu}\right]
$$

which leads to a mass shift

$$
\delta M_{m, l}^{2}=\frac{28}{3} \times \frac{g_{4}^{2}}{16 \pi^{2}} C_{2}(A) \Gamma\left(\frac{\epsilon}{2}\right) M_{m, l}^{2} .
$$

We see that the $\xi$ dependence disappears from the physical mass shifts, as expected.

The presence of the gauge non-invariant operator associated with $B_{G}$ and the $\xi$-dependence of $A_{G}$ and $B_{G}$ go hand in hand. By an appropriate field redefinition, one should be able to absorb the $\eta_{\mu \nu}$ term into purely gauge invariant operators, with gauge independent coefficients. ${ }^{6}$ Notice that the $6 \mathrm{D}$ gauge violating term vanishes for $\xi=-3$. Therefore, in this particular gauge no further field redefinition is necessary. The induced localized operators are then

$$
\frac{1}{4} \delta_{c_{G}}(z) \times\left(-\frac{1}{4} \hat{r}_{G} L^{2} F_{\mu \nu}^{a} F^{\mu \nu a}\right),
$$

where $\delta_{c}(z)$ was defined in Eq. (2.21), the factor of $1 / 4$ accounts for universal KK wavefunction enhancements, and

$$
\hat{r}_{G}=-\frac{14}{3} \times \frac{g_{4}^{2}}{16 \pi^{2}} C_{2}(A) \Gamma\left(\frac{\epsilon}{2}\right), \quad c_{G}=\frac{3}{7} .
$$

This should be added to the contributions from scalar and fermion loops given in Eqs. (5.10) and (5.18).

Explicit computation of the KK-number violating one-loop corrections to the trilinear and quartic gauge vertices in the gauge $\xi=-3$ should give precisely the coefficients necessary to provide the non-abelian completion of the kinetic operator Eq. (5.37), but we leave such a check for future work. 


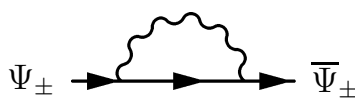

(a)

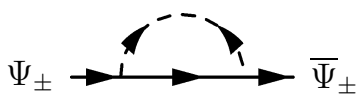

(d)

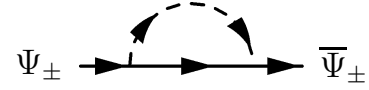

(b)

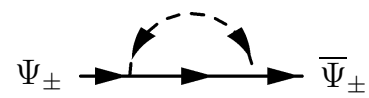

(e)

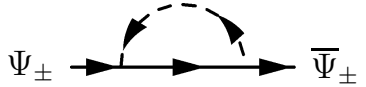

(c)

Figure 4: One-loop fermion self-energy diagrams. These diagrams arise from the interactions with the $6 \mathrm{D}$ gauge sector. The dashed lines with two arrows represent the propagation of $A_{+}$.

\subsection{Fermion Two-Point Function}

We turn now to the one-loop corrections to the fermion two-point function. We discuss first the loops arising from the $6 \mathrm{D}$ gauge interactions. These include interactions with the $4 \mathrm{D}$ gauge fields and interactions with the spinless adjoints. In subsection 5.2.3 we study the corrections arising from Yukawa interactions.

\subsubsection{One-loop Diagrams: Spinor Manipulations in 6D}

The diagrams arising from the 6D gauge interactions are shown in Figure 4 . In terms of the spin- $1 / 2$, spin- 1 and spin-0 propagators given in Eqs. (3.3), (4.4), (4.6), and according to the vertices given in Figure 11, they are given by

$$
\begin{aligned}
& (a)=-\left(\frac{1}{4}\right) g_{4}^{2} C_{2}(\Psi) \sum_{m_{1}, l_{1}} \sum_{m_{1}^{\prime}, l_{1}^{\prime}} \Gamma^{\mu} \int \frac{d^{D} k}{(2 \pi)^{D}} G_{k}^{ \pm\left(m_{2}, l_{2} ; m_{2}^{\prime}, l_{2}^{\prime}\right)} G_{\mu \nu, p-k}^{\left(m_{1}, l_{1} ; m_{1}^{\prime}, l_{1}^{\prime}\right)} \Gamma^{\nu} P_{ \pm} \\
& (b)=-\left(\frac{1}{4}\right) g_{4}^{2} C_{2}(\Psi) \sum_{m_{1}, l_{1}} \sum_{m_{1}^{\prime}, l_{1}^{\prime}} \Gamma^{-} \int \frac{d^{D} k}{(2 \pi)^{D}} G_{k}^{ \pm\left(m_{2}, l_{2} ; m_{2}^{\prime}, l_{2}^{\prime}\right)} G_{++, p-k}^{\left(m_{1}, l_{1} ; m_{1}^{\prime}, l_{1}^{\prime}\right)} \Gamma^{+} P_{ \pm} \\
& (c)=-\left(\frac{1}{4}\right) g_{4}^{2} C_{2}(\Psi) \sum_{m_{1}, l_{1}} \sum_{m_{1}^{\prime}, l_{1}^{\prime}} \Gamma^{+} \int \frac{d^{D} k}{(2 \pi)^{D}} G_{k}^{ \pm\left(m_{2}, l_{2} ; m_{2}^{\prime}, l_{2}^{\prime}\right)} G_{--, p-k}^{\left(m_{1}, l_{1} ; m_{1}^{\prime}, l_{1}^{\prime}\right)} \Gamma^{-} P_{ \pm}, \\
& (d)=-\left(\frac{1}{4}\right) g_{4}^{2} C_{2}(\Psi) \sum_{m_{1}, l_{1}} \sum_{m_{1}^{\prime}, l_{1}^{\prime}} \Gamma^{+} \int \frac{d^{D} k}{(2 \pi)^{D}} G_{k}^{ \pm\left(m_{2}, l_{2} ; m_{2}^{\prime}, l_{2}^{\prime}\right)} G_{-+, p-k}^{\left(m_{1}, l_{1} ; m_{1}^{\prime}, l_{1}^{\prime}\right)} \Gamma^{+} P_{ \pm} \\
& (e)=-\left(\frac{1}{4}\right) g_{4}^{2} C_{2}(\Psi) \sum_{m_{1}, l_{1}} \sum_{m_{1}^{\prime}, l_{1}^{\prime}} \Gamma^{-} \int \frac{d^{D} k}{(2 \pi)^{D}} G_{k}^{ \pm\left(m_{2}, l_{2} ; m_{2}^{\prime}, l_{2}^{\prime}\right)} G_{+-, p-k}^{\left(m_{1}, l_{1} ; m_{1}^{\prime}, l_{1}^{\prime}\right)} \Gamma^{-} P_{ \pm},
\end{aligned}
$$

where a factor of $1 / 4$ was included in each diagram as explained in subsection 5.1.1, and

$$
\Gamma^{ \pm}=\frac{1}{2}\left(\Gamma^{4} \pm i \Gamma^{5}\right) .
$$

\footnotetext{
${ }^{6}$ We thank H. C. Cheng for discussions on this point.
} 
We also chose the directions of the fermion loop momenta $k,\left(m_{2}, l_{2}\right)$ and $\left(m_{2}^{\prime}, l_{2}^{\prime}\right)$ opposite to the convention of Figure 1, so that they are in the direction of the fermion number flow.

We concentrate on some useful remarks relevant in the evaluation of the previous expressions. In particular, we show how to manipulate the $6 \mathrm{D} \Gamma$-matrices efficiently to understand the spinor structure, as well as the KK-number violating structure, of the results.

Starting with diagram $(a)$ and keeping in mind that $\left[P_{ \pm}, P_{L, R}\right]=0$, one obtains a term involving only the momentum along the non-compact dimensions, proportional to

$$
\Gamma^{\mu} k_{\lambda} \Gamma^{\lambda} \Gamma^{\nu} \sum_{m_{1}, l_{1}} \sum_{m_{1}^{\prime}, l_{1}^{\prime}}\left[P_{L} \hat{\delta}\left(m_{2}, l_{2} ; m_{2}^{\prime}, l_{2}^{\prime} ; n_{L}^{ \pm}\right)+P_{R} \hat{\delta}\left(m_{2}, l_{2} ; m_{2}^{\prime}, l_{2}^{\prime} ; n_{R}^{ \pm}\right)\right] \hat{\delta}\left(m_{1}, l_{1} ; m_{1}^{\prime}, l_{1}^{\prime} ; 0\right) P_{ \pm}
$$

and also a term involving the extra-dimensional momenta that has the form of (5.45) with

$$
\begin{aligned}
& k_{\lambda} \Gamma^{\lambda} P_{L} P_{ \pm} \rightarrow-r_{m_{2}, \pm l_{2}} M_{m_{2}, l_{2}} \Gamma^{4} P_{L} P_{ \pm} \\
& k_{\lambda} \Gamma^{\lambda} P_{R} P_{ \pm} \rightarrow-r_{m_{2}, \mp l_{2}} M_{m_{2}, l_{2}} \Gamma^{4} P_{R} P_{ \pm} .
\end{aligned}
$$

To obtain (5.46), we used $k_{4}=-m_{2} / R, k_{5}=-l_{2} / R$, and the identities given in Eq. (5.16) to express $\Gamma^{5}$ in terms of $\Gamma^{4}$. It is understood that the factor in Eq. (5.46) appears under the KK sums. The KK-number violating terms in the KK sums of (5.45) are nonvanishing only when $n_{L}^{ \pm}=0, n_{R}^{ \pm}=0, n_{L}^{ \pm}=2$ or $n_{R}^{ \pm}=2$ [see comments after Eq. (A.17)]. The sums associated with (5.46), involving factors of $m_{2} \pm i l_{2}$, are also non-vanishing in these cases. The evaluation of the momentum integrals and remaining $\Gamma$-matrix algebra proceeds exactly as in $4 \mathrm{D}$.

Turning to the evaluation of diagrams $(b)-(e)$ with the scalars $A_{ \pm}$in the loop, which are characteristic of the $6 \mathrm{D}$ theory, we first notice that the definition (5.44) immediately implies

$$
\left(\Gamma^{+}\right)^{2}=\left(\Gamma^{-}\right)^{2}=0 \text {. }
$$

Also, from the identities given in Eq. (5.16) one can easily see that

$$
\Gamma^{ \pm} P_{L} P_{ \pm}=\Gamma^{\mp} P_{R} P_{ \pm}=0
$$

and also

$$
\begin{gathered}
\Gamma^{ \pm} \Gamma^{\mp} P_{L} P_{ \pm}=-P_{L} P_{ \pm}, \\
\Gamma^{\mp} \Gamma^{ \pm} P_{R} P_{ \pm}=-P_{R} P_{ \pm} .
\end{gathered}
$$

Consider first chirality + fermions. Using the fermion propagator given in Eq. (3.3) and $G_{++}$ given in Eq. (4.6), as well as Eqs. (5.47)-(5.49), one can see that diagram (b) is proportional to

$$
p_{\mu} \Gamma^{\mu} P_{R} P_{+} \sum_{m_{1}, l_{1}} \sum_{m_{1}^{\prime}, l_{1}^{\prime}} \hat{\delta}\left(m_{2}^{\prime}, l_{2}^{\prime} ; m_{2}, l_{2} ;-n_{L}^{+}\right) \hat{\delta}\left(m_{1}, l_{1} ; m_{1}^{\prime}, l_{1}^{\prime} ; 3\right)
$$

so that it only renormalizes the $4 \mathrm{D}$ kinetic term. According to the identity given in Eq. (A.17), the sum in Eq. (5.50) may be written as a sum of generalized $\hat{\delta}$-functions with boundary conditions given by $n_{L}^{+}+3$. As mentioned after Eq. (A.17), the KK-number violating terms vanish unless $n_{L}^{+}=1$, i.e. when $n_{R}^{+}=0$ [see Eq. (3.2)]. 


\begin{tabular}{|c||c|c|}
\hline & $A_{\Psi}$ & $B_{\Psi}$ \\
\hline \hline \multirow{1}{*}{$(\mathrm{a})$} & {$[1+(\xi-1)] \times\left\{\begin{array}{l}3 \\
2 \\
5 / 2\end{array}\right.$} & {$[4+(\xi-1)] \times\left\{\begin{array}{l}3 / 2 \\
1 \\
5 / 4\end{array}\right.$} \\
\hline (c) & $1 \times\left\{\begin{array}{l}-1 \\
0 \\
-1 / 2\end{array}\right.$ & 0 \\
\hline
\end{tabular}

Table 3: Scalar functions $A_{\Psi}$ and $B_{\Psi}$ for the fermion self-energies, as defined via Eq. (5.52), corresponding to the diagrams $(a)$ and $(c)$ of Fig. 6 , when the zero-mode is left-handed. Diagrams $(b),(d)$ and $(e)$ are finite in this case. When the zero-mode is right-handed the results for diagrams $(b)$ and $(c)$ are exchanged. For each diagram, the first two cases correspond to even-even mixings with $m-m^{\prime}$ even and $m-m^{\prime}$ odd, and the third to odd-odd mixings, as listed in Eq. (2.30).

Similarly, diagram $(c)$ is proportional to

$$
p_{\mu} \Gamma^{\mu} P_{L} P_{+} \sum_{m_{1}, l_{1}} \sum_{m_{1}^{\prime}, l_{1}^{\prime}} \hat{\delta}\left(m_{2}^{\prime}, l_{2}^{\prime} ; m_{2}, l_{2} ;-n_{R}^{+}\right) \hat{\delta}\left(m_{1}, l_{1} ; m_{1}^{\prime}, l_{1}^{\prime} ; 1\right),
$$

and the associated KK-number violating terms are non-vanishing only when $n_{R}^{+}=1$, i.e. when $n_{L}^{+}=0$.

For diagrams $(d)$ and $(e)$, it is easy to see that the divergent parts are $\xi$-independent, since they can at most diverge logarithmically. But $G_{+-}$vanishes for $\xi=1$ [see Eq. (4.6)], and therefore these diagrams are finite.

Diagrams $(b)-(e)$ with chirality - fermions on the external lines can be treated similarly.

We summarize our results for the fermion self-energies by considering two cases of phenomenological interest: whether the zero-mode is left- or right-handed. If the zero-mode is left-handed, we find that the KK-number violating contribution to the corresponding fermion two-point function can be written as

$$
i \frac{g_{4}^{2}}{16 \pi^{2}} C_{2}(\Psi) \Gamma\left(\frac{\epsilon}{2}\right)\left\{A_{\Psi} p_{\mu} \Gamma^{\mu} P_{L}-B_{\Psi} \Gamma^{4}\left(r_{m^{\prime}, \mp l^{\prime}} M_{m^{\prime}, l^{\prime}} P_{R}+r_{m, \pm l} M_{m, l} P_{L}\right)\right\} P_{ \pm},
$$

where $C_{2}(\Psi)$ is the eigenvalue of the quadratic Casimir operator in the representation of the fermion $\Psi$, and the scalar function $A_{\Psi}$ and $B_{\Psi}$ are given in Table 3.

When the zero-mode is right-handed, we obtain Eq. (5.52) with $P_{L} \leftrightarrow P_{R}$.

Since the cases $n_{L}=2$ or $n_{R}=2$ do not lead to zero-modes, and are therefore of less phenomenological interest we do not record the results here. However, they can be easily read from Table 3 and the last identity of Appendix $\mathrm{A}$.

\subsubsection{Mass Shifts and Localized Operators}

We can read now the self-energies proper [the diagonal entries in Eq. (5.52)]. For KK-parity 
even modes, we get

$$
i \frac{g_{4}^{2}}{16 \pi^{2}} C_{2}(\Psi) \Gamma\left(\frac{\epsilon}{2}\right)\left\{(3 \xi-1) p_{\mu} \Gamma^{\mu} P_{L}+\frac{3}{2}(3+\xi)\left(p_{4} \Gamma^{4}+p_{5} \Gamma^{5}\right)\right\} P_{ \pm},
$$

where $p_{4}=-m / R$ and $p_{5}=-l / R$. Since only the left-handed fields receive a wavefunction renormalization, after canonical normalization the mass shift is, to lowest order,

$$
\begin{aligned}
\delta M_{m, l} & =\frac{g_{4}^{2}}{16 \pi^{2}} C_{2}(\Psi) \Gamma\left(\frac{\epsilon}{2}\right)\left[\frac{3}{2}(3+\xi)-\frac{1}{2}(3 \xi-1)\right] M_{m, l} \\
& =5 \times \frac{g_{4}^{2}}{16 \pi^{2}} C_{2}(\Psi) \Gamma\left(\frac{\epsilon}{2}\right) M_{m, l}, \quad \text { for }(-1)^{m+l}=+1,
\end{aligned}
$$

and we see that the $\xi$-dependence cancels out, as expected. Notice also that the phases $r_{m, l}$ in (5.52) also appear in the KK mass terms arising from the bulk kinetic term. They can be rotated away by a chiral transformation, or equivalently, absorbed in the definition of the wavefunction profile associated with the right-handed fermions, as done in [10].

For KK-parity odd modes we get the self-energy

$$
i \frac{g_{4}^{2}}{16 \pi^{2}} C_{2}(\Psi) \Gamma\left(\frac{\epsilon}{2}\right)\left\{\left(\frac{5}{2} \xi-\frac{1}{2}\right) p_{\mu} \Gamma^{\mu} P_{L}+\frac{5}{4}(3+\xi)\left(p_{4} \Gamma^{4}+p_{5} \Gamma^{5}\right)\right\} P_{ \pm},
$$

and the corresponding mass shift is

$$
\delta M_{m, l}=4 \times \frac{g_{4}^{2}}{16 \pi^{2}} C_{2}(\Psi) \Gamma\left(\frac{\epsilon}{2}\right) M_{m, l}, \quad \text { for }(-1)^{m+l}=-1 .
$$

It is also interesting to note that Eq. (5.52) corresponds to the localized operator

$$
\frac{1}{4} \delta_{c_{\Psi}}(z) \times\left(\hat{r}_{\Psi} L^{2} i \bar{\Psi}_{+L} \Gamma^{\mu} \partial_{\mu} \Psi_{+L}\right)+\frac{1}{4} \delta_{c_{\Psi}^{\prime}}(z) \times \hat{r}_{\Psi}^{\prime} L^{2}\left[i \bar{\Psi}_{+L} \Gamma^{4} \partial_{-} \Psi_{+R}+\text { h.c. }\right],
$$

where $\delta_{c}(z)$ stands for the Dirac delta-functions at the conical singularities, as defined in Eq. (2.21), and the factor of $1 / 4$ accounts for universal KK wavefunction enhancements. For a chirality minus fermion, $\Psi_{-}$, one should simply make $\partial_{-} \leftrightarrow \partial_{+}$. If the zero-mode is right-handed, one should make $L \leftrightarrow R$ everywhere in Eq. (5.57).

As noted in subsection 5.1.4, the one-loop computation in $\xi=-3$ gauge automatically gives rise to operators with a gauge invariant structure, without any additional field redefinitions. In this gauge, we obtain

$$
\hat{r}_{\Psi}=-4 \times \frac{g_{4}^{2}}{16 \pi^{2}} C_{2}(\Psi) \Gamma\left(\frac{\epsilon}{2}\right), \quad c_{\Psi}=\frac{1}{2}
$$

and

$$
\hat{r}_{\Psi}^{\prime}=0
$$

so that no "mass-terms" are generated by the gauge interactions. A direct calculation of the three-point vertex of two fermions and a gauge field in the $\xi=-3$ gauge should give the correct coefficient to provide the gauge invariant completion of the kinetic term operators of Eq. (5.57). 


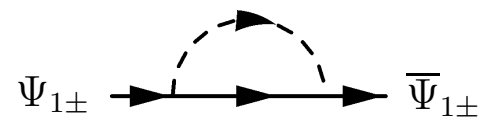

Figure 5: One-loop fermion self-energy diagrams associated with the Yukawa interaction (5.60). The dashed line denotes a complex scalar satisfying boundary conditions labeled by $n$.

Not all types of kinetic operators appear in Eq. (5.57). This is consistent with the fact that, when $n_{L}^{ \pm}=0$, so that there is a left-handed zero-mode, $\Psi_{ \pm R}$ vanishes at the fixed points $(0,0)$, $(L, L)$, and $(L, 0)$. Therefore, the quadratic operators not appearing in Eq. (5.57) vanish at the singular points [note also that $\Gamma^{ \pm} \Psi_{ \pm L}=\Gamma^{\mp} \Psi_{ \pm R}=0$, according to Eq. (5.47)].

\subsubsection{Yukawa Interactions}

We finally consider the effect of Yukawa couplings such as

$$
\mathcal{L}_{\text {Yukawa }}=\lambda_{6} \Phi \bar{\Psi}_{1 \pm} \Psi_{2 \mp}+\text { h.c. }
$$

Note that the fermions must be different since they must have opposite $6 \mathrm{D}$ chiralities in order for the above coupling to be Lorentz invariant. $\Phi$ is a complex $6 \mathrm{D}$ scalar. Assume that the boundary conditions for the scalar are labeled by $n$, while the boundary conditions for $\Psi_{1 \pm}$ and $\Psi_{2 \pm}$ are labeled by $n_{1 L}^{ \pm}, n_{1 R}^{ \pm}, n_{2 L}^{\mp}$ and $n_{2 R}^{\mp}$, such that, according to Eq. (3.2),

$$
\begin{aligned}
& n_{1 R}^{ \pm}=n_{1 L}^{ \pm} \mp 1 \bmod 4 \\
& n_{2 R}^{\mp}=n_{2 L}^{\mp} \pm 1 \bmod 4 .
\end{aligned}
$$

In addition, 6D Lorentz invariance of the local coupling (5.60) also requires [10]

$$
n-n_{1 L}^{ \pm}+n_{2 R}^{\mp}=0 \bmod 4
$$

which, together with (5.61) implies $n-n_{1 R}^{ \pm}+n_{2 L}^{\mp}=0 \bmod 4$. To be concrete, let us assume that $\Psi_{1 \pm}$ has a left-handed zero-mode: $n_{1 L}^{ \pm}=0$ and $n_{1 R}^{ \pm}=\mp 1$. Then Eqs. (5.61) and (5.62) imply

$$
n_{2 R}^{\mp}=-n \bmod 4, \quad n_{2 L}^{\mp}=-n \mp 1 \bmod 4 .
$$

The diagram in Figure 5 is then

$$
-\left(\frac{1}{4}\right)\left|\lambda_{4}\right|^{2} \sum_{m_{1}, l_{1}} \sum_{m_{1}^{\prime}, l_{1}^{\prime}} \int \frac{d^{D} k}{(2 \pi)^{D}} G_{k, n_{2}^{\mp}}^{\mp\left(m_{2}, l_{2} ; m_{2}^{\prime}, l_{2}^{\prime}\right)} G_{k+p, n}^{\left(m_{1}, l_{1} ; m_{1}^{\prime}, l_{1}^{\prime}\right)} P_{ \pm} .
$$

This contains a term that depends on the 4D momentum, with the structure

$$
k_{\lambda} \Gamma^{\lambda} \sum_{m_{1}, l_{1}} \sum_{m_{1}^{\prime}, l_{1}^{\prime}}\left[P_{R} \hat{\delta}\left(m_{2}, l_{2} ; m_{2}^{\prime}, l_{2}^{\prime} ; n_{2 L}^{\mp}\right)+P_{L} \hat{\delta}\left(m_{2}, l_{2} ; m_{2}^{\prime}, l_{2}^{\prime} ; n_{2 R}^{\mp}\right)\right] \hat{\delta}\left(m_{1}, l_{1} ; m_{1}^{\prime}, l_{1}^{\prime} ; n\right) P_{ \pm}
$$




\begin{tabular}{|c|c|c|c|c|c|c|c|c|}
\hline \multicolumn{4}{|c|}{$A_{\Psi}$} & \multicolumn{5}{|c|}{$B_{\Psi}$} \\
\hline & $n=0$ & $n=1,3$ & $n=2$ & & $n=0$ & $n=1$ & $n=2$ & $n=3$ \\
\hline \multirow{3}{*}{$\frac{1}{2} \times$} & 3 & -1 & -1 & & $3 / 2$ & $1 / 2$ & $-1 / 2$ & $-3 / 2$ \\
\hline & 2 & 0 & -2 & $1 \times$ & 1 & 1 & -1 & -1 \\
\hline & $5 / 2$ & $-1 / 2$ & $-3 / 2$ & & $5 / 4$ & $3 / 4$ & $-3 / 4$ & $-5 / 4$ \\
\hline
\end{tabular}

Table 4: Scalar functions $A_{\Psi}$ and $B_{\Psi}$ associated with the Yukawa contributions to the fermion selfenergies, as defined via Eq. (5.66), corresponding to the diagram of Fig. 同. We assume that the fermion has $6 \mathrm{D}$ chirality + , and that it has a zero-mode, i.e. we exclude $n_{L, R}^{+}=2$ boundary conditions. We give the results for scalars satisfying the four types of boundary conditions, labeled by $n=0,1,2,3$. For a given $n$ and for each diagram, there are three possible cases depending on KK-parity and whether $m-m^{\prime}$ is even or odd, as listed in Eq. (2.30): the first two cases correspond to even-even mixings with $m-m^{\prime}$ even and $m-m^{\prime}$ odd, and the third case to odd-odd mixings.

but given the relations (5.63), and the comments following Eq. (A.17), only the second term gives a nonvanishing result. The terms depending on momenta along the extra dimensions are both non-vanishing, and give rise to the following structure:

$$
\begin{gathered}
\sum_{m_{1}, l_{1}} \sum_{m_{1}^{\prime}, l_{1}^{\prime}} r_{m_{2}, \mp l_{2}} M_{m_{2}, l_{2}} \hat{\delta}\left(m_{2}, l_{2} ; m_{2}^{\prime}, l_{2}^{\prime} ; n_{2 L}^{\mp}\right) \hat{\delta}\left(m_{1}, l_{1} ; m_{1}^{\prime}, l_{1}^{\prime} ; n\right) \Gamma^{4} P_{R} P_{ \pm} \rightarrow r_{m^{\prime}, \mp l^{\prime}} M_{m^{\prime}, l^{\prime}} \Gamma^{4} P_{R} P_{ \pm} \\
\sum_{m_{1}, l_{1}} \sum_{m_{1}^{\prime}, l_{1}^{\prime}} r_{m_{2}, l_{2}} M_{m_{2}, l_{2}} \hat{\delta}\left(m_{2}, l_{2} ; m_{2}^{\prime}, l_{2}^{\prime} ; n_{2 R}^{\mp}\right) \hat{\delta}\left(m_{1}, l_{1} ; m_{1}^{\prime}, l_{1}^{\prime} ; n\right) \Gamma^{4} P_{L} P_{ \pm} \rightarrow r_{m, \pm l} M_{m, l} \Gamma^{4} P_{L} P_{ \pm} .
\end{gathered}
$$

With the help of Eq. (A.17) and the relations following it, we can write the diagrams in the form

$$
i \frac{\left|\lambda_{4}\right|^{2}}{16 \pi^{2}} \Gamma\left(\frac{\epsilon}{2}\right)\left\{A_{\Psi} p_{\mu} \Gamma^{\mu} P_{L}-B_{\Psi} \Gamma^{4}\left(r_{m^{\prime}, \mp l^{\prime}} M_{m^{\prime}, l^{\prime}} P_{R}+r_{m, \pm l} M_{m, l} P_{L}\right)\right\} P_{ \pm}
$$

where $A_{\psi}$ and $B_{\Psi}$ are given in Table 4 for a $6 \mathrm{D}$ chirality + fermion, and for the four allowed values of the boundary conditions satisfied by the scalar field, $n=0,1,2$ or 3 . For a $6 \mathrm{D}$ chirality - fermion, the cases $n=1$ and $n=3$ are interchanged. Also, when the zero mode of $\Psi_{1 \pm}$ is right-handed, we obtain Eq. (5.66) with $P_{L} \leftrightarrow P_{R}$.

The Yukawa interactions induce localized operators as in Eq. (5.57). When the scalar field satisfies $n=0$ boundary conditions we get $\hat{r}_{\Psi}=\hat{r}_{\Psi}^{\prime}, c_{\Psi}=c_{\Psi}^{\prime}$, and

$$
\hat{r}_{\Psi}=\frac{5}{8} \times \frac{\left|\lambda_{4}\right|^{2}}{16 \pi^{2}} \Gamma\left(\frac{\epsilon}{2}\right), \quad c_{\Psi}=\frac{2}{5} .
$$

The coefficients of the localized operators for other types of scalar boundary conditions can be easily worked out from Table 4 .

\subsection{Two-Point Functions of Scalar Fields}

In this section we study the new features arising in the calculation of the one-loop corrections to the two-point function associated with 6D scalars. Here we encounter the most general KKnumber violating structure arising from operators localized at the three conical singularities. In 


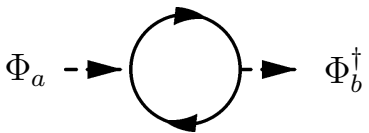

(a)

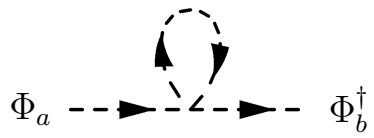

(d)

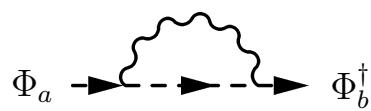

(b)

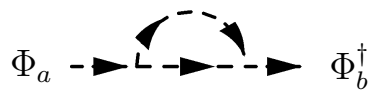

(e)

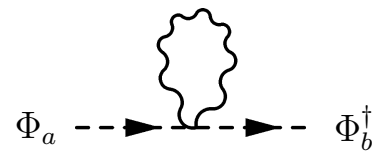

(c)

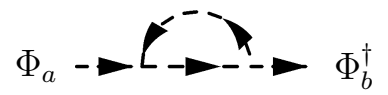

(f)

Figure 6: One-loop contribution to $\left\langle\Phi \Phi^{\dagger}\right\rangle$, where $\Phi$ is a complex scalar (represented by a dashed line with a single arrow) satisfying any of the four types of boundary conditions, $n=0,1,2,3$. The dashed lines with two arrows represent the propagation of $A_{+}$.

addition, this will serve as a warm up to study the renormalization associated with the spinless adjoints, $A_{ \pm}$.

As is well-known, these diagrams contain quadratic divergences that tend to lift the zeromode to the cutoff scale. This issue only affects scalars satisfying $n=0$ boundary conditions, and we assume that a bare contribution is tuned to keep the zero-mode light, if necessary. We are interested in the induced KK-number violating transitions that correspond to operators localized at the conical singularities. Notice that for $n=1,3$, the mass term operator

$$
-M^{2} \Phi^{\dagger} \Phi
$$

vanishes when evaluated at the singular points $(0,0),(L, L)$ and $(0, L)$, due to the vanishing of the corresponding KK wavefunctions given in Eq. (2.2). This is relevant for the spinless adjoints, $A_{ \pm}$, which satisfy these boundary conditions, and it trivially shows that there are no divergences corresponding to localized mass terms for these fields. For $n=2$ boundary conditions, the mass term operator (5.68) is non-vanishing at $(0, L)$.

Here we will simply use dimensional regularization to calculate the induced localized kinetic term operators, and will not worry about potential quadratic divergences. We show the relevant diagrams in Figure 6 .

\subsubsection{Gauge Interactions}

Let us consider first the diagrams arising from the gauge interactions. The interactions with the spin- 1 components of the $6 \mathrm{D}$ gauge field have expressions which are straightforward generalizations of those applying to 4D scalars minimally coupled to gauge fields (see Figure 2). The coupling between two scalars and one gauge field induces

$$
(b)=-\left(\frac{1}{4}\right) g_{4}^{2} C_{2}(\Phi) \delta_{a b} \sum_{m_{1}, l_{1}} \sum_{m_{1}^{\prime}, l_{1}^{\prime}} \int \frac{d^{D} k}{(2 \pi)^{D}}(2 p-k)^{\mu}(2 p-k)^{\nu} G_{\mu \nu, k}^{\left(m_{1}, l_{1} ; m_{1}^{\prime}, l_{1}^{\prime}\right)} G_{p-k, n}^{\left(m_{2}, l_{2} ; m_{2}^{\prime}, l_{2}^{\prime}\right)},
$$


where $\left(T^{c} T^{c}\right)_{a b}=C_{2}(\Phi) \delta_{a b}$ is the quadratic Casimir operator in the representation of $\Phi$. The coupling with two gauge bosons induces

$$
(c)=i g_{4}^{2} C_{2}(\Phi) \delta_{a b} \sum_{m_{1}, l_{1}} \int \frac{d^{D} k}{(2 \pi)^{D}} \eta^{\mu \nu} G_{\mu \nu, k}^{\left(m_{1}, l_{1} ; m_{1}^{\prime}, l_{1}^{\prime}\right)},
$$

which, as explained in subsection 5.1.1 should be contracted with external propagators to obtain the correct KK-number violating structure.

In addition, there are diagrams arising from the gauge couplings to the spin- 0 components of the 6D gauge field. The corresponding vertices are shown in Figure 1. A quartic interaction induces

$$
(d)=-i g_{4}^{2} C_{2}(\Phi) \delta_{a b} \sum_{m_{1}, l_{1}} \int \frac{d^{D} k}{(2 \pi)^{D}} G_{++, k}^{\left(m_{1}, l_{1} ; m_{1}^{\prime}, l_{1}^{\prime}\right)}
$$

which again requires contraction with external propagators, while a trilinear interaction gives

$$
\begin{aligned}
(e)=-\left(\frac{1}{4}\right)^{2} g_{4}^{2} C_{2}(\Phi) & \delta_{a b} \sum_{m_{1}, l_{1}} \sum_{m_{1}^{\prime}, l_{1}^{\prime}} \int \frac{d^{D} k}{(2 \pi)^{D}}\left[r_{m, l}^{*} M_{m, l}+r_{m_{2}, l_{2}}^{*} M_{m_{2}, l_{2}}\right] \\
& \times G_{++, p-k}^{\left(m_{1}, l_{1} ; m_{1}^{\prime}, l_{1}^{\prime}\right)}\left[r_{m_{2}^{\prime}, l_{2}^{\prime}} M_{m_{2}^{\prime}, l_{2}^{\prime}}+r_{m^{\prime}, l^{\prime}} M_{m^{\prime}, l^{\prime}}\right] G_{k, n}^{\left(m_{2}, l_{2} ; m_{2}^{\prime}, l_{2}^{\prime}\right)},
\end{aligned}
$$

and a second diagram $(f)$ which is obtained from $(e)$ by taking the complex conjugates of all phases, $r_{m, l} \rightarrow r_{m, l}^{*}$, and also $G_{++} \rightarrow G_{--}$.

We wish to consider scalar fields satisfying any of the four possible boundary conditions, $n=0,1,2$ or 3 . We start by considering the cases $n=0$ or $n=2$. We write the results for diagrams $(b)-(f)$ in the form

$$
i \frac{g_{4}^{2}}{16 \pi^{2}} C_{2}(\Phi) \delta_{a b} \Gamma\left(\frac{\epsilon}{2}\right)\left\{A_{\Phi} p^{2}-B_{\Phi}\left(M_{m, l}^{2}+M_{m^{\prime}, l^{\prime}}^{2}\right)\right\}
$$
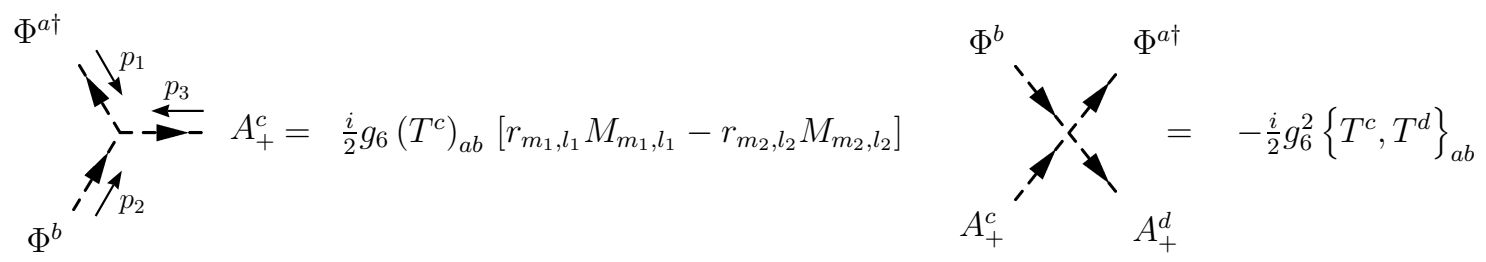

Figure 7: Momentum space Feynman rules for the couplings between scalars and the spinless adjoints, $A_{ \pm}$. There is a trilinear vertex involving an incoming $A_{+}$(outgoing $A_{-}$), which can be obtained from the one shown here by $r_{m, l} \rightarrow r_{m, l}^{*}$, where the momentum-dependent phases were defined in Eq. (2.27). 


\begin{tabular}{|c|c|c|c|c|c|c|}
\hline & \multicolumn{3}{|c|}{$A_{\Phi}$} & \multicolumn{3}{|c|}{$B_{\Phi}$} \\
\hline & & $n=0$ & $n=2$ & & $n=0$ & $n=2$ \\
\hline (b) & $(\xi-3) \times\{$ & $\begin{array}{c}3 \\
2 \\
5 / 2\end{array}$ & $\begin{array}{c}0 \\
1 / 2 \\
-1 / 2\end{array}$ & $\left(\xi+\xi^{2}\right) \times\{$ & $\begin{array}{c}5 / 4 \\
1 \\
9 / 8\end{array}$ & $\begin{array}{c}0 \\
1 / 8 \\
-1 / 8\end{array}$ \\
\hline (c) & & 0 & 0 & $-\left(3+\xi^{2}\right) \times$ & $\begin{array}{c}5 / 4 \\
1 \\
9 / 8\end{array}$ & $\begin{array}{c}0 \\
1 / 8 \\
-1 / 8\end{array}$ \\
\hline (d) & & 0 & 0 & $-(1+\xi) \times\{$ & $\begin{array}{c}-1 / 4 \\
0 \\
-1 / 8\end{array}$ & $\begin{array}{c}0 \\
-1 / 8 \\
1 / 8\end{array}$ \\
\hline$(\mathrm{e})+(\mathrm{f})$ & & 0 & 0 & $-\frac{1}{2} \times\{$ & $\begin{array}{c}-7 / 2 \\
-2 \\
-11 / 4\end{array}$ & $\begin{array}{c}0 \\
-3 / 4 \\
3 / 4\end{array}$ \\
\hline
\end{tabular}

Table 5: Functions $A_{\Phi}$ and $B_{\Phi}$, as defined via Eq. (5.73), associated with the gauge contributions to the scalar self-energies, corresponding to diagrams $(b)-(f)$ in Fig. 6. We give the results for scalars satisfying $n=0$ and $n=2$ boundary conditions. For $n=0$, the three cases in each diagram correspond to cases 1a, $1 \mathrm{~b}$ and 2 of Eq. (2.30), while for $n=2$, they correspond to cases 1, 2a and 2b of Eq. (2.32).

A straightforward calculation gives the scalar coefficients $A_{\Phi}$ and $B_{\Phi}$ as summarized in Table 5 . Adding the diagrams for the $n=0$ boundary conditions we get

$$
A_{\Phi}=(\xi-3) \times\left\{\begin{array}{c}
3 \\
2 \\
5 / 2
\end{array}, \quad B_{\Phi}=\xi \times\left\{\begin{array}{l}
3 / 2 \\
1 \\
5 / 4
\end{array}-\left\{\begin{array}{l}
7 / 4 \\
2 \\
15 / 8
\end{array}\right.\right.\right.
$$

where the three cases correspond to those listed in Eq. (2.30).

For scalars satisfying $n=2$ boundary conditions we get instead

$$
A_{\Phi}=(\xi-3) \times\left\{\begin{array}{r}
0 \\
1 / 2 \\
-1 / 2
\end{array}, \quad B_{\Phi}=\xi \times\left\{\begin{array}{r}
0 \\
1 / 4 \\
-1 / 4
\end{array}+\left\{\begin{array}{r}
0 \\
1 / 8 \\
-1 / 8
\end{array}\right.\right.\right.
$$

where now the three cases correspond to those listed in Eq. (2.32). Notice that, to lowest order, the corresponding mass shifts are proportional to $2 B_{\Phi}-A_{\Phi}$, and that the $\xi$ dependence cancels out in the difference, both in Eqs. (5.74) and (5.75).

It remains to consider the gauge contributions to scalars satisfying $n=1$ or $n=3$ boundary conditions. In these cases we write the results for diagrams $(b)-(f)$ in the form

$$
-i \frac{g_{4}^{2}}{16 \pi^{2}} C_{2}(\Phi) \delta_{a b} \Gamma\left(\frac{\epsilon}{2}\right) M_{m, l} M_{m^{\prime}, l^{\prime}}\left\{B_{\Phi} r_{m, l} r_{m^{\prime}, l^{\prime}}^{*}+B_{\Phi}^{\prime} r_{m, l}^{*} r_{m^{\prime}, l^{\prime}}\right\}
$$




\begin{tabular}{|c|c|c|c|c|c|}
\hline & \multicolumn{2}{|l|}{$B_{\Phi}$} & \multicolumn{3}{|l|}{$B_{\Phi}^{\prime}$} \\
\hline (b) & $\xi \times\left\{\begin{array}{l}1 / 4 \\
0 \\
1 / 8 \\
1 / 8\end{array}-\xi\right.$ & $\times\left\{\begin{array}{l}5 / 4 \\
1 \\
9 / 8 \\
9 / 8\end{array}\right.$ & $\xi \times\left\{\begin{array}{l}0 \\
0 \\
1 / 8 \\
-1 / 8\end{array}-\xi\right.$ & $\times\{$ & $\begin{array}{l}0 \\
0 \\
1 / 8 \\
-1 / 8\end{array}$ \\
\hline (c) & $\left(3+\xi^{2}\right) \times$ & $\begin{array}{l}5 / 4 \\
1 \\
9 / 8 \\
9 / 8\end{array}$ & $\left(3+\xi^{2}\right) \times$ & $\begin{array}{l}0 \\
0 \\
1 / 8 \\
-1 /\end{array}$ & \\
\hline (d) & $-(1+\xi) \times$ & $\begin{array}{l}1 / 4 \\
0 \\
1 / 8 \\
1 / 8\end{array}$ & $-(1+\xi) \times$ & $\begin{array}{l}0 \\
0 \\
1 / 8 \\
-1\end{array}$ & \\
\hline$(\mathrm{e})+(\mathrm{f})$ & $-\frac{1}{2} \times\left\{\begin{array}{l}2 \\
3\end{array}\right.$ & $\begin{array}{l}/ 2 \\
4 \\
4\end{array}$ & $-\frac{1}{2} \times\{$ & $\begin{array}{l} \\
-5 / 4 \\
/ 4\end{array}$ & \\
\hline
\end{tabular}

Table 6: Functions $B_{\Phi}$ and $B_{\Phi}^{\prime}$, as defined via Eq. (5.76), associated with the gauge contributions to the scalar self-energies, corresponding to diagrams $(b)-(f)$ in Fig. 6, in the case where the scalars satisfy $n=3$ boundary conditions. The four lines in each diagram correspond to cases $1 \mathrm{a}, 1 \mathrm{~b}, 2 \mathrm{a}$ and $2 \mathrm{~b}$ of Eqs. (2.30) and (2.32). For $n=1$, the roles of $B_{\Phi}$ and $B_{\Phi}^{\prime}$ are interchanged.

Notice that there is no $p^{2}$ term, as expected from the fact that the 4D-like kinetic operator for fields satisfying these boundary conditions vanishes at the conical singularities. A straightforward calculation gives the scalar coefficients $B_{\Phi}$ and $B_{\Phi}^{\prime}$ as summarized in Table 6 for the $n=3$ case. Adding the various contributions, we obtain

$$
B_{\Phi}=\left\{\begin{array}{c}
15 / 4 \\
2 \\
23 / 8 \\
23 / 8
\end{array}, \quad B_{\Phi}^{\prime}=\left\{\begin{array}{c}
0 \\
0 \\
7 / 8 \\
-7 / 8
\end{array}\right.\right.
$$

where the four lines correspond to cases 1a, 1b, 2a and 2b in Eqs. (2.30) and (2.32). We see that the $\xi$-dependence cancels out.

For $n=1$ boundary conditions, the roles of $B_{\Phi}$ and $B_{\Phi}^{\prime}$ in Eq. (5.77) are interchanged.

\subsubsection{Localized Operators}

Comparing to the general results of subsection 2.3, we see that the above expressions correspond to localized operators as in Eq. (2.22). Here we summarize the induced localized operators in terms of the coefficients $r_{i}=\hat{r}_{i} L^{2}, c_{i}, r_{3}^{\prime}=\hat{r}_{3}^{\prime} L^{2}$ and $c_{3}^{\prime}$ of Eq. (2.22), for the four possible boundary conditions defining the scalar field: 
- For $n=0$ :

$$
\begin{array}{ll}
\hat{r}_{1}=\frac{5}{4}(\xi-3) \times \frac{g_{4}^{2}}{16 \pi^{2}} C_{2}(\Phi) \Gamma\left(\frac{\epsilon}{2}\right), & c_{1}=\frac{2}{5}, \\
\hat{r}_{2}=\frac{5}{16}(2 \xi-3) \times \frac{g_{4}^{2}}{16 \pi^{2}} C_{2}(\Phi) \Gamma\left(\frac{\epsilon}{2}\right), & c_{2}=\frac{2}{5} \frac{(2 \xi+1)}{(2 \xi-3)},
\end{array}
$$

while $\hat{r}_{3}=\hat{r}_{3}^{\prime}=0$.

- For $n=2$ :

$$
\begin{aligned}
& c_{1} \hat{r}_{1}=\frac{1}{2}(\xi-3) \times \frac{g_{4}^{2}}{16 \pi^{2}} C_{2}(\Phi) \Gamma\left(\frac{\epsilon}{2}\right), \\
& c_{2} \hat{r}_{2}=\frac{1}{8}(2 \xi+1) \times \frac{g_{4}^{2}}{16 \pi^{2}} C_{2}(\Phi) \Gamma\left(\frac{\epsilon}{2}\right),
\end{aligned}
$$

while $\hat{r}_{3}=\hat{r}_{3}^{\prime}=0$. In this case, no operators at $(0,0)$ or $(L, L)$ are generated.

- For $n=3$ :

$$
\begin{array}{rlrl}
\hat{r}_{3} & =\frac{23}{16} \times \frac{g_{4}^{2}}{16 \pi^{2}} C_{2}(\Phi) \Gamma\left(\frac{\epsilon}{2}\right), \quad \quad c_{3}=\frac{14}{23}, \\
c_{3}^{\prime} \hat{r}_{3}^{\prime} & =\frac{7}{8} \times \frac{g_{4}^{2}}{16 \pi^{2}} C_{2}(\Phi) \Gamma\left(\frac{\epsilon}{2}\right), &
\end{array}
$$

while $\hat{r}_{1}=\hat{r}_{2}=0$. Notice that the operators associated with $r_{3}^{\prime}$ are generated only at $(0, L)$, not at $(0,0)$ or $(L, L)$.

- For $n=1$ :

$$
\begin{aligned}
c_{3} \hat{r}_{3} & =\frac{7}{8} \times \frac{g_{4}^{2}}{16 \pi^{2}} C_{2}(\Phi) \Gamma\left(\frac{\epsilon}{2}\right), \\
\hat{r}_{3}^{\prime} & =\frac{23}{16} \times \frac{g_{4}^{2}}{16 \pi^{2}} C_{2}(\Phi) \Gamma\left(\frac{\epsilon}{2}\right), \quad c_{3}^{\prime}=\frac{14}{23},
\end{aligned}
$$

while $\hat{r}_{1}=\hat{r}_{2}=0$. Notice that the operators associated with $r_{3}$ are generated only at $(0, L)$, not at $(0,0)$ or $(L, L)$.

\subsubsection{Yukawa Interactions}

We end this section by considering the effects of Yukawa interactions, which as explained in Eq. (5.60), involve two 6D Weyl fermions of opposite 6D chiralities. We have

$$
(a)=\left(\frac{1}{4}\right)\left|\lambda_{4}\right|^{2} \delta_{a b} \sum_{m_{1}, l_{1}} \sum_{m_{1}^{\prime}, l_{1}^{\prime}} \int \frac{d^{D} k}{(2 \pi)^{D}} \operatorname{Tr}\left\{G_{k+p}^{ \pm,\left(m_{1}, l_{1} ; m_{1}^{\prime}, l_{1}^{\prime}\right)} G_{k}^{\mp,\left(m_{2}^{\prime}, l_{2}^{\prime} ; m_{2}, l_{2}\right)} P_{\mp}\right\},
$$

which can be easily evaluated using the identities (5.16) and (5.47)-(5.49) to simplify the trace.

We restrict to the case where at least one of the $6 \mathrm{D}$ fermions running in the loop has a zero-mode. If the zero-mode is left-handed the boundary conditions for the fermions in the loop 
are related to those of the scalar by Eqs. (5.63). Analogous relations hold if the zero-mode is right-handed. The boundary conditions obeyed by the scalar field are labeled by $n$.

Independently of the chirality of the fermion zero-mode, when $n=0$ or $n=2$ we can write the result of the diagram as

$$
i \frac{\left|\lambda_{4}\right|^{2}}{16 \pi^{2}} \delta_{a b} \Gamma\left(\frac{\epsilon}{2}\right)\left\{A_{\Phi} p^{2}-B_{\Phi}\left(M_{m, l}^{2}+M_{m^{\prime}, l^{\prime}}^{2}\right)\right\}
$$

where the scalar coefficients $A_{\Phi}$ and $B_{\Phi}$ are given by

$$
\begin{array}{lll}
A_{\Phi}=2, & B_{\Phi}=2, & \text { for } n=0, \\
A_{\Phi}=0, & B_{\Phi}=0, & \text { for } n=2 .
\end{array}
$$

Notice that for $n=0$, this corresponds to localized operators as in Eq. (2.22), with

$$
\hat{r}_{1}=\hat{r}_{2}=\frac{g_{4}^{2}}{16 \pi^{2}} C_{2}(\Phi) \Gamma\left(\frac{\epsilon}{2}\right), \quad c_{1}=c_{2}=0
$$

and $\hat{r}_{3}=\hat{r}_{3}^{\prime}=0$.

When $n=1$ or $n=3$ the result depends on whether both $6 \mathrm{D}$ fermions in the loop give rise to a $4 \mathrm{D}$ chiral zero-mode, or only one of them. We write the results in the form

$$
-i \frac{\left|\lambda_{4}\right|^{2}}{16 \pi^{2}} \delta_{a b} \Gamma\left(\frac{\epsilon}{2}\right) M_{m, l} M_{m^{\prime}, l^{\prime}}\left\{B_{\Phi} r_{m, l} r_{m^{\prime}, l^{\prime}}^{*}+B_{\Phi}^{\prime} r_{m, l}^{*} r_{m^{\prime}, l^{\prime}}\right\} .
$$

Let us label the boundary conditions associated with the two fermions participating in the Yukawa interaction, Eq. (5.60), by $n_{i L}^{ \pm}$and $n_{i R}^{\mp}$, with $i=1,2$, as in subsection 5.2.3. For $n=3$, both fermions in the loop have a zero mode when either $n_{1 L}^{+}=n_{2 L}^{-}=0$ or $n_{1 R}^{-}=n_{2 R}^{+}=0$. In these instances, we get

$$
B_{\Phi}=\left\{\begin{array}{c}
1 \\
0 \\
1 / 2 \\
1 / 2
\end{array} \quad, \quad B_{\Phi}^{\prime}=\left\{\begin{array}{c}
0 \\
0 \\
-1 / 2 \\
1 / 2
\end{array}, \quad \text { for } n=3\right. \text { and two zero modes }\right.
$$

where the four lines correspond to cases 1a, 1b, 2a and 2b in Eqs. (2.30) and (2.32). For $n=1$, the two fermions in the loop can have zero-modes simultaneously when $n_{1 R}^{+}=n_{2 R}^{-}=0$ or $n_{1 L}^{-}=n_{2 L}^{+}=0$. In such instances, one obtains Eq. (5.87) with $B_{\Phi}$ and $B_{\Phi}^{\prime}$ interchanged.

The second category occurs when only one of the fermions has a zero-mode. For $n=3$, this happens when $n_{1 R}^{+}=0$ or $n_{1 L}^{-}=0$ or $n_{2 L}^{+}=0$ or $n_{2 R}^{-}=0$, while for $n=1$, it happens when $n_{1 L}^{+}=0$ or $n_{1 R}^{-}=0$ or $n_{2 R}^{+}=0$ or $n_{2 L}^{-}=0$. In all such instances, one gets the opposite sign of the contributions when two fermion zero-modes are simultaneously present.

\subsection{Two-Point Function of the Spinless Adjoints}

We end our exploration of the one loop corrections in the chiral square background by studying the two-point functions associated with the spinless adjoints, $A_{ \pm}$. 


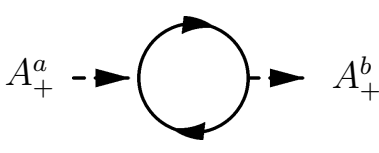

(a)

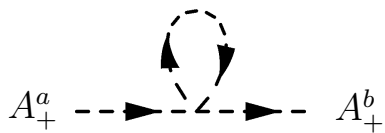

(d)

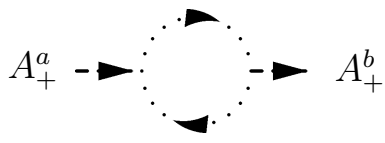

(g)

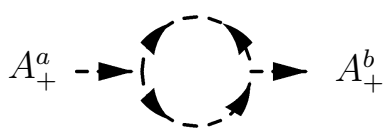

(j)

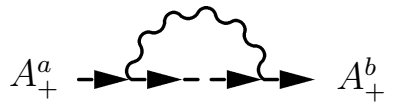

(b)

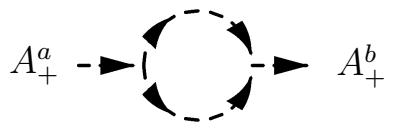

(e)

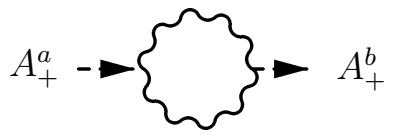

(h)

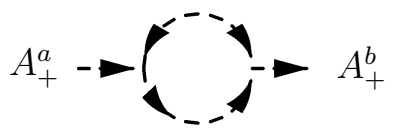

$(\mathrm{k})$

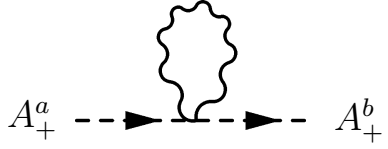

(c)

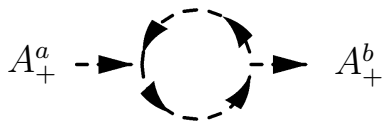

(f)

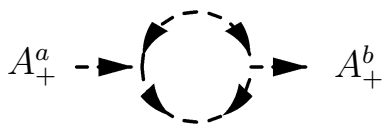

(i)

Figure 8: One-loop contribution to $\left\langle A_{+} A_{+}^{\dagger}\right\rangle$. The dashed lines with two arrows represent the propagation of $A_{+}$.

We need to consider two types of two-point functions: $\left\langle A_{+} A_{+}^{\dagger}\right\rangle$ and $\left\langle A_{+} A_{+}\right\rangle$, which together with their complex conjugates determine the structure of localized counterterms needed to absorb the logarithmic divergences that appear at one-loop. As mentioned in the previous section, the fact that the spinless adjoints satisfy $n=1$ or $n=3$ boundary conditions ensures that no localized mass terms are generated. However, one can generate a localized tadpole term, proportional to $F_{45}$, the field strength with indices in the extra dimensions 12]. The coefficient is quadratically divergent, but is proportional to the trace of the generators associated with the field in the loop. Since for the standard model gauge group and field content all such traces vanish, we do not compute this tadpole in what follows. It is easy to do with the technology we have developed.

\subsubsection{Gauge Interactions}

We show the diagrams contributing to the two point function $\left\langle A_{+} A_{+}^{\dagger}\right\rangle$ in Figure 8 , and those contributing to $\left\langle A_{+} A_{+}\right\rangle$in Figure 9. We start with the diagrams in Figure 8.

Diagrams $(b)-(d)$ are trivially related to the corresponding diagrams encountered in our study of general scalar fields in section 5.3. Diagram $(b)$ in Figure 8 is given by $(1 / 2)^{2}$ times 
expression (5.69) with $G_{n} \rightarrow G_{++}$, and $C_{2}(\Phi) \rightarrow C_{2}(A)$. Similarly, diagram $(c)$ is given by $1 / 2$ times expression (5.70) with $C_{2}(\Phi) \rightarrow C_{2}(A)$. Diagram $(d)$ is given by $1 / 4$ of expression (5.71). Diagrams $(e)$ and $(f)$ are given by

$$
\begin{aligned}
(e)= & -\left(\frac{1}{4}\right)^{3} \frac{g_{4}^{2}}{2} C_{2}(A) \delta_{a b} \sum_{m_{1}, l_{1}} \sum_{m_{1}^{\prime}, l_{1}^{\prime}} \int \frac{d^{D} k}{(2 \pi)^{D}}\left[r_{m_{1}, l_{1}}^{*} M_{m_{1}, l_{1}}+r_{m_{2}, l_{2}}^{*} M_{m_{2}, l_{2}}\right] \\
& \times G_{++, k+p}^{\left(m_{1}, l_{1} ; m_{1}^{\prime}, l_{1}^{\prime}\right)}\left[r_{m_{1}^{\prime}, l_{1}^{\prime}} M_{m_{1}^{\prime}, l_{1}^{\prime}}+r_{m_{2}^{\prime}, l_{2}^{\prime}} M_{m_{2}^{\prime}, l_{2}^{\prime}}\right] G_{++, k}^{\left(m_{2}, l_{2} ; m_{2}^{\prime}, l_{2}^{\prime}\right)}, \\
(f)= & -\left(\frac{1}{4}\right)^{3} g_{4}^{2} C_{2}(A) \delta_{a b} \sum_{m_{1}, l_{1}} \sum_{m_{1}^{\prime}, l_{1}^{\prime}} \int \frac{d^{D} k}{(2 \pi)^{D}}\left[r_{m, l} M_{m, l}+r_{m_{1}, l_{1}} M_{m_{1}, l_{1}}\right] \\
& \times G_{--, k+p}^{\left(m_{1}, l_{1} ; m_{1}^{\prime}, l_{1}^{\prime}\right)}\left[r_{m^{\prime}, l^{\prime}}^{*} M_{m^{\prime}, l^{\prime}}+r_{m_{1}^{\prime}, l_{1}^{\prime}}^{*} M_{m_{1}^{\prime}, l_{1}^{\prime}}\right] G_{++, k}^{\left(m_{2}, l_{2} ; m_{2}^{\prime}, l_{2}^{\prime}\right)} .
\end{aligned}
$$

Finally, there is a diagram involving the ghost fields,

$$
\begin{aligned}
(g)=\left(\frac{1}{4}\right)^{2} \xi^{2} g_{4}^{2} C_{2}(A) \delta_{a b} \sum_{m_{1}, l_{1}} \sum_{m_{1}^{\prime}, l_{1}^{\prime}} & r_{m_{2}, l_{2}} M_{m_{2}, l_{2}} r_{m_{1}^{\prime}, l_{1}^{\prime}}^{*} M_{m_{1}^{\prime}, l_{1}^{\prime}} \\
& \times \int \frac{d^{D} k}{(2 \pi)^{D}} G_{\xi, k+p}^{\left(m_{1}, l_{1} ; m_{1}^{\prime}, l_{1}^{\prime}\right)} G_{\xi, k}^{\left(m_{2}^{\prime}, l_{2}^{\prime} ; m_{2}, l_{2}\right)},
\end{aligned}
$$

and one involving the interaction between two gauge fields and a single spinless adjoint given by

$$
\begin{aligned}
&(h)=-\left(\frac{1}{4}\right)^{2} \frac{g_{4}^{2}}{2} C_{2}(A) \delta_{a b} \sum_{m_{1}, l_{1}} \sum_{m_{1}^{\prime}, l_{1}^{\prime}} \int \frac{d^{D} k}{(2 \pi)^{D}}\left[r_{m_{1}, l_{1}} M_{m_{1}, l_{1}}+r_{m_{2}, l_{2}} M_{m_{2}, l_{2}}\right] \\
& \times G_{k+p}^{\mu \nu\left(m_{1}, l_{1} ; m_{1}^{\prime}, l_{1}^{\prime}\right)}\left[r_{m_{1}^{\prime}, l_{1}^{\prime}}^{*} M_{m_{1}^{\prime}, l_{1}^{\prime}}+r_{m_{2}^{\prime}, l_{2}^{*}}^{*} M_{m_{2}^{\prime}, l_{2}^{\prime}}\right] G_{\mu \nu, k}^{\left(m_{2}^{\prime}, l_{2}^{\prime} ; m_{2}, l_{2}\right)} .
\end{aligned}
$$

These last two diagrams are present to account for the would-be Goldstone modes contained in $A_{+}$that realize the Higgs mechanism at each spin-1 KK level.

Notice that diagrams $(i),(j)$ and $(k)$ in Figure 8, involving the propagators $G_{+-}$or $G_{-+}$, are finite as a result of a cancellation between the two real degrees of freedom in $A_{+}$, so we need not consider them.

We write the results for diagrams $(b)-(h)$ in the form

$$
-i \frac{g_{4}^{2}}{16 \pi^{2}} C_{2}(A) \delta_{a b} \Gamma\left(\frac{\epsilon}{2}\right) M_{m, l} M_{m^{\prime}, l^{\prime}}\left\{B_{+} r_{m, l} r_{m^{\prime}, l^{\prime}}^{*}+B_{+}^{\prime} r_{m, l}^{*} r_{m^{\prime}, l^{\prime}}\right\} .
$$

Notice that there is no $p^{2}$ term, as expected from the fact that the $4 \mathrm{D}$ kinetic operator for fields satisfying $n=3$ boundary conditions vanishes at the conical singularities. A straightforward calculation gives the scalar coefficients $B_{+}$and $B_{+}^{\prime}$ as summarized in Table 7 . Adding the various contributions, we obtain

$$
B_{+}=\left\{\begin{array}{c}
9 / 2 \\
7 / 2 \\
4
\end{array} \quad B_{+}^{\prime}=0\right.
$$




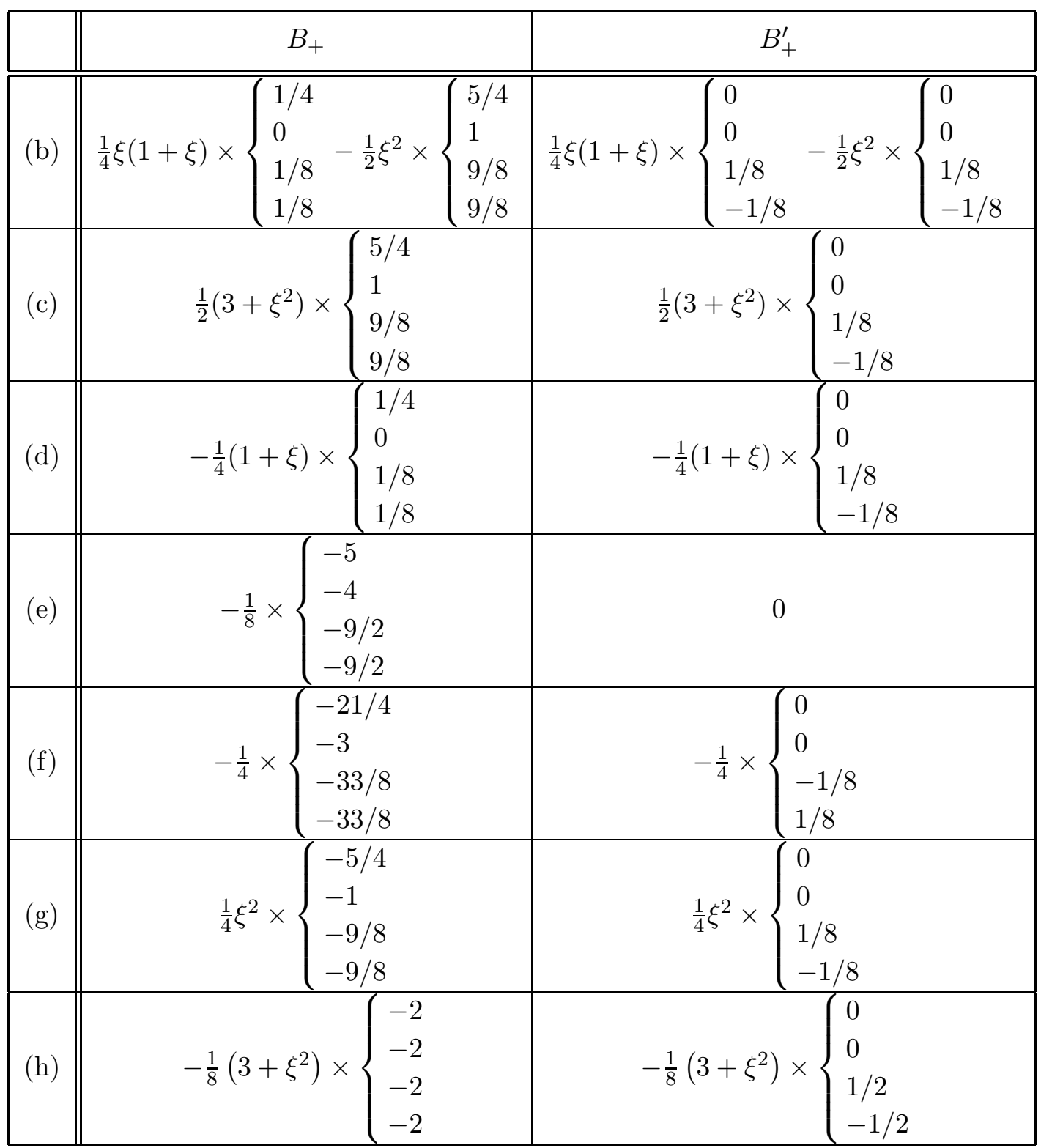

Table 7: Functions $B_{+}$and $B_{+}^{\prime}$, as defined via Eq. (5.92), associated with the gauge contributions to the two-point function $\left\langle A_{+} A_{+}^{\dagger}\right\rangle$, corresponding to diagrams $(b)-(h)$ in Fig. 8. The four lines in each diagram correspond to cases 1a, 1b, 2a and 2b of Eqs. (2.30) and (2.32).

where the three lines correspond to the cases listed in Eq. 2.30). We see that the $\xi$-dependence disappears and that $B_{+}^{\prime}$ vanishes. Notice that the vanishing of $B_{+}^{\prime}$ is essential to get a rotationally invariant (in the plane of the extra dimensions) structure for the induced localized operators: a nonvanishing result would lead to operators of the form $\left(\partial_{+} A_{+}\right)\left(\partial_{-} A_{-}\right) . B_{+}$, on the other hand, leads to localized operators of the form $\left(\partial_{-} A_{+}\right)\left(\partial_{+} A_{-}\right)$, which are rotationally invariant.

Consider now the diagrams in Figure 9 for the $\left\langle A_{+} A_{+}\right\rangle$two-point function. Diagram $(m)$ can 


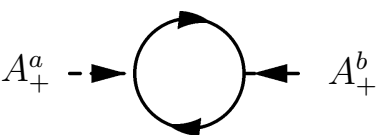

(l)

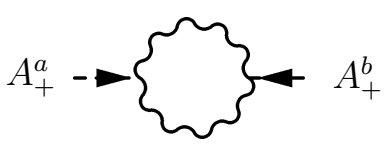

(o)

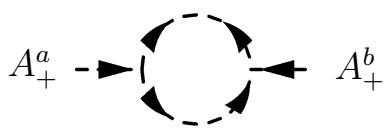

$(\mathrm{r})$

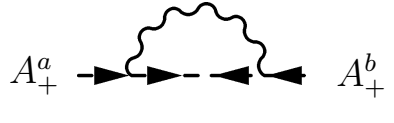

$(\mathrm{m})$

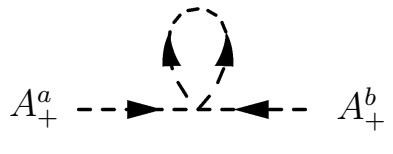

(p)

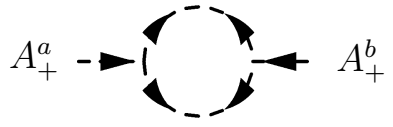

$(\mathrm{s})$

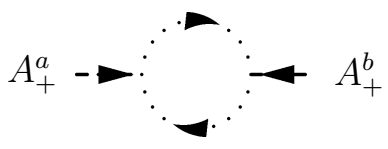

(n)

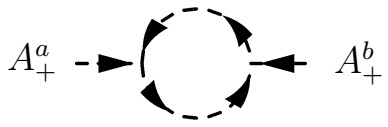

(q)

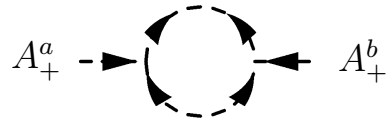

$(\mathrm{t})$

Figure 9: One-loop contribution to $\left\langle A_{+} A_{+}\right\rangle$. The dashed lines with two arrows represent the propagation of $A_{+}$.

be obtained from diagram (b) in Figure 8 , with the replacement $G_{++} \rightarrow-G_{-+}$, the minus sign coming from the ordering of the $4 \mathrm{D}$ momenta. Diagram $(n)$ can be obtained from Eq. (5.90) with $r_{m_{2}, l_{2}} \rightarrow r_{m_{2}, l_{2}}^{*}$. Similarly, diagram (o) can be obtained from Eq. (5.91) by making the replacements $r_{m_{1}, l_{1}} \rightarrow r_{m_{1}, l_{1}}^{*}$ and $r_{m_{2}, l_{2}} \rightarrow r_{m_{2}, l_{2}}^{*}$. Diagram $(p)$ can be obtained from diagram $(d)$ in Figure 8, with the replacement $G_{++} \rightarrow G_{-+}$. Finally, diagram $(q)$ can be obtained from Eq. (5.89) by making the replacements $r_{m, l} \rightarrow-r_{m, l}^{*}$ and $r_{m_{1}, l_{1}} \rightarrow r_{m_{2}, l_{2}}^{*}$. It is easy to see that diagrams $(r),(s)$ and $(t)$ in Figure 9 are finite as a result of a cancellation between the two real scalar degrees of freedom in $A_{+}$, and we do not consider them in the following.

We write the results for diagrams $(m)-(q)$ in the form

$$
-i \frac{g_{4}^{2}}{16 \pi^{2}} C_{2}(A) \delta_{a b} \Gamma\left(\frac{\epsilon}{2}\right) r_{m, l}^{*} r_{m^{\prime}, l^{\prime}}^{*} M_{m, l} M_{m^{\prime}, l^{\prime}} \tilde{B}_{+} .
$$

A straightforward calculation gives the scalar coefficients $\tilde{B}_{+}$as summarized in Table 8. Adding the various contributions, we obtain

$$
\tilde{B}_{+}=\left\{\begin{array}{c}
-9 / 2 \\
-7 / 2 \\
-4
\end{array},\right.
$$

where the three lines correspond to the cases listed in Eq. (2.30). Again, the $\xi$ dependence cancels out, as it should. Furthermore, we see from Eqs. (5.93) and (5.95) that $\tilde{B}_{+}=-B_{+}$, which is essential to obtain a gauge invariant structure for the induced localized operators, as we discuss in subsection 5.4.4. 


\begin{tabular}{|c||c|}
\hline & $\tilde{B}_{+}$ \\
\hline \hline$(\mathrm{m})$ & $-\frac{1}{4} \xi(1-\xi) \times\left\{\begin{array}{l}5 / 2 \\
2 \\
9 / 4\end{array}\right.$ \\
\hline$(\mathrm{p})$ & $-\frac{1}{4}(1-\xi) \times\left\{\begin{array}{l}5 / 2 \\
2 \\
9 / 4\end{array}\right.$ \\
\hline
\end{tabular}

\begin{tabular}{|c||c|}
\hline & $\tilde{B}_{+}$ \\
\hline \hline \multirow{2}{*}{ (n) } & $\frac{1}{4} \xi^{2} \times\left\{\begin{array}{l}1 \\
1 \\
1\end{array}\right.$ \\
\hline (q) & $\frac{1}{4} \times\left\{\begin{array}{l}-5 \\
-3 \\
-4\end{array}\right.$ \\
\hline
\end{tabular}

\begin{tabular}{|c||c|}
\hline & $\tilde{B}_{+}$ \\
\hline \hline \multirow{2}{*}{ (o) } & $-\frac{1}{8}\left(3+\xi^{2}\right) \times\left\{\begin{array}{l}7 \\
6 \\
13 / 2\end{array}\right.$ \\
\hline
\end{tabular}

Table 8: Scalar functions $\tilde{B}_{+}$, as defined via Eq. (5.94), associated with the gauge contributions to the two-point function $\left\langle A_{+} A_{+}\right\rangle$, corresponding to diagrams $(m)-(q)$ in Fig. 9. The three lines in each diagram correspond to the cases listed in Eq. (2.30).

\subsubsection{Fermions and Spinless Adjoints}

Before studying the structure of localized KK-number violating terms associated with the spinless adjoints, we compute the effect of the interactions of the spinless adjoints with scalar and fermionic matter. In this subsection we give the result of the fermion loops, and in the next we consider scalar loops.

The contribution due to fermions to the two-point function $\left\langle A_{+} A_{+}^{\dagger}\right\rangle$ is given by (see Fig. 8)

$$
(a)=-\left(\frac{1}{4}\right) g_{4}^{2} i^{2} T(\Psi) \delta_{a b} \sum_{m_{1}, l_{1}} \sum_{m_{1}^{\prime}, l_{1}^{\prime}} \int \frac{d^{D} k}{(2 \pi)^{D}} \operatorname{Tr}\left\{\Gamma^{+} G_{k+p}^{ \pm,\left(m_{1}, l_{1} ; m_{1}^{\prime}, l_{1}^{\prime}\right)} \Gamma^{-} G_{k}^{ \pm,\left(m_{2}^{\prime}, l_{2}^{\prime} ; m_{2}, l_{2}\right)} P_{\mp}\right\},
$$

where $\operatorname{Tr}\left(T^{a} T^{b}\right)=T(\Psi) \delta_{a b}$. Assuming that the fermion has a zero-mode (of any $4 \mathrm{D}$ chirality), we obtain

$$
-i \frac{g_{4}^{2}}{16 \pi^{2}} T(\Psi) \delta_{a b} \Gamma\left(\frac{\epsilon}{2}\right) r_{m, l} r_{m^{\prime}, l^{\prime}}^{*} M_{m, l} M_{m^{\prime}, l^{\prime}} B_{+},
$$

with $B_{+}=-2$.

The fermion loop $(l)$ in Fig. 9 is given by Eq. (5.96) with $\Gamma^{+} \rightarrow \Gamma^{-}$. Assuming that the fermion has a zero-mode (of any $4 \mathrm{D}$ chirality), we obtain

$$
-i \frac{g_{4}^{2}}{16 \pi^{2}} T(\Psi) \delta_{a b} \Gamma\left(\frac{\epsilon}{2}\right) r_{m, l^{\prime}}^{*} r_{m^{\prime}, l^{\prime}}^{*} M_{m, l} M_{m^{\prime}, l^{\prime}} \tilde{B}_{+},
$$

with $\tilde{B}_{+}=2$. As for the diagrams arising from the gauge self-interactions, the fermion loop gives $\tilde{B}_{+}=-B_{+}$, which implies a gauge invariant counterterm.

\subsubsection{Scalars and Spinless Adjoints}

We finally consider the effect of scalar fields interacting with spinless adjoints via the vertices of Figure 7. As shown in Figure 10, there is a diagram involving a trilinear interaction,

$$
(u)=-\left(\frac{1}{4}\right)^{2} g_{4}^{2} T(\Phi) \delta_{a b} \sum_{m_{1}, l_{1}} \sum_{m_{1}^{\prime}, l_{1}^{\prime}} \int \frac{d^{D} k}{(2 \pi)^{D}}\left[r_{m_{1}, l_{1}} M_{m_{1}, l_{1}}+r_{m_{2}, l_{2}} M_{m_{2}, l_{2}}\right]
$$




\begin{tabular}{|c|c|c|c|c|c|c|c|c|}
\hline & \multicolumn{4}{|c|}{$B_{+}$} & \multicolumn{4}{|c|}{$B_{+}^{\prime}$} \\
\hline & & $n=0$ & $n=1$, & $n=2$ & & $n=0$ & $n=1,3$ & $n=2$ \\
\hline \multirow{4}{*}{ (u) } & \multirow{4}{*}{$-\frac{1}{4} \times$} & -2 & 0 & 2 & \multirow{4}{*}{$-\frac{1}{4} \times$} & 0 & 0 & 0 \\
\hline & & -2 & 0 & 2 & & 0 & 0 & 0 \\
\hline & & -2 & 0 & 2 & & $1 / 2$ & $-1 / 2$ & $1 / 2$ \\
\hline & & -2 & 0 & 2 & & $-1 / 2$ & $1 / 2$ & $-1 / 2$ \\
\hline \multirow{4}{*}{ (v) } & \multirow{4}{*}{$(-1) \times$} & $-5 / 4$ & $1 / 4$ & $3 / 4$ & \multirow{4}{*}{$(-1) \times$} & 0 & 0 & 0 \\
\hline & & -1 & 0 & 1 & & 0 & 0 & 0 \\
\hline & & $-9 / 8$ & $1 / 8$ & $7 / 8$ & & $-1 / 8$ & $1 / 8$ & $-1 / 8$ \\
\hline & & $-9 / 8$ & $1 / 8$ & $7 / 8$ & & $1 / 8$ & $-1 / 8$ & $1 / 8$ \\
\hline
\end{tabular}

Table 9: Functions $B_{+}$and $B_{+}^{\prime}$, as defined via Eq. (5.101), associated with the scalar contributions to the two-point function $\left\langle A_{+} A_{+}^{\dagger}\right\rangle$, corresponding to diagrams $(u)$ and $(v)$ in Fig. 10. We give the results for scalars satisfying any of the four types of boundary conditions, $n=0,1,2$ or 3 . The four lines in each diagram correspond to cases 1a, 1b, 2a and $2 \mathrm{~b}$ of Eqs. (2.30) and (2.32).

$$
\times G_{k+p, n}^{\left(m_{1}, l_{1} ; m_{1}^{\prime}, l_{1}^{\prime}\right)}\left[r_{m_{1}^{\prime}, l_{1}^{\prime}}^{*} M_{m_{1}^{\prime}, l_{1}^{\prime}}+r_{m_{2}^{\prime}, l_{2}^{\prime}}^{*} M_{m_{2}^{\prime}, l_{2}^{\prime}}\right] G_{k, n}^{\left(m_{2}^{\prime}, l_{2}^{\prime} ; m_{2}, l_{2}\right)},
$$

and one involving a quartic interaction:

$$
(v)=-i g_{4}^{2} T(\Phi) \delta_{a b} \sum_{m_{1}, l_{1}} \int \frac{d^{D} k}{(2 \pi)^{D}} G_{k, n}^{\left(m_{1}, l_{1} ; m_{1}^{\prime}, l_{1}^{\prime}\right)}
$$

where $\operatorname{Tr}\left(T^{a} T^{b}\right)=T(\Phi) \delta_{a b}$. We can write these diagrams as

$$
-i \frac{g_{4}^{2}}{16 \pi^{2}} T(\Phi) \delta_{a b} \Gamma\left(\frac{\epsilon}{2}\right) M_{m, l} M_{m^{\prime}, l^{\prime}}\left\{B_{+} r_{m, l} r_{m^{\prime}, l^{\prime}}^{*}+B_{+}^{\prime} r_{m, l^{\prime}}^{*} r_{m^{\prime}, l^{\prime}}\right\}
$$

with the scalar coefficients $B_{+}, B_{+}^{\prime}$ given in Table 9. Adding the diagrams, we get for scalars

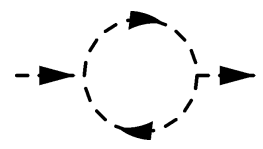

(u)

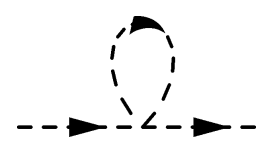

$(\mathrm{v})$

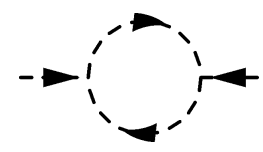

$(\mathrm{w})$

Figure 10: One-loop contribution to $\left\langle A_{+} A_{+}^{\dagger}\right\rangle$ and $\left\langle A_{+} A_{+}\right\rangle$due to scalars interacting with the spinless adjoints via the $6 \mathrm{D}$ gauge interactions of Figure $\mathrm{\theta}$. 
satisfying any of the four types of boundary conditions, $n=0,1,2$ or 3 :

$$
B_{+}=\left\{\begin{array}{ccc}
\mathrm{n}=0 & \mathrm{n}=1,3 & \mathrm{n}=2 \\
7 / 4 & -1 / 4 & -5 / 4 \\
3 / 2 & 0 & -3 / 2 \\
13 / 8 & -1 / 8 & -11 / 8
\end{array}, \quad B_{+}^{\prime}=0,\right.
$$

so that again the rotationally violating contribution proportional to $B_{+}^{\prime}$ vanishes.

The contribution to the two-point function $\left\langle A_{+} A_{+}\right\rangle$from diagram $(w)$ in Figure 10 is given by Eq. (5.99) with $r_{m_{1}, l_{1}} \rightarrow r_{m_{1}, l_{1}}^{*}$ and $r_{m_{2}, l_{2}} \rightarrow r_{m_{2}, l_{2}}^{*}$, and the result can be written as

$$
-i \frac{g_{4}^{2}}{16 \pi^{2}} T(\Phi) \delta_{a b} \Gamma\left(\frac{\epsilon}{2}\right) r_{m, l}^{*} r_{m^{\prime}, l^{\prime}}^{*} M_{m, l} M_{m^{\prime}, l^{\prime}} \tilde{B}_{+},
$$

with $\tilde{B}_{+}=-B_{+}$, and $B_{+}$given in Eq. (5.102).

\subsubsection{Localized operators}

We computed in the previous subsections the contributions to the spinless adjoint two-point functions due to the gauge self-interactions, as well as to the interactions with fermions and scalars arising from the $6 \mathrm{D}$ gauge interactions. We showed that one obtains the structure

$$
\begin{aligned}
\left\langle A_{+}^{\left(m^{\prime}, l^{\prime}\right)} A_{+}^{(m, l) \dagger}\right\rangle & \sim B_{+} r_{m, l} r_{m^{\prime}, l^{\prime}}^{*} M_{m, l} M_{m^{\prime}, l^{\prime}} \\
\left\langle A_{+}^{\left(m^{\prime}, l^{\prime}\right)} A_{+}^{(m, l)}\right\rangle & \sim \tilde{B}_{+} r_{m, l}^{*} r_{m^{\prime}, l^{\prime}}^{*} M_{m, l} M_{m^{\prime}, l^{\prime}}
\end{aligned}
$$

with $\tilde{B}_{+}=-B_{+}$. It follows from this and the hermitian conjugates of relations (5.104), that the logarithmic divergences require the gauge invariant localized counterterm

$$
\frac{1}{4} \delta_{c_{+}}(z) \times\left(-\frac{1}{2} \hat{r}_{+} L^{2} F_{45}^{a} F_{45}^{a}\right)
$$

where $\delta_{c}(z)$ was defined in Eq. (2.21), the factor of $1 / 4$ accounts for universal KK wavefunction enhancements, and

$$
\begin{aligned}
-\frac{1}{2} F_{45}^{2} & =\frac{1}{8} F_{+-}^{2} \\
& =\frac{1}{8}\left[\partial_{+} A_{-}-\partial_{-} A_{+}\right]^{2} .
\end{aligned}
$$

Recalling the KK decompositions $A_{+}^{j, k}(x, z) \sim-A_{+}^{j, k}(x) f_{3}^{j, k}(z), A_{-}^{j, k}(x, z) \sim A_{-}^{j, k}(x) f_{1}^{j, k}(z)$, and using Eqs. (2.26), one can see that the tree-level contribution to $\left\langle A_{+}^{\left(m^{\prime}, l^{\prime}\right)} A_{+}^{(m, l) \dagger}\right\rangle$ associated with the vertex $(5.105)$ is

$$
-\frac{i}{4} r_{m, l} r_{m^{\prime}, l^{\prime}}^{*} M_{m, l} M_{m^{\prime}, l^{\prime}} \times\left\{\begin{array}{c}
\left(2+c_{+}\right) \hat{r}_{+} \\
\left(2-c_{+}\right) \hat{r}_{+} \\
2 \hat{r}_{+}
\end{array}\right.
$$


where the three lines correspond to the cases listed in Eq. (2.30). From this we can read the oneloop contributions to $\hat{r}_{+}$from the results of the previous subsections. The gauge self-interactions, Eqs. (5.92), (5.93), (5.94) and (5.95), give a contribution

$$
\hat{r}_{+}=8 \times \frac{g_{4}^{2}}{16 \pi^{2}} C_{2}(A) \Gamma\left(\frac{\epsilon}{2}\right), \quad c_{+}=\frac{1}{4},
$$

the fermions [see Eqs. (5.97), and (5.98)] give

$$
\hat{r}_{+}=-4 \times \frac{g_{4}^{2}}{16 \pi^{2}} T(\Psi) \Gamma\left(\frac{\epsilon}{2}\right), \quad c_{+}=0,
$$

and the scalars [see Eqs. (5.101), (5.102) and (5.103)] give

$$
\hat{r}_{+}=\frac{13}{4} \times \frac{g_{4}^{2}}{16 \pi^{2}} T(\Phi) \Gamma\left(\frac{\epsilon}{2}\right), \quad c_{+}=\frac{2}{13} .
$$

The previous results for the localized operators assume that the fermion and scalar fields include a zero-mode in their KK towers. The coefficients of the localized operators induced by scalar fields satisfying other boundary conditions can be easily read from the results presented in subsection 5.4.3.

\section{Summary and Conclusions}

We considered the one-loop structure of general field theories in six dimensions, with two of the dimensions compactified on the "chiral square" of Ref. [10]. This compactification has a very simple geometric description: start from a square region in the plane and identify adjacent sides of the square. This compactification to four dimensions has the desirable property of leading to a chiral four-dimensional theory, and is therefore appropriate for phenomenological applications. In fact, as shown in [10], the construction is equivalent to a $T^{2} / Z_{4}$ orbifold. The geometric construction makes it clear that there are three singular points with a conical symmetry. Our explicit one-loop computation shows that there are logarithmic divergences that require the introduction of counterterms precisely at these three points. It also shows that the localized counterterms obey a rotational symmetry, as expected from the conical nature of the singularities.

We derived the propagators for spin-0, spin- $1 / 2$ and spin- 1 fields in momentum space and showed how to take into account the "boundary conditions" that define the propagation of these fields on the chiral square background. Putting the information about the compactification in the form of the propagators permits the use of vertices that conserve momentum in the extra dimensions, and therefore allows us to consider arbitrary interactions in a universal manner.

We also considered the 4D spin-0 fields that arise from the extra dimensional components of $6 \mathrm{D}$ gauge fields. Their interactions among themselves and with other fermion and scalar fields are constrained by the $6 \mathrm{D}$ gauge invariance. These states are characteristic of the present class of six-dimensional theories. We find that the self-interactions give a positive mass shift, while the gauge interactions with fermions give a negative mass shift. This is similar to their spin-1 
counterparts. However, the numerical coefficients are different. When applied to the standard model field content, one finds that the lightest KK particle is the spinless adjoint associated with the hypercharge interactions. Thus, these scenarios give rise to a scalar dark matter candidate, with Yukawa-like couplings determined by the gauge interactions.

Our results can be summarized succinctly by giving the coefficients of the various quadratic operators involving the given fields. In order to do so, we define the following shorthand notation for the various kinetic term operators. For the $6 \mathrm{D}$ gauge system we have two types of kinetic terms:

$$
\mathcal{O}_{A}=-\frac{1}{4} F_{\mu \nu}^{a} F^{\mu \nu a}, \quad \mathcal{O}_{45}=-\frac{1}{2} F_{45}^{a} F_{45}^{a} .
$$

For $6 \mathrm{D}$ Weyl fermions, $\Psi_{ \pm}$, with a left-handed zero-mode, the kinetic terms generated at the singularities have the form

$$
\mathcal{O}_{\Psi_{L}}=i \bar{\Psi}_{ \pm} \Gamma^{\mu} P_{L} \partial_{\mu} \Psi_{ \pm}, \quad \mathcal{O}_{\Psi_{L}}^{M}=i \bar{\Psi}_{ \pm} \Gamma^{ \pm} P_{R} \partial_{\mp} \Psi_{ \pm}+\text {h.c. }
$$

while for $6 \mathrm{D}$ Weyl fermions with a right-handed zero-mode they have an analogous structure with $P_{L} \leftrightarrow P_{R}$. For scalar fields there are four types of localized kinetic term operators as shown in Eq. (2.22). However, for scalars having a zero-mode, i.e. satisfying $n=0$ boundary conditions, only two types of kinetic terms are generated:

$$
\mathcal{O}_{\Phi}=\partial_{\mu} \Phi^{\dagger} \partial^{\mu} \Phi, \quad \mathcal{O}_{\Phi}^{M}=\Phi^{\dagger}\left(\partial_{+} \partial_{-} \Phi\right)+\text { h.c. },
$$

In this case, there are also induced mass terms, $\Phi^{\dagger} \Phi$.

Assuming that the zero-mode fermion is left-handed, we found that the quantum effects induce localized kinetic term operators at the points $(0,0)$ and $(L, L)$, which we write as

$$
\begin{aligned}
\frac{L^{2}}{4}\left[\delta\left(x^{4}\right) \delta\left(x^{5}\right)+\delta\left(L-x^{4}\right) \delta\left(L-x^{5}\right)\right] & \times\left\{\hat{r}_{1}^{A} \mathcal{O}_{A}+\hat{r}_{1}^{45} \mathcal{O}_{45}\right. \\
& \left.+\hat{r}_{1}^{\Psi} \mathcal{O}_{\Psi_{L}}+\hat{r}_{1}^{\Psi, M} \mathcal{O}_{\Psi_{L}}^{M}+\hat{r}_{1}^{\Phi} \mathcal{O}_{\Phi}+\hat{r}_{1}^{\Phi, M} \mathcal{O}_{\Phi}^{M}\right\}
\end{aligned}
$$

where, for convenience, we wrote an explicit factor of $1 / 4$ to account for the KK wavefunction enhancements arising from Eq. (2.2) evaluated at the singular points. The coefficients of the operators at these two conical singularities are found to be identical, as required by KK-Parity. If bare contributions at the cutoff scale $\Lambda$ can be neglected, RG evolution induces coefficients that can be read from Eqs. (5.11), (5.19), (5.38), (5.58), (5.67), (5.78), (5.85), (5.108), (5.109) and $(5.110)$ :

$$
\begin{aligned}
& \hat{r}_{1}^{A}=\frac{g_{4}^{2}}{16 \pi^{2}} \ln \frac{\Lambda^{2}}{\mu^{2}}\left[-\frac{14}{3} C_{2}(A)+\frac{2}{3} \sum_{\Psi} T(\Psi)+\frac{5}{12} \sum_{\Phi} T(\Phi)\right], \\
& \hat{r}_{1}^{45}=\frac{g_{4}^{2}}{16 \pi^{2}} \ln \frac{\Lambda^{2}}{\mu^{2}}\left[8 C_{2}(A)-4 \sum_{\Psi} T(\Psi)+\frac{13}{4} \sum_{\Phi} T(\Phi)\right],
\end{aligned}
$$




$$
\begin{aligned}
\hat{r}_{1}^{\Psi} & =\frac{1}{16 \pi^{2}} \ln \frac{\Lambda^{2}}{\mu^{2}}\left[-4 \sum_{\text {gauge }} g_{4}^{2} C_{2}(\Psi)+\frac{5}{8} \sum_{i} \lambda_{4, i}^{2}\right], \\
\hat{r}_{1}^{\Psi, M} & =\frac{1}{16 \pi^{2}} \ln \frac{\Lambda^{2}}{\mu^{2}}\left[\frac{5}{8} \sum_{i} \lambda_{4, i}^{2}\right], \\
\hat{r}_{1}^{\Phi} & =\frac{1}{16 \pi^{2}} \ln \frac{\Lambda^{2}}{\mu^{2}}\left[-\frac{15}{2} \sum_{\text {gauge }} g_{4}^{2} C_{2}(\Phi)+\sum_{i} \lambda_{4, i}^{2}\right], \\
\hat{r}_{1}^{\Phi, M} & =\frac{1}{16 \pi^{2}} \ln \frac{\Lambda^{2}}{\mu^{2}}\left[-\frac{45}{16} \sum_{\text {gauge }} g_{4}^{2} C_{2}(\Phi)+\sum_{i} \lambda_{4, i}^{2}\right],
\end{aligned}
$$

where $\mu$ is the renormalization scale, and $g_{4}$ and $\lambda_{4, i}$ are the 4-dimensional gauge and Yukawa couplings, respectively. In the equations for $\hat{r}_{1}^{A}$ and $\hat{r}_{1}^{45}$ the first sum runs over $6 \mathrm{D}$ Weyl fermions, while the second runs over $6 \mathrm{D}$ complex scalars satisfying $n=0$ boundary conditions. $C_{2}(F)$ is the Casimir eigenvalue in the representation of the fields $F=A_{\mu}, \Psi$ or $\Phi$, while $\operatorname{Tr}\left(T^{a} T^{b}\right)=T(F) \delta_{a b}$, where $T^{a}$ are the generators in the representation of the field $F$. The terms proportional to $C_{2}(A)$ include the contributions of the complete $6 \mathrm{D}$ gauge multiplet, i.e. both the $4 \mathrm{D}$ spin- 1 components, as well as the spinless adjoints. The sums in the expression for $\hat{r}_{1}^{\Psi}$ and $r_{1}^{\Psi, M}$ run over its gauge interactions, as well as the Yukawa interactions with complex scalars satisfying $n=0$ boundary conditions. We also derived relations for scalars satisfying more general boundary conditions in Section 5.3 .

In addition, one finds operators at a third singular point with coordinates $(0, L)$ :

$$
\frac{L^{2}}{4} \delta\left(x^{4}\right) \delta\left(L-x^{5}\right)\left(\hat{r}_{2}^{A} \mathcal{O}_{A}+\hat{r}_{2}^{45} \mathcal{O}_{45}+\hat{r}_{2}^{\Psi} \mathcal{O}_{\Psi_{L}}+\hat{r}_{2}^{\Psi, M} \mathcal{O}_{\Psi_{L}}^{M}+\hat{r}_{2}^{\Phi} \mathcal{O}_{\Phi}+\hat{r}_{2}^{\Phi, M} \mathcal{O}_{\Phi}^{M}\right)
$$

where the coefficients are in general independent from those in Eq. (6.4). The contribution due to physics below the cutoff scale $\Lambda$ was found to be

$$
\begin{aligned}
\hat{r}_{2}^{A} & =\frac{g_{4}^{2}}{16 \pi^{2}} \ln \frac{\Lambda^{2}}{\mu^{2}}\left[-2 C_{2}(A)+\frac{1}{6} \sum_{\Phi} T(\Phi)\right], \\
\hat{r}_{2}^{45} & =\frac{g_{4}^{2}}{16 \pi^{2}} \ln \frac{\Lambda^{2}}{\mu^{2}}\left[2 C_{2}(A)+\frac{1}{2} \sum_{\Phi} T(\Phi)\right], \\
\hat{r}_{2}^{\Psi} & =\frac{1}{16 \pi^{2}} \ln \frac{\Lambda^{2}}{\mu^{2}}\left[-2 \sum_{\text {gauge }} g_{4}^{2} C_{2}(\Psi)+\frac{1}{4} \sum_{i} \lambda_{4, i}^{2}\right], \\
\hat{r}_{2}^{\Psi, M} & =\frac{1}{16 \pi^{2}} \ln \frac{\Lambda^{2}}{\mu^{2}}\left[\frac{1}{4} \sum_{i} \lambda_{4, i}^{2}\right] . \\
\hat{r}_{2}^{\Phi} & =\frac{1}{16 \pi^{2}} \ln \frac{\Lambda^{2}}{\mu^{2}}\left[-3 \sum_{\text {gauge }} g_{4}^{2} C_{2}(\Phi)\right],
\end{aligned}
$$




$$
\hat{r}_{2}^{\Phi, M}=\frac{1}{16 \pi^{2}} \ln \frac{\Lambda^{2}}{\mu^{2}}\left[-\frac{5}{8} \sum_{\text {gauge }} g_{4}^{2} C_{2}(\Phi)\right] .
$$

It should be noted that the above coefficients were obtained in an $R_{\xi}$-gauge with $\xi=-3$ (the expressions for the various two-point functions in an arbitrary gauge are given in the main text). This is a convenient gauge since all induced operators have automatically a gauge invariant structure. To see the gauge invariance explicitly for other choices of the gauge parameter, a further field redefinition is required. Of course, physical quantities that may be calculated from the two point functions, such as the mass shifts, are $\xi$-independent. See the discussion in subsection 5.1.4.

Although in this paper we did not compute explicitly the renormalization of KK-number violating gauge interactions, the result of such a computation in $\xi=-3$ gauge should give rise to operators with the precise coefficients necessary to provide the gauge invariant completions of the kinetic operators in Eqs. (6.1)-(6.3), according to the standard prescription $\partial_{M} \rightarrow D_{M}=$ $\partial_{M}-i A_{M}$. Thus, the operators given in Eqs. (6.4), (6.5), (6.6) and (6.7) provide a very convenient summary of the one-loop results computed in this paper, allowing a straightforward determination of the induced mass-shifts, or of any KK-number violating gauge interactions of interest.

For example, the leading corrections to the gauge boson masses can be obtained from

$$
M_{A^{(j, k)}}=M_{j, k}\left(1-\frac{1}{2} \delta \mathcal{Z}_{A^{(j, k)}}\right),
$$

where $\delta \mathcal{Z}_{A^{(j, k)}}$ is the wavefunction renormalization of $A_{\mu}^{(j, k)}$ coming from the localized operator $\mathcal{O}_{A}$ in Eq. (6.1), and the tree-level mass of the $(j, k)$-th level is given by $M_{j, k}=\sqrt{j^{2}+k^{2}} / R$. The spinless adjoints, on the other hand, receive only a "mass" renormalization associated with $\mathcal{O}_{45}$ in Eq. (6.1), since the $4 \mathrm{D}$ kinetic term operators vanish at the singular points. In fact, $\mathcal{O}_{45}$, when expanded in KK modes, contains precisely the gauge invariant linear combination of $A_{4}$ and $A_{5}$ that is orthogonal to the eaten would-be Nambu-Goldstone modes. Thus, only this physical degree of freedom receives a mass shift from the localized operators, given by

$$
M_{A_{S A}^{(j, k)}}=M_{j, k}\left(1+\frac{1}{2} \delta \mathcal{Z}_{A_{S A}^{(j, k)}}\right) .
$$

For fermions, one finds to first order in perturbation theory,

$$
M_{\Psi^{(j, k)}}=M_{j, k}\left(1-\frac{1}{2} \delta \mathcal{Z}_{\Psi^{(j, k)}}+\frac{1}{2} \delta \mathcal{Z}_{\Psi^{(j, k)}}^{\prime}\right),
$$

where $\delta \mathcal{Z}_{\Psi^{(j, k)}}$ is the localized $4 \mathrm{D}$ kinetic term renormalization constant, and $\delta \mathcal{Z}_{\Psi^{(j, k)}}^{\prime}$ the renormalization of the kinetic terms with transverse derivatives, i.e. mass renormalization in a KK language. Notice that only one of the $4 \mathrm{D}$ chiralities receives a wavefunction renormalization due to localized operators. For scalars, one similarly has

$$
M_{\Phi^{(j, k)}}^{2}=4 m_{0}^{2}+M_{j, k}^{2}\left(1-\delta \mathcal{Z}_{\Phi^{(j, k)}}+\delta \mathcal{Z}_{\Phi^{(j, k)}}^{\prime}\right),
$$


where $m_{0}$ is the mass of the zero-mode, and the factor of four arises from the normalization of the heavy KK states relative to the zero-mode.

For KK-parity even states, the $\delta \mathcal{Z}$ 's are related to the coefficients of the localized kinetic term operators of Eqs. (6.4) and (6.6) by

$$
\begin{aligned}
\delta \mathcal{Z}_{A^{(j, k)}}=2 \hat{r}_{1}^{A}+\hat{r}_{2}^{A}, & \delta \mathcal{Z}_{A_{S A}^{(j, k)}}=2 \hat{r}_{1}^{45}+\hat{r}_{2}^{45}, \\
\delta \mathcal{Z}_{\Psi^{(j, k)}}=2 \hat{r}_{1}^{\Psi}+\hat{r}_{2}^{\Psi}, & \delta \mathcal{Z}_{\Phi^{(j, k)}}=2 \hat{r}_{1}^{\Phi}+\hat{r}_{2}^{\Phi}, \\
\delta \mathcal{Z}_{\Psi^{(j, k)}}^{\prime}=2 \operatorname{Re}\left(2 \hat{r}_{1}^{\Psi, M}+\hat{r}_{2}^{\Psi, M}\right), & \delta \mathcal{Z}_{\Phi^{(j, k)}}^{\prime}=2 \operatorname{Re}\left(2 \hat{r}_{1}^{\Phi, M}+\hat{r}_{2}^{\Phi, M}\right) .
\end{aligned}
$$

For KK-parity odd states, the $\delta \mathcal{Z}$ 's are as in Eqs. (6.12) except that the $r_{2}$ 's do not contribute, since the corresponding KK wavefunctions vanish at $\left(x^{4}, x^{5}\right)=(0, L)$. The explicit mass formulae for both KK-parity even and KK-parity odd states were given in Eqs. (1.9)-(1.16) of the Introduction, where we also included the results for scalars satisfying boundary conditions other than $n=0$.

As mentioned before, the localized operators summarized in Eqs. (6.4)-(6.7) contain much more information than the mass shifts. They also encode information about KK transitions, as well as new interactions with the massive gauge fields. As an important example of KK-number violating couplings, we consider those between zero-mode fermions, $\psi$, and massive KK-parity even gauge bosons, $A_{\mu}^{(j, k)}$. We write the effective $4 \mathrm{D}$ coupling as

$$
g_{4} C_{j, k}^{\Psi A} \bar{\psi} \gamma^{\mu} A_{\mu}^{(j, k)} \psi
$$

where the dimensionless parameters $C_{j, k}^{\Psi A}$ are determined, to lowest order in perturbation theory, by the coefficients defined in Eqs. (6.5) and (6.7), as

$$
C_{j, k}^{\Psi A}=-\frac{1}{2} \delta \overline{\mathcal{Z}}_{A^{(j, k)}}+\frac{1}{2} \delta \overline{\mathcal{Z}}_{\Psi^{(j, k)}}-\frac{1}{2} \delta \overline{\mathcal{Z}}_{\Psi^{(j, k)}}^{\prime},
$$

where now

$$
\begin{aligned}
\delta \overline{\mathcal{Z}}_{A^{(j, k)}} & =2 \hat{r}_{2}^{A}+(-1)^{j} \hat{r}_{1}^{A} \\
\delta \overline{\mathcal{Z}}_{\Psi^{(j, k)}} & =2 \hat{r}_{2}^{\Psi}+(-1)^{j} \hat{r}_{1}^{\Psi} \\
\delta \overline{\mathcal{Z}}_{\Psi^{(j, k)}}^{\prime} & =2 \operatorname{Re}\left[2 \hat{r}_{2}^{\Psi, M}+(-1)^{j} \hat{r}_{1}^{\Psi, M}\right]
\end{aligned}
$$

Notice that when $j$ is even, the KK-number violating couplings, $C_{j, k}^{\Psi A}$, are simply related to the mass shifts of the heavy states involved. However, when $j$ is odd, $C_{j, k}^{\Psi A}$ depends on a different linear combination of $\hat{r}_{1}$ and $\hat{r}_{2}$ than the one appearing in the mass shifts, e.g. Eq. (6.12). These couplings may play a crucial role in discriminating these scenarios from other kinds of new physics [14].

Acknowledgements: We would like to thank Gustavo Burdman, H. C. Cheng and Bogdan Dobrescu for interesting discussions. This work was supported by DOE under contract DEFG02-92ER-40699. 


\section{A. Kaluza-Klein Number versus Momentum Space Representations}

In this Appendix we derive in detail the general relation between the KK-number and momentum space representations of a generic two-point function. We derive results that are sufficiently general to cover the cases arising in the treatment of fermion and gauge fields. In particular, we allow for two-point functions connecting fields that satisfy different boundary conditions, labeled by integers $n_{1}$ and $n_{2}$.

The procedure is straightforward: starting from the propagator in configuration space, $G\left(p ; z, z^{\prime}\right)$, satisfying the appropriate boundary conditions, one can either project on the KK wavefunctions, $f^{(j, k)}(z)$, given in Eq. (2.2), or on the momentum space wavefunctions, $h^{(m, l)}(z)$, given in Eq. (2.3). However, one must exert some care since the two sets of functions form complete sets on different spacetime regions, and the quantum numbers $(j, k)$ and $(m, l)$ cover different ranges.

\section{A.1 Diagonal Propagators}

We start with propagators that preserve Kaluza-Klein number and postpone the analysis of Kaluza-Klein number violation to the next subsection. Using Eq. (2.12) we can derive, for any two integers $n_{1}, n_{2} \in\{0,1,2,3\}$ and arbitrary expansion coefficients $\tilde{g}_{j, k}$, the identity

$$
\begin{aligned}
& \frac{1}{4 L^{2}} \int_{-L}^{L} d^{2} z d^{2} z^{\prime}\left[h^{(m, l)}(z)\right]^{*}\left(\frac{1}{4 L^{2}} \sum_{j, k} \tilde{g}_{j, k} f_{n_{1}}^{(j, k)}(z)\left[f_{n_{2}}^{(j, k)}\left(z^{\prime}\right)\right]^{*}\right) h^{\left(m^{\prime}, l^{\prime}\right)}\left(z^{\prime}\right) \\
& \quad=\sum_{j, k} \frac{1}{2\left[1+\delta_{j, 0} \delta_{k, 0}\right]} \hat{\delta}\left(m, l ; j, k ; n_{1}\right) \tilde{g}_{j, k} \frac{1}{2\left[1+\delta_{m^{\prime}, 0} \delta_{l^{\prime}, 0}\right]} \hat{\delta}\left(j, k ; m^{\prime}, l^{\prime} ; n_{2}\right) \\
& \quad=\frac{1}{4}\left[\tilde{g}_{m, l}+e^{i\left(\theta_{1}-\theta_{2}\right)} \tilde{g}_{-l, m}+e^{2 i\left(\theta_{1}-\theta_{2}\right)} \tilde{g}_{-m,-l}+e^{3 i\left(\theta_{1}-\theta_{2}\right)} \tilde{g}_{l,-m}\right] \frac{\hat{\delta}\left(m, l ; m^{\prime}, l^{\prime} ; n_{2}\right)}{\left[1+\delta_{m, 0} \delta_{l, 0}\right]^{2}}
\end{aligned}
$$

where $\theta_{i}=n_{i} \pi / 2$. The significance of the "tilde" notation in $\tilde{g}_{j, k}$ will become clear in the following paragraphs.

In Eq. (A.1) it was necessary to assume that the sums over $j$ and $k$ run unrestricted over all integer values. To use this identity, the simplest way to proceed is to extend the restricted sums one naturally encounters when working in the KK-number representation [see comments after Eq. (2.3)] to the whole range of integers. This can be achieved by noting that the KK wavefunctions defined in Eq. (2.2) satisfy the relations

$$
f_{n}^{(-j, k)}(z)=e^{i \theta} f_{n}^{(k, j)}(z), \quad f_{n}^{(-j,-k)}(z)=e^{2 i \theta} f_{n}^{(j, k)}(z), f_{n}^{(j,-k)}(z)=e^{3 i \theta} f_{n}^{(k, j)}(z)
$$

Therefore, for arbitrary coefficients $g_{j, k}$, one can write

$$
\sum_{j, k}^{\prime} g_{j, k} f_{n_{1}}^{(j, k)}(z)\left[f_{n_{2}}^{(j, k)}\left(z^{\prime}\right)\right]^{*}=\frac{1}{4} \sum_{j, k} \tilde{g}_{j, k} f_{n_{1}}^{(j, k)}(z)\left[f_{n_{2}}^{j, k}\left(z^{\prime}\right)\right]^{*}
$$


where, following our convention, the ' superscript in the sum on the left-hand-side indicates that it runs over the restricted range $j>0, k \geq 0$ and $j=k=0$, while the sum on the right-handside stands for a double sum over all integers. The "tilded" quantities $\tilde{g}_{j, k}$ are defined in terms of $g_{j, k}$ as follows:

$$
\tilde{g}_{j, k}=\left\{\begin{array}{ll}
g_{j, k} & \text { for } j>0, k \geq 0 \\
e^{-i\left(\theta_{1}-\theta_{2}\right)} g_{k,-j} & \text { for } j \leq 0, k>0 \\
e^{-2 i\left(\theta_{1}-\theta_{2}\right)} g_{-j,-k} & \text { for } j<0, k \leq 0 \\
e^{-3 i\left(\theta_{1}-\theta_{2}\right)} g_{-k, j} & \text { for } j \geq 0, k<0
\end{array},\right.
$$

and $\tilde{g}_{0,0}=4 g_{0,0}$. Then, using Eqs. (A.1), A.3) and the definition (A.4) one obtains

$$
\begin{aligned}
\frac{1}{4 L^{2}} \int_{-L}^{L} d^{2} z d^{2} z^{\prime}\left[h^{(m, l)}(z)\right]^{*}\left(\frac{1}{L^{2}} \sum_{j, k}^{\prime} g_{j, k} f_{n_{1}}^{(j, k)}(z)\left[f_{n_{2}}^{(j, k)}\left(z^{\prime}\right)\right]^{*}\right) & h^{\left(m^{\prime}, l^{\prime}\right)}\left(z^{\prime}\right) \\
& =\tilde{g}_{m, l} \frac{\hat{\delta}\left(m, l ; m^{\prime}, l^{\prime} ; n_{2}\right)}{\left[1+\delta_{m, 0} \delta_{l, 0}\right]^{2}}
\end{aligned}
$$

It follows that for a propagator with the general representation in KK-number space,

$$
G_{n_{1}, n_{2}}\left(p ; z ; z^{\prime}\right)=\frac{1}{L^{2}} \sum_{j, k}^{\prime} g_{j, k} f_{n_{1}}^{(j, k)}(z)\left[f_{n_{2}}^{(j, k)}\left(z^{\prime}\right)\right]^{*},
$$

and using Eq. (A.5), as well as the completeness relation

$$
\frac{1}{4 L^{2}} \sum_{m, l} h^{(m, l)}(z)\left[h^{(m, l)}\left(z^{\prime}\right)\right]^{*}=\delta^{(2)}\left(z-z^{\prime}\right),
$$

we can write

$$
\begin{aligned}
G_{n_{1}, n_{2}}\left(p ; z ; z^{\prime}\right) & =\int_{-L}^{L} d^{2} y d^{2} y^{\prime} \delta^{(2)}(z-y) G_{n_{1}, n_{2}}\left(p ; y ; y^{\prime}\right) \delta^{(2)}\left(y^{\prime}-z^{\prime}\right) \\
& =\frac{1}{4 L^{2}} \sum_{m, l} \sum_{m^{\prime}, l^{\prime}} G_{p, n_{1}, n_{2}}^{\left(m, l ; l^{\prime}, l^{\prime}\right)} h^{(m, l)}(z)\left[h^{\left(m^{\prime}, l^{\prime}\right)}\left(z^{\prime}\right)\right]^{*}
\end{aligned}
$$

with $G_{p, n_{1}, n_{2}}^{\left(m, l m^{\prime}, l^{\prime}\right)}$, as defined in Eq. (2.11), explicitly given by

$$
G_{p, n_{1}, n_{2}}^{\left(m, l, m^{\prime}\right)}=\tilde{g}_{m, l} \frac{\hat{\delta}\left(m, l ; m^{\prime}, l^{\prime} ; n_{2}\right)}{\left[1+\delta_{m, 0} \delta_{l, 0}\right]^{2}}=\tilde{g}_{m^{\prime}, l^{\prime}} \frac{\hat{\delta}\left(m, l ; m^{\prime}, l^{\prime} ; n_{1}\right)}{\left[1+\delta_{m, 0} \delta_{l, 0}\right]^{2}} .
$$

To obtain the second equality we used the relations

$$
\tilde{g}_{l^{\prime},-m^{\prime}}=e^{i\left(\theta_{1}-\theta_{2}\right)} \tilde{g}_{m^{\prime}, l^{\prime}}, \quad \tilde{g}_{-m^{\prime},-l^{\prime}}=e^{2 i\left(\theta_{1}-\theta_{2}\right)} \tilde{g}_{m^{\prime}, l^{\prime}}, \quad \tilde{g}_{-l^{\prime}, m^{\prime}}=e^{3 i\left(\theta_{1}-\theta_{2}\right)} \tilde{g}_{m^{\prime}, l^{\prime}},
$$

which follow from the definitions (A.4).

Specializing Eq (A.9) to the scalar case with $n_{1}=n_{2}=n$, Eqs. (2.4) and (2.5), one obtains the scalar result of Eq. (2.17). Recall that $\tilde{g}_{0,0}=4 g_{0,0}$, as stated after Eq. (A.4). 


\section{A.2 Kaluza-Klein Mixing}

Now we consider two-point functions with an arbitrary KK-number violating structure, as in Eq. (2.18):

$$
\begin{aligned}
G\left(p ; z ; z^{\prime}\right) & =\frac{1}{L^{2}} \sum_{j, k}^{\prime} \sum_{j^{\prime}, k^{\prime}}^{\prime} g_{(j, k) ;\left(j^{\prime}, k^{\prime}\right)} f_{n_{1}}^{(j, k)}(z)\left[f_{n_{2}}^{\left(j^{\prime}, k^{\prime}\right)}\left(z^{\prime}\right)\right]^{*} \\
& =\frac{1}{4 L^{2}} \sum_{m, l} \sum_{m^{\prime}, l^{\prime}} G_{n_{1}, n_{2}}^{\left(m, l ; m^{\prime}, l^{\prime}\right)} h^{(m, l)}(z)\left[h^{\left(m^{\prime}, l^{\prime}\right)}\left(z^{\prime}\right)\right]^{*} .
\end{aligned}
$$

As was done in Eq. A.3), we may extend the definition of the coefficients $g_{(j, k) ;\left(j^{\prime}, k^{\prime}\right)}$ in such a way that the summations over KK number can be taken over an unrestricted range: ${ }^{7}$

$$
\sum_{j, k}^{\prime} \sum_{j^{\prime}, k^{\prime}}^{\prime} g_{(j, k) ;\left(j^{\prime}, k^{\prime}\right)} f_{n_{1}}^{(j, k)}(z)\left[f_{n_{2}}^{\left(j^{\prime}, k^{\prime}\right)}\left(z^{\prime}\right)\right]^{*}=\frac{1}{16} \sum_{j, k} \sum_{j^{\prime}, k^{\prime}} \tilde{g}_{(j, k) ;\left(j^{\prime}, k^{\prime}\right)} f_{n_{1}}^{(j, k)}(z)\left[f_{n_{2}}^{\left(j^{\prime}, k^{\prime}\right)}\left(z^{\prime}\right)\right]^{*}
$$

In order to write in a compact form the required extension $\tilde{g}_{(j, k) ;\left(j^{\prime}, k^{\prime}\right)}$, we define a "reordering" function

$$
R(j, k)= \begin{cases}(j, k) & \text { if }(j, k) \in S_{0}=\{j>0, k \geq 0\} \\ (k,-j) & \text { if }(j, k) \in S_{1}=\{j \leq 0, k>0\} \\ (-j,-k) & \text { if }(j, k) \in S_{2}=\{j<0, k \leq 0\} \\ (-k, j) & \text { if }(j, k) \in S_{3}=\{j \geq 0, k<0\}\end{cases}
$$

and also $P(j, k)=\omega$ if $(j, k) \in S_{\omega}$, giving the quadrant to which $(j, k)$ belongs. In terms of these auxiliary functions the "tilde" operation is given by

$$
\begin{aligned}
\tilde{g}_{(j, k) ;\left(j^{\prime}, k^{\prime}\right)} & =g_{R(j, k) ; R\left(j^{\prime} k^{\prime}\right)} e^{-i P(j, k) \theta_{1}+i P\left(j^{\prime}, k^{\prime}\right) \theta_{2}}, & & \text { if }(j, k) \&\left(j^{\prime}, k^{\prime}\right) \neq(0,0) \\
\tilde{g}_{(j, k) ;(0,0)} & =4 g_{R(j, k) ;(0,0)} e^{-i P(j, k) \theta_{1}}, & & \text { if }(j, k) \neq(0,0) \\
\tilde{g}_{(0,0) ;\left(j^{\prime}, k^{\prime}\right)} & =4 g_{(0,0) ; R\left(j^{\prime} k^{\prime}\right)} e^{i P\left(j^{\prime}, k^{\prime}\right) \theta_{2}}, & & \text { if }\left(j^{\prime}, k^{\prime}\right) \neq(0,0) \\
\tilde{g}_{(0,0) ;(0,0)} & =16 g_{(0,0) ;(0,0)}, & &
\end{aligned}
$$

where $\theta_{i}=n_{i} \pi / 2$.

To relate the expansion coefficients $g_{(j, k) ;\left(j^{\prime}, k^{\prime}\right)}$ and $G_{n_{1}, n_{2}}^{\left(m, l ; m^{\prime}, l^{\prime}\right)}$ (KK-number and momentum bases, respectively) in the KK-number violating case, we can project Eq. (A.11) on momentum space, as in Eq. (2.11). With the help of Eqs. 2.12) and (2.14), and following a procedure similar to the one used to derive Eqs. (A.1) and (A.5), we find

$$
\begin{aligned}
G_{n_{1}, n_{2}}^{\left(m, l ; m^{\prime}, l^{\prime}\right)} & =\frac{1}{16} \sum_{j, k} \sum_{j^{\prime}, k^{\prime}} \frac{1}{\left[1+\delta_{j, 0} \delta_{k, 0}\right]} \hat{\delta}\left(m, l ; j, k ; n_{1}\right) \tilde{g}_{(j, k) ;\left(j^{\prime}, k^{\prime}\right)} \frac{1}{\left[1+\delta_{m^{\prime}, 0} \delta_{l^{\prime}, 0}\right]} \hat{\delta}\left(j^{\prime}, k^{\prime} ; m^{\prime}, l^{\prime} ; n_{2}\right) \\
& =\frac{1}{\left[1+\delta_{m, 0} \delta_{l, 0}\right]\left[1+\delta_{m^{\prime}, 0} \delta_{l^{\prime}, 0}\right]} \tilde{g}_{(m, l) ;\left(m^{\prime}, l^{\prime}\right)} .
\end{aligned}
$$

\footnotetext{
${ }^{7}$ Although $\tilde{g}_{(j, k) ;\left(j^{\prime}, k^{\prime}\right)}$ depends on $n_{1}$ and $n_{2}$, we do not indicate this dependence to avoid further notational cluttering.
} 
Note that Eq. (A.9) is a subcase of the previous relation.

Using the fact that $g_{(j, k) ;\left(j^{\prime}, k^{\prime}\right)}$ and $\tilde{g}_{(j, k) ;\left(j^{\prime}, k^{\prime}\right)}$ coincide when $j>0, k \geq 0$, one immediately obtains Eq. (2.19). Eqs. (2.20), involving zero-modes, are also immediately derived from Eq. (A.15) and the definitions (A.14) and (A.13).

\section{A.3 Useful Identities for the Generalized Functions $\hat{\delta}\left(m, l ; m^{\prime}, l^{\prime} ; n\right)$}

Here we record some useful relations involving the $\hat{\delta}$-function introduced in Eq. (2.14):

$$
\begin{aligned}
\hat{\delta}\left(m_{1}, l_{1} ; m_{2}, l_{2} ; n\right) & =\hat{\delta}\left(m_{2}, l_{2} ; m_{1}, l_{1} ;-n\right), \\
\hat{\delta}\left(-m_{1},-l_{1} ; m_{2}, l_{2} ; n\right) & =\hat{\delta}\left(m_{1}, l_{1} ;-m_{2},-l_{2} ; n\right) \\
& =\hat{\delta}\left(m_{1}, l_{1} ; m_{2}, l_{2} ;-n\right), \\
\hat{\delta}\left(m_{1}, l_{1} ; l_{2},-m_{2} ; n\right) & =e^{-i n \pi / 2} \hat{\delta}\left(m_{1}, l_{1} ; m_{2}, l_{2} ; n\right), \\
\hat{\delta}\left(m_{1}, l_{1} ;-m_{2},-l_{2} ; n\right) & =e^{-i n \pi} \hat{\delta}\left(m_{1}, l_{1} ; m_{2}, l_{2} ; n\right), \\
\hat{\delta}\left(m_{1}, l_{1} ;-l_{2}, m_{2} ; n\right) & =e^{-3 i n \pi / 2} \hat{\delta}\left(m_{1}, l_{1} ; m_{2}, l_{2} ; n\right) .
\end{aligned}
$$

We also note that the products of generalized $\hat{\delta}$ 's that appear in the diagrams (see Figure 1) can be simplified using

$$
\begin{aligned}
\sum_{m_{1}^{\prime}, l_{1}^{\prime}} \hat{\delta}\left(m_{1}, l_{1} ; m_{1}^{\prime}, l_{1}^{\prime} ; n_{1}\right) \hat{\delta}\left(m_{2}^{\prime}, l_{2}^{\prime} ; m_{2}, l_{2} ; n_{2}\right)=\hat{\delta}\left(m, l ; m^{\prime}, l^{\prime} ; n_{1}-n_{2}\right) \\
+e^{i \theta_{1}} \hat{\delta}\left(m-m_{1}-l_{1}, l-l_{1}+m_{1} ; m^{\prime}, l^{\prime} ; n_{1}-n_{2}\right) \\
+e^{2 i \theta_{1}} \hat{\delta}\left(m-2 m_{1}, l-2 l_{1} ; m^{\prime}, l^{\prime} ; n_{1}-n_{2}\right) \\
+e^{3 i \theta_{1}} \hat{\delta_{1}}\left(m-m_{1}+l_{1}, l-l_{1}-m_{1} ; m^{\prime}, l^{\prime} ; n_{1}-n_{2}\right)
\end{aligned}
$$

where $\theta_{1}=n_{1} \pi / 2$, and $m_{2}=m_{1}-m, l_{2}=l_{1}-l, m_{2}^{\prime}=m_{1}^{\prime}-m^{\prime}$ and $l_{2}^{\prime}=l_{1}^{\prime}-l^{\prime}$.

One can check that the last three terms in Eq. (A.17), when summed over $\left(m_{1}, l_{1}\right)$ vanish, except when KK-parity is preserved and

- $n_{1}=n_{2}=0$ :

$$
\sum_{m_{1}, l_{1}}\left(\sum_{m_{1}^{\prime}, l_{1}^{\prime}} \hat{\delta}\left(m_{1}, l_{1} ; m_{1}^{\prime}, l_{1}^{\prime} ; 0\right) \hat{\delta}\left(m_{2}^{\prime}, l_{2}^{\prime} ; m_{2}, l_{2} ; 0\right)-\hat{\delta}\left(m, l ; m^{\prime}, l^{\prime} ; 0\right)\right)=4 \times\left\{\begin{array}{l}
3 \\
2 \\
5 / 2
\end{array}\right.
$$

- $n_{1}=n_{2}= \pm 1$ :

$$
\sum_{m_{1}, l_{1}}\left(\sum_{m_{1}^{\prime}, l_{1}^{\prime}} \hat{\delta}\left(m_{1}, l_{1} ; m_{1}^{\prime}, l_{1}^{\prime} ; \pm 1\right) \hat{\delta}\left(m_{2}^{\prime}, l_{2}^{\prime} ; m_{2}, l_{2} ; \pm 1\right)-\hat{\delta}\left(m, l ; m^{\prime}, l^{\prime} ; 0\right)\right)=4 \times\left\{\begin{array}{l}
-1 \\
0 \\
-1 / 2
\end{array}\right.
$$

- $n_{1}=n_{2}=2$ :

$$
\sum_{m_{1}, l_{1}}\left(\sum_{m_{1}^{\prime}, l_{1}^{\prime}} \hat{\delta}\left(m_{1}, l_{1} ; m_{1}^{\prime}, l_{1}^{\prime} ; 2\right) \hat{\delta}\left(m_{2}^{\prime}, l_{2}^{\prime} ; m_{2}, l_{2} ; 2\right)-\hat{\delta}\left(m, l ; m^{\prime}, l^{\prime} ; 0\right)\right)=4 \times\left\{\begin{array}{l}
-1 \\
-2 \\
-3 / 2
\end{array},\right.
$$


where the three cases in each of the bullets are as defined in Eq. (2.30), which we quote here again for convenience:

Case 1a: $\quad(-1)^{m+l}=(-1)^{m^{\prime}+l^{\prime}}=+1, \quad m-m^{\prime}$ even,

Case 1b: $\quad(-1)^{m+l}=(-1)^{m^{\prime}+l^{\prime}}=+1, \quad m-m^{\prime}$ odd,

Case 2: $\quad(-1)^{m+l}=(-1)^{m^{\prime}+l^{\prime}}=-1$,

or

- $n_{1}=0, n_{2}=2$ or $n_{1}=2, n_{2}=0$ :

$$
\sum_{m_{1}, l_{1}}\left(\sum_{m_{1}^{\prime}, l_{1}^{\prime}} \hat{\delta}\left(m_{1}, l_{1} ; m_{1}^{\prime}, l_{1}^{\prime} ; 0\right) \hat{\delta}\left(m_{2}^{\prime}, l_{2}^{\prime} ; m_{2}, l_{2} ; 2\right)-\hat{\delta}\left(m, l ; m^{\prime}, l^{\prime} ; 2\right)\right)=4 \times\left\{\begin{array}{l}
0 \\
1 / 2 \\
-1 / 2
\end{array},\right.
$$

where in this latter bullet the three cases are different from the previous ones, as given in Eq. (2.32):

Case 1: $\quad(-1)^{m+l}=(-1)^{m^{\prime}+l^{\prime}}=+1$,

Case 2a: $\quad(-1)^{m+l}=(-1)^{m^{\prime}+l^{\prime}}=-1, \quad m-m^{\prime}$ even,

Case 2b: $\quad(-1)^{m+l}=(-1)^{m^{\prime}+l^{\prime}}=-1, \quad m-m^{\prime}$ odd.

\section{B. Tree-level Propagators on the Chiral Square}

In this Appendix we derive the propagators for fields of various spins on the "chiral square" background of [10, 11. We follow the general strategy of deriving the propagator in the mixed position and momentum space representation, making use of the KK wavefunctions in Eq. (2.2) to take care of the appropriate boundary conditions, after which it is a simple matter to find the corresponding momentum space expressions using the formulae derived in Appendix $\mathrm{A}$.

\section{B.1 Chiral Fermions}

We start by computing the fermion propagator in mixed position and momentum space. We need to solve

$$
i\left(-i \Gamma^{\mu} p_{\mu}+\Gamma^{4} \partial_{4}+\Gamma^{5} \partial_{5}\right) G^{ \pm}\left(p ; z ; z^{\prime}\right)=i P_{\mp} \delta^{(2)}\left(z-z^{\prime}\right)
$$

where the \pm superscript in $G^{ \pm}\left(p ; z ; z^{\prime}\right)$ refers to the two possible $6 \mathrm{D}$ chiralities defined by

$$
P_{ \pm}=\frac{1}{2}(1 \pm \bar{\Gamma})
$$

and the six-dimensional chirality operator is $\bar{\Gamma}=\Gamma^{0} \Gamma^{1} \Gamma^{2} \Gamma^{3} \Gamma^{4} \Gamma^{5}$. A convenient representation of the $8 \times 8 \Gamma$-matrices is

$$
\Gamma^{\mu}=\gamma^{\mu} \otimes \sigma^{0}, \quad \Gamma^{4,5}=i \gamma_{5} \otimes \sigma^{1,2}
$$


where $\gamma^{\mu}$ are the $4 \mathrm{D} \gamma$-matrices, $\gamma_{5}=i \gamma^{0} \gamma^{1} \gamma^{2} \gamma^{3}$ is the $4 \mathrm{D}$ chirality operator, $\sigma^{0}$ is the $2 \times 2$ unit matrix and $\sigma^{i}$ are the Pauli matrices. In this representation, $\bar{\Gamma}=-\gamma_{5} \otimes \sigma^{3}$.

Each 6D chiral fermion contains both left- and right-handed 4-dimensional chiralities. Since the folded square identifications require them to obey different boundary conditions, it is useful to treat them separately by using the $8 \times 84 \mathrm{D}$ chirality projectors

$$
P_{L, R}=\frac{1}{2}\left(1 \mp i \Gamma^{0} \Gamma^{1} \Gamma^{2} \Gamma^{3}\right) \text {. }
$$

Defining

$$
\begin{aligned}
& G_{L L}^{ \pm}\left(p ; z, z^{\prime}\right)=P_{L} G^{ \pm}\left(p ; z, z^{\prime}\right) P_{R}=\int d^{4} x e^{i p x}\left\langle\Psi_{ \pm_{L}}(x, z) \overline{\Psi_{ \pm_{L}}}\left(0, z^{\prime}\right)\right\rangle \\
& G_{R L}^{ \pm}\left(p ; z, z^{\prime}\right)=P_{R} G^{ \pm}\left(p ; z, z^{\prime}\right) P_{R}=\int d^{4} x e^{i p x}\left\langle\Psi_{ \pm_{R}}(x, z) \overline{\Psi_{ \pm_{L}}}\left(0, z^{\prime}\right)\right\rangle \\
& G_{L R}^{ \pm}\left(p ; z, z^{\prime}\right)=P_{L} G^{ \pm}\left(p ; z, z^{\prime}\right) P_{L}=\int d^{4} x e^{i p x}\left\langle\Psi_{ \pm_{L}}(x, z) \overline{\Psi_{ \pm_{R}}}\left(0, z^{\prime}\right)\right\rangle \\
& G_{R R}^{ \pm}\left(p ; z, z^{\prime}\right)=P_{R} G^{ \pm}\left(p ; z, z^{\prime}\right) P_{L}=\int d^{4} x e^{i p x}\left\langle\Psi_{ \pm_{R}}(x, z) \overline{\Psi_{ \pm_{R}}}\left(0, z^{\prime}\right)\right\rangle
\end{aligned}
$$

we can derive the equations obeyed by $G_{L L}^{ \pm}, G_{R L}^{ \pm}$, etc. as follows. Applying the differential operator $i\left(-i \Gamma^{\mu} p_{\mu}+\Gamma^{4} \partial_{4}+\Gamma^{5} \partial_{5}\right)$ to Eq. (B.1), and then projecting by $P_{L}$ on the left and by $P_{R}$ on the right, we can obtain a differential equation for $G_{L L}^{ \pm}$:

$$
\left(p^{2}+\partial_{4}^{2}+\partial_{5}^{2}\right) G_{L L}^{ \pm}\left(p ; z, z^{\prime}\right)=i P_{L} P_{ \pm} \Gamma^{\mu} p_{\mu} \delta^{(2)}\left(z-z^{\prime}\right)
$$

where we used the fact that $\Gamma^{4}$ and $\Gamma^{5}$ commute with $P_{L, R}$. We see that this equation is solved by

$$
G_{L L}^{ \pm}\left(p ; z, z^{\prime}\right)=P_{L} P_{ \pm} \Gamma^{\mu} p_{\mu} G_{n_{L}^{ \pm}}\left(p ; z, z^{\prime}\right)
$$

where $G_{n_{L}^{ \pm}}\left(p ; z, z^{\prime}\right)$ is the 6-dimensional scalar propagator satisfying Eq. (2.7). It is given explicitly by

$$
G_{n_{L}^{ \pm}}\left(p ; z, z^{\prime}\right)=\frac{1}{L^{2}} \sum_{j, k}^{\prime} g_{S}^{j, k} f_{n_{L}^{ \pm}}^{(j, k)}(z)\left[f_{n_{L}^{ \pm}}^{(j, k)}\left(z^{\prime}\right)\right]^{*}
$$

where $g_{S}^{j, k}$ is the 4-dimensional scalar propagator defined in Eq. (2.5). The integers $n_{L}^{ \pm}$label the boundary conditions obeyed by the left-handed components of the $6 \mathrm{D}$ fermion in question.

We can find $G_{R L}^{ \pm}$from the solution $G_{L L}^{ \pm}$above by projecting directly Eq. (B.1), $P_{L} \cdots P_{R}$, to obtain

$$
\Gamma^{\mu} p_{\mu} G_{R L}^{ \pm}+i\left(\Gamma^{4} \partial_{4}+\Gamma^{5} \partial_{5}\right) G_{L L}^{ \pm}=0
$$

Using the identities Eqs. (5.16), we find

$$
\begin{aligned}
G_{R L}^{ \pm} & =-\frac{i}{p^{2}} p_{\mu} \Gamma^{\mu} \Gamma^{4} \partial_{ \pm} G_{L L}^{ \pm} \\
& =P_{R} P_{ \pm} \Gamma^{4}\left(i \partial_{ \pm} G_{n_{L}^{ \pm}}\right)
\end{aligned}
$$


where $\partial_{ \pm}$were defined in Eq. (2.23), and $G_{n_{L}^{ \pm}}$is given in Eq. (B.8).

Proceeding in an analogous fashion (i.e. projecting by $P_{R}$ on the left and by $P_{L}$ on the right) we see that $G_{R R}^{ \pm}$is given by

$$
G_{R R}^{ \pm}\left(p ; z, z^{\prime}\right)=P_{R} P_{ \pm} \Gamma^{\mu} p_{\mu} G_{n_{R}^{ \pm}}\left(p ; z, z^{\prime}\right)
$$

where $G_{n_{R}^{ \pm}}\left(p ; z, z^{\prime}\right)$ is given by Eq. ( $\left.\mathrm{B.8}\right)$ with $n_{L}^{ \pm} \rightarrow n_{R}^{ \pm}$, and also that $G_{L R}^{ \pm}$is given by

$$
G_{L R}^{ \pm}=P_{L} P_{ \pm} \Gamma^{4}\left(i \partial_{\mp} G_{n_{R}^{ \pm}}\right)
$$

The integers $n_{L}^{ \pm}$and $n_{R}^{ \pm}$associated with a given $6 \mathrm{D}$ fermion are related by Eq. (3.2). Using Eqs. (2.26) and (B.8) we can easily calculate the partial derivatives needed in Eqs. (B.10) and (B.12):

$$
i \partial_{ \pm} G_{n}\left(p ; z, z^{\prime}\right)=-\frac{1}{L^{2}} \sum_{j, k}^{\prime} r_{j, \pm k} M_{j, k} g_{S}^{j, k} f_{n \mp 1}^{(j, k)}(z)\left[f_{n}^{(j, k)}\left(z^{\prime}\right)\right]^{*}
$$

where the phases $r_{j, k}$ were defined in Eq. (2.27).

Putting the results $(\overline{B .7}),(\overline{B .10}),(\overline{B .11})$ and $(\overline{B .12})$ together, we see that the fermion propagator has the representation

$$
\begin{aligned}
& G_{L L}^{ \pm}\left(p ; z, z^{\prime}\right)=P_{L} P_{ \pm} \frac{1}{L^{2}} \sum_{j, k}^{\prime} \Gamma^{\mu} p_{\mu} g_{S}^{j, k}\left[f_{n_{L}^{ \pm}}^{(j, k)}(z)\right]\left[f_{n_{L}^{ \pm}}^{(j, k)}\left(z^{\prime}\right)\right]^{*} \\
& G_{R L}^{ \pm}\left(p ; z, z^{\prime}\right)=-P_{R} P_{ \pm} \frac{1}{L^{2}} \sum_{j, k}^{\prime} \Gamma^{4} M_{j, k} g_{S}^{j, k}\left[r_{j, \pm k} f_{n_{R}^{ \pm}}^{(j, k)}(z)\right]\left[f_{n_{L}^{ \pm}}^{(j, k)}\left(z^{\prime}\right)\right]^{*} \\
& G_{L R}^{ \pm}\left(p ; z, z^{\prime}\right)=-P_{L} P_{ \pm} \frac{1}{L^{2}} \sum_{j, k}^{\prime} \Gamma^{4} M_{j, k} g_{S}^{j, k}\left[f_{n_{L}^{ \pm}}^{(j, k)}(z)\right]\left[r_{j, \pm k} f_{n_{R}^{ \pm}}^{(j, k)}\left(z^{\prime}\right)\right]^{*} \\
& G_{R R}^{ \pm}\left(p ; z, z^{\prime}\right)=P_{R} P_{ \pm} \frac{1}{L^{2}} \sum_{j, k}^{\prime} \Gamma^{\mu} p_{\mu} g_{S}^{j, k}\left[r_{j, \pm k} f_{n_{R}^{ \pm}}^{(j, k)}(z)\right]\left[r_{j, \pm k} f_{n_{R}^{ \pm}}^{(j, k)}\left(z^{\prime}\right)\right]^{*}
\end{aligned}
$$

where $n_{L}^{ \pm}$and $n_{R}^{ \pm}$are related as in Eq. (3.2).

We may now project Eqs. (B.14) on momentum space, as in Eq. (2.11). Using Eqs. (A.4) and (A.5), we obtain

$$
\begin{aligned}
& G_{L L, p}^{ \pm,\left(m, l ; m^{\prime}, l^{\prime}\right)}=P_{L} P_{ \pm} \Gamma^{\mu} p_{\mu} g_{S}^{m, l} \hat{\delta}\left(m, l ; m^{\prime}, l^{\prime} ; n_{L}^{ \pm}\right) \\
& G_{R L, p}^{ \pm,\left(m, l ; m^{\prime}, l^{\prime}\right)}=-P_{R} P_{ \pm} \Gamma^{4} r_{m, \pm l} M_{m, l} g_{S}^{m, l} \hat{\delta}\left(m, l ; m^{\prime}, l^{\prime} ; n_{L}^{ \pm}\right) \\
& G_{L R, p}^{ \pm,\left(m, l ; m^{\prime}, l^{\prime}\right)}=-P_{L} P_{ \pm} \Gamma^{4} r_{m, \mp l} M_{m, l} g_{S}^{m, l} \hat{\delta}\left(m, l ; m^{\prime}, l^{\prime} ; n_{R}^{ \pm}\right) \\
& G_{R R, p}^{ \pm,\left(m, l ; m^{\prime}, l^{\prime}\right)}=P_{R} P_{ \pm} \Gamma^{\mu} p_{\mu} g_{S}^{m, l} \hat{\delta}\left(m, l ; m^{\prime}, l^{\prime} ; n_{R}^{ \pm}\right) .
\end{aligned}
$$

Note that by using the relations (3.2) the "tilde" operation defined in Eq. (A.4) simplifies considerably, and there is no need to distinguish among the four possible sign assignments of the momenta $m, l$. 
By adding the four results in Eq. (B.15), and using again the identities (5.16), we can write the fermion propagator in the more compact form given in Eq. (3.3). Recall that the extra-dimensional momenta with lower indices are given by $p_{4}=-m / R$ and $p_{5}=-l / R$.

\section{B.2 Gauge Fields: The Spin-1 Components}

After integration by parts, the terms involving $A^{\mu}$ in Eq. (4.1), with the gauge fixing Eq. (4.2), can be written as

$$
-\frac{1}{2} A^{\mu}\left[\left(p^{2}+\partial_{4}^{2}+\partial_{5}^{2}\right) \eta_{\mu \nu}-\left(1-\frac{1}{\xi}\right) p_{\mu} p_{\nu}\right] A^{\nu}
$$

where we went to the momentum space associated with the non-compact dimensions.

The spin-1 propagator in the mixed representation is defined by

$$
\left[\left(p^{2}+\partial_{4}^{2}+\partial_{5}^{2}\right) \eta_{\mu \lambda}-\left(1-\frac{1}{\xi}\right) p_{\mu} p_{\lambda}\right] G^{\lambda \nu}\left(p ; z ; z^{\prime}\right)=-i \delta_{\mu}^{\nu} \delta^{(2)}\left(z-z^{\prime}\right)
$$

and the solution satisfying the boundary conditions is

$$
G_{\mu \nu}\left(p ; z ; z^{\prime}\right)=\frac{1}{L^{2}} \sum_{j, k}^{\prime} g_{\mu \nu}^{j, k} f_{0}^{(j, k)}(z)\left[f_{0}^{(j, k)}\left(z^{\prime}\right)\right]^{*}
$$

with $g_{\mu \nu}^{j, k}$ as given in Eq. 4.5).

Going to momentum space in the compactified dimensions, as in Eq. (2.11), and using Eq. (A.9) with $n_{1}=n_{2}=0$, we can immediately derive Eq. (4.4).

\section{B.3 Gauge Fields: The Spin-0 Components}

In this subsection, we concentrate on the slightly trickier issues associated with the scalar degrees of freedom contained in the six-dimensional gauge field, $A_{M}$. Up to surface terms that do not contribute as a result of the boundary conditions discussed in Ref. [11, the terms in Eq. (4.1) [with the gauge fixing Eq. (4.2)] quadratic in $A_{4}, A_{5}$ can be written as

$$
\begin{aligned}
\mathcal{L} & \supset \frac{1}{2} \sum_{i, j=4,5} A^{i}\left[\left(p^{2}+\partial_{4}^{2}+\partial_{5}^{2}\right) \delta_{i j}-(1-\xi) \partial_{i} \partial_{j}\right] A^{j} \\
& =\frac{1}{4}\left(A_{+}^{*}, A_{-}^{*}\right)\left(\begin{array}{cc}
p^{2}+\frac{1}{2}(1+\xi) \partial_{+} \partial_{-} & -\frac{1}{2}(1-\xi) \partial_{+}^{2} \\
-\frac{1}{2}(1-\xi) \partial_{-}^{2} & p^{2}+\frac{1}{2}(1+\xi) \partial_{+} \partial_{-}
\end{array}\right)\left(\begin{array}{c}
A_{+} \\
A_{-}
\end{array}\right),
\end{aligned}
$$

where $\partial_{ \pm}$were defined in Eq. (2.23) and we wrote the second line in the $A_{ \pm}$basis as defined in Eq. (4.3). The fields $A_{ \pm}$are convenient since they satisfy well defined boundary conditions [11]:

$$
A_{ \pm}\left(x^{\mu} ; z\right)=\mp \frac{1}{L} \sum_{j, k}^{\prime} A_{ \pm}^{(j, k)}\left(x^{\mu}\right) f_{3,1}^{(j, k)}(z)
$$

We define the propagator for the $A_{ \pm}$system by

$$
\frac{1}{2}\left(\begin{array}{cc}
p^{2}+\frac{1}{2}(1+\xi) \partial_{+} \partial_{-} & -\frac{1}{2}(1-\xi) \partial_{+}^{2} \\
-\frac{1}{2}(1-\xi) \partial_{-}^{2} & p^{2}+\frac{1}{2}(1+\xi) \partial_{+} \partial_{-}
\end{array}\right)\left(\begin{array}{c}
G_{++} G_{+-} \\
G_{-+} G_{--}
\end{array}\right)=i \delta^{(2)}\left(z-z^{\prime}\right)
$$


To find the solution to Eq. (B.21) we make the following ansatz

$$
\begin{aligned}
& G_{++}\left(p ; z ; z^{\prime}\right)=\frac{1}{L^{2}} \sum_{j, k}^{\prime} g_{++}^{j, k} f_{3}^{(j, k)}(z)\left[f_{3}^{(j, k)}\left(z^{\prime}\right)\right]^{*}, \\
& G_{+-}\left(p ; z ; z^{\prime}\right)=\frac{1}{L^{2}} \sum_{j, k}^{\prime} g_{+-}^{j, k} f_{3}^{(j, k)}(z)\left[f_{1}^{(j, k)}\left(z^{\prime}\right)\right]^{*}, \\
& G_{-+}\left(p ; z ; z^{\prime}\right)=\frac{1}{L^{2}} \sum_{j, k}^{\prime} g_{-+}^{j, k} f_{1}^{(j, k)}(z)\left[f_{3}^{(j, k)}\left(z^{\prime}\right)\right]^{*}, \\
& G_{--}\left(p ; z ; z^{\prime}\right)=\frac{1}{L^{2}} \sum_{j, k}^{\prime} g_{--}^{j, k} f_{1}^{(j, k)}(z)\left[f_{1}^{(j, k)}\left(z^{\prime}\right)\right]^{*},
\end{aligned}
$$

which satisfies the boundary conditions implied by Eq. (B.20). If we further use Eq. (2.26) it is easy to see that the ansatz $(\overline{\mathrm{B} .22})$ solves Eq. (B.21) provided

$$
\frac{1}{2}\left(\begin{array}{cc}
p^{2}-\frac{1}{2}(1+\xi) M_{j, k}^{2} & \frac{1}{2}(1-\xi) r_{j, k}^{2} M_{j, k}^{2} \\
\frac{1}{2}(1-\xi) r_{j,-k}^{2} M_{j, k}^{2} & p^{2}-\frac{1}{2}(1+\xi) M_{j, k}^{2}
\end{array}\right)\left(\begin{array}{cc}
g_{++}^{j, k} & g_{+-}^{j, k} \\
g_{-+}^{j, k} & g_{--}^{j, k}
\end{array}\right)=i\left(\begin{array}{ll}
1 & 0 \\
0 & 1
\end{array}\right) .
$$

The solution to this system is

$$
\begin{aligned}
\left(\begin{array}{cc}
g_{++}^{j, k} & g_{+-}^{j, k} \\
g_{-+}^{j, k} & g_{--}^{j, k}
\end{array}\right) & =\frac{2 i}{\left(p^{2}-M_{j, k}^{2}\right)\left(p^{2}-\xi M_{j, k}^{2}\right)}\left(\begin{array}{cc}
p^{2}-\frac{1}{2}(1+\xi) M_{j, k}^{2} & -\frac{1}{2}(1-\xi) r_{j, k}^{2} M_{j, k}^{2} \\
-\frac{1}{2}(1-\xi) r_{j,-k}^{2} M_{j, k}^{2} & p^{2}-\frac{1}{2}(1+\xi) M_{j, k}^{2}
\end{array}\right) \\
& =\left(\begin{array}{cc}
g_{h}^{j, k}+g_{\phi}^{j, k} & -r_{j, k}^{2}\left(g_{h}^{j, k}-g_{\phi}^{j, k}\right) \\
-r_{j,-k}^{2}\left(g_{h}^{j, k}-g_{\phi}^{j, k}\right. & g_{h}^{j, k}+g_{\phi}^{j, k}
\end{array}\right) \\
& =\left(\begin{array}{cc}
r_{j, k} & r_{j, k} \\
-r_{j, k}^{*} & r_{j, k}^{*}
\end{array}\right)\left(\begin{array}{cc}
g_{h}^{j, k} & 0 \\
0 & g_{\phi}^{j, k}
\end{array}\right)\left(\begin{array}{cc}
r_{j, k}^{*} & -r_{j, k} \\
r_{j, k}^{*} & r_{j, k}
\end{array}\right),
\end{aligned}
$$

where we defined

$$
g_{h}^{j, k}=\frac{i}{p^{2}-M_{j, k}^{2}}, \quad g_{\phi}^{j, k}=\frac{i}{p^{2}-\xi M_{j, k}^{2}} .
$$

Eqs. (B.22), (B.24) and (B.25) completely specify the propagator (in mixed position/momentum space) associated with the two degrees of freedom $A_{4}$ and $A_{5}$.

However, one must be careful on how the above propagator, defined as the inverse of the quadratic operator in Eq. (B.19), should be used, since one must impose the constraint $A_{+}=A_{-}^{\dagger}$. Starting with the path integral, one can see that the relation between the various components $G_{++}, G_{+-}, G_{-+}$and $G_{--}$defined in Eq. (B.22) and the tree-level two-point functions that appear in the Feynman rules are

$$
\int d^{4} x e^{i p x}\left\langle A_{+}(x, z) A_{+}^{\dagger}\left(0, z^{\prime}\right)\right\rangle=\frac{1}{2}\left[G_{++}\left(p ; z, z^{\prime}\right)+G_{--}\left(p ; z^{\prime}, z\right)\right],
$$




$$
\begin{aligned}
& \int d^{4} x e^{i p x}\left\langle A_{+}(x, z) A_{+}\left(0, z^{\prime}\right)\right\rangle=\frac{1}{2}\left[G_{+-}\left(p ; z, z^{\prime}\right)+G_{+-}\left(p ; z^{\prime}, z\right)\right], \\
& \int d^{4} x e^{i p x}\left\langle A_{+}^{\dagger}(x, z) A_{+}^{\dagger}\left(0, z^{\prime}\right)\right\rangle=\frac{1}{2}\left[G_{-+}\left(p ; z, z^{\prime}\right)+G_{-+}\left(p ; z^{\prime}, z\right)\right], \\
& \int d^{4} x e^{i p x}\left\langle A_{+}^{\dagger}(x, z) A_{+}\left(0, z^{\prime}\right)\right\rangle=\frac{1}{2}\left[G_{--}\left(p ; z, z^{\prime}\right)+G_{++}\left(p ; z^{\prime}, z\right)\right] .
\end{aligned}
$$

Here we wrote all correlators in terms of $A_{+}$by using $A_{-}=A_{+}^{\dagger}$. The last two relations are simply the complex conjugates of the first two. Projecting, for example, the second relation on the momentum space wavefunctions (2.3), one gets (we indicate only the dependence on the extra dimensional momenta)

$$
\begin{aligned}
\left\langle A_{+}^{m, l} A_{+}^{m^{\prime}, l^{\prime}}\right\rangle & =\frac{1}{2}\left[G_{+-}^{\left(m, l ; m^{\prime}, l^{\prime}\right)}+G_{+-}^{\left(-m^{\prime},-l^{\prime} ;-m,-l\right)}\right] \\
& =\frac{1}{2}\left[\tilde{g}_{+-}^{m, l} \hat{\delta}\left(m, l ; m^{\prime}, l^{\prime} ; 1\right)+\tilde{g}_{+-}^{-m,-l} \hat{\delta}\left(-m,-l ;-m^{\prime},-l^{\prime} ; 3\right)\right] \\
& =\frac{1}{2}\left[\tilde{g}_{+-}^{m, l}+\tilde{g}_{+-}^{-m,-l}\right] \hat{\delta}\left(m, l ; m^{\prime}, l^{\prime} ; 1\right),
\end{aligned}
$$

where we used Eq. (A.9). The remaining relations in Eq. (B.26) can be similarly expressed in terms of

$$
\begin{aligned}
G_{++}^{\left(m, l ; m^{\prime}, l^{\prime}\right)} & =\left(g_{h}^{m, l}+g_{\phi}^{m, l}\right) \hat{\delta}\left(m, l ; m^{\prime}, l^{\prime} ; 3\right), \\
G_{+-}^{\left(m, l ; m^{\prime}, l^{\prime}\right)} & =-r_{m, l}^{2}\left(g_{h}^{m, l}-g_{\phi}^{m, l}\right) \hat{\delta}\left(m, l ; m^{\prime}, l^{\prime} ; 1\right), \\
G_{-+}^{\left(m, l ; m^{\prime}, l^{\prime}\right)} & =-r_{m, l}^{* 2}\left(g_{h}^{m, l}-g_{\phi}^{m, l}\right) \hat{\delta}\left(m, l ; m^{\prime}, l^{\prime} ; 3\right), \\
G_{--}^{\left(m, l ; m^{\prime}, l^{\prime}\right)} & =\left(g_{h}^{m, l}+g_{\phi}^{m, l}\right) \hat{\delta}\left(m, l ; m^{\prime}, l^{\prime} ; 1\right),
\end{aligned}
$$

which follow from Eq. (A.9), noting that from the definition of $\tilde{g}_{+-}^{m, l}$, Eq. (A.4) with $n_{1}=3$ and $n_{2}=1$, and the explicit expression for $g_{+-}^{m, l}$ in Eq. ( $\left.\mathrm{B.24}\right)$, one finds $\tilde{g}_{+-}^{m, l}=g_{+-}^{m, l}$ and similarly $\tilde{g}_{-+}^{m, l}=g_{-+}^{m, l}, \tilde{g}_{++}^{m, l}=g_{++}^{m, l}$ and $\tilde{g}_{--}^{m, l}=g_{--}^{m, l}$. In Eq. (B.28) we also used the definitions (B.25).

\section{B.4 Feynman Rules for Gauge Interactions}

We finally present the Feynman rules in momentum space for the interactions among fermions and gauge fields in six dimensions. These can be read directly from the vertices derived in [11, and we simply present them diagramaticaly in Figures 11 and 12.

\section{References}

[1] G. Servant and T. M. P. Tait, "Is the lightest Kaluza-Klein particle a viable dark matter candidate?," Nucl. Phys. B 650 (2003) 391 [arXiv:hep-ph/0206071]; "Elastic scattering and direct detection of Kaluza-Klein dark matter," New J. Phys. 4 (2002) 99 [arXiv:hep-ph/0209262].

H. C. Cheng, J. L. Feng and K. T. Matchev, "Kaluza-Klein dark matter," Phys. Rev. Lett. 89, 211301 (2002) [arXiv:hep-ph/0207125]. 


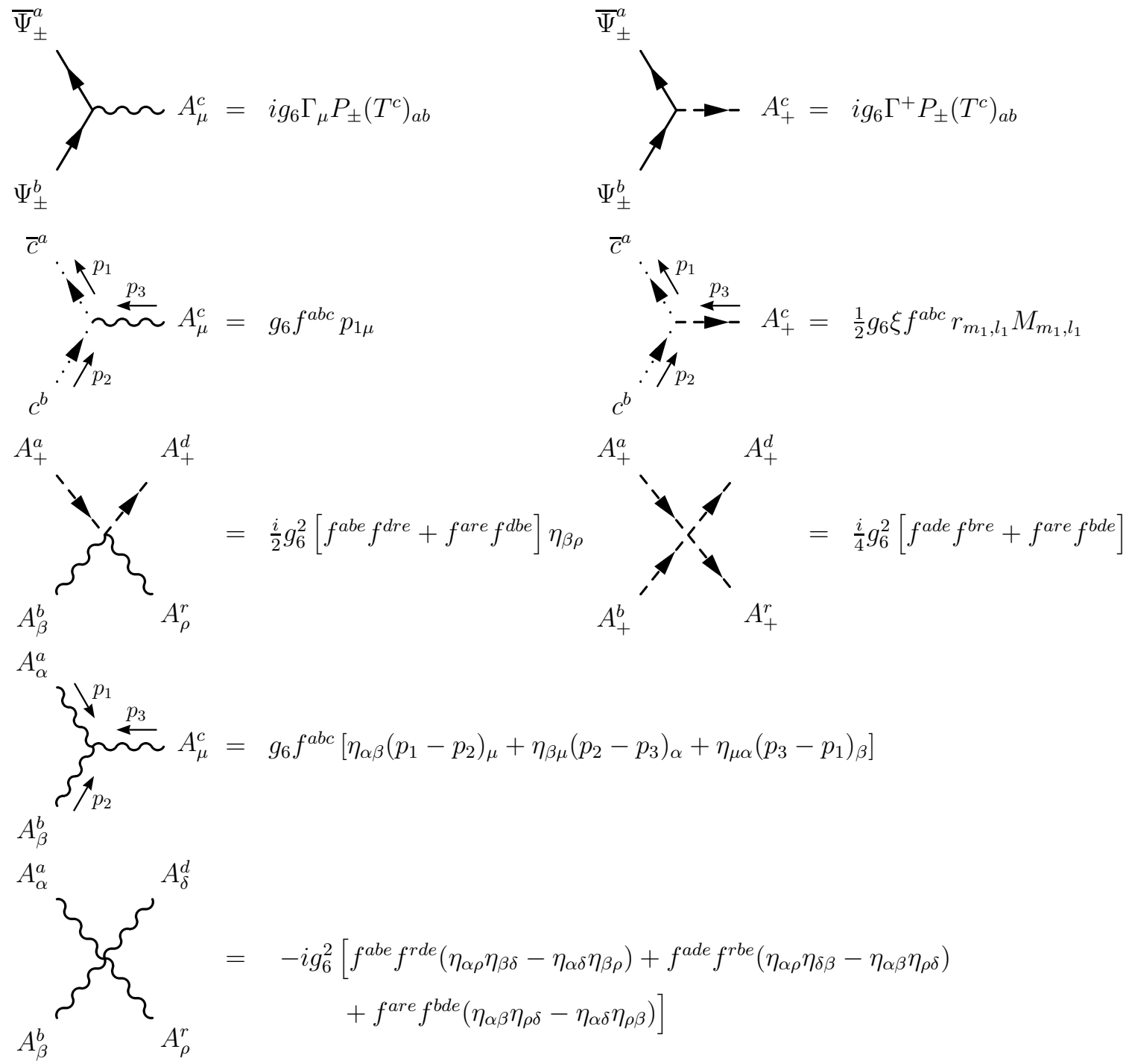

Figure 11: Feynman rules in the 6D gauge sector, including the additional scalar degrees of freedom. The dashed lines with the arrow represent the propagation of $A_{+}$. For the interaction of two fermions or two ghosts with an outgoing $A_{+}$there is a corresponding vertex with an incoming $A_{+}$. The corresponding rules are obtained by $\Gamma^{+} \rightarrow \Gamma^{-}$, where $\Gamma^{ \pm}=\frac{1}{2}\left(\Gamma^{4} \pm i \Gamma^{5}\right)$, and $r_{m, l} \rightarrow r_{m, l}^{*}$, respectively. The $r_{m, l}$ phases were defined in Eq. (2.27).

J. L. Feng, A. Rajaraman and F. Takayama, "Superweakly-interacting massive particles," Phys. Rev. Lett. 91, 011302 (2003) [arXiv:hep-ph/0302215];

"Graviton cosmology in universal extra dimensions," Phys. Rev. D 68, 085018 (2003) [arXiv:hep-ph/0307375].

K. Kong and K. T. Matchev, arXiv:hep-ph/0509119.

[2] B. A. Dobrescu and E. Poppitz, "Number of fermion generations derived from anomaly 


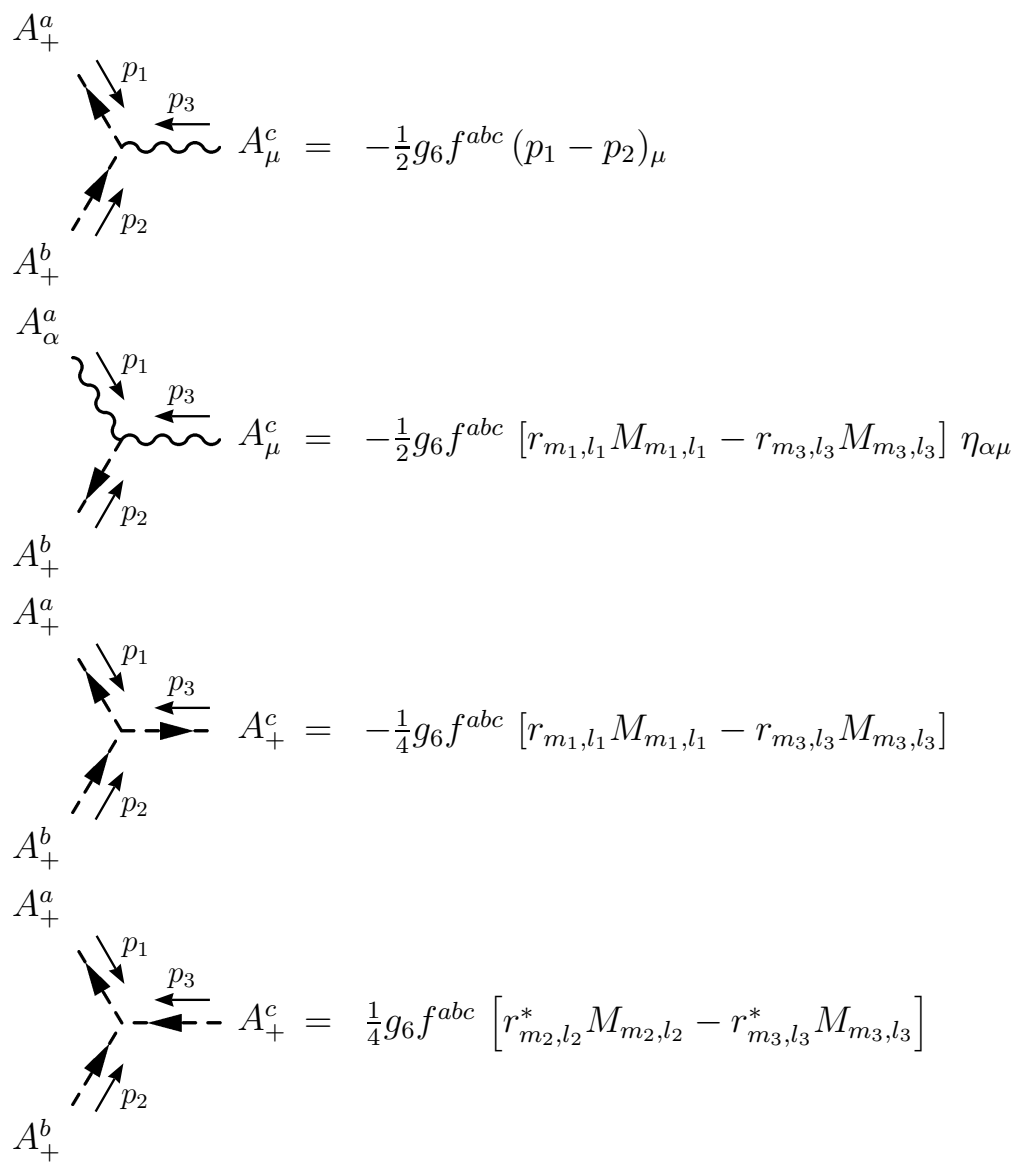

Figure 12: Feynman rules in the 6D gauge sector, including the additional scalar degrees of freedom. The dashed lines with the arrow represent the propagation of $A_{+}$. For the interaction of two gauge bosons with a single outgoing $A_{+}$there is a corresponding vertex with an incoming $A_{+}$. The corresponding rule is obtained by the replacement $r_{m, l} \rightarrow r_{m, l}^{*}$. The $r_{m, l}$ phases were defined in Eq. (2.27).

cancellation," Phys. Rev. Lett. 87, 031801 (2001) [arXiv:hep-ph/0102010].

N. Borghini, Y. Gouverneur and M. H. G. Tytgat, "Anomalies and fermion content of grand unified theories in extra dimensions," Phys. Rev. D 65, 025017 (2002) [arXiv:hep-ph/0108094]. M. Fabbrichesi, R. Percacci, M. Piai and M. Serone, "Cancellation of global anomalies in spontaneously broken gauge theories," Phys. Rev. D 66, 105028 (2002) [arXiv:hep-th/0207013].

[3] T. Appelquist, B. A. Dobrescu, E. Pontón and H. U. Yee, "Proton stability in six dimensions," Phys. Rev. Lett. 87, 181802 (2001) [arXiv:hep-ph/0107056].

[4] T. Appelquist, B. A. Dobrescu, E. Pontón and H. U. Yee, "Neutrinos vis-a-vis the six-dimensional standard model," Phys. Rev. D 65, 105019 (2002) [arXiv:hep-ph/0201131];

[5] M. B. Green, J. H. Schwarz and P. C. West, "Anomaly Free Chiral Theories In Six-Dimensions," Nucl. Phys. B 254, 327 (1985).

K. R. Dienes, E. Dudas and T. Gherghetta, "Grand unification at intermediate mass scales through extra dimensions," Nucl. Phys. B 537, 47 (1999) [arXiv:hep-ph/9806292]. 
N. Arkani-Hamed, H. C. Cheng, B. A. Dobrescu and L. J. Hall, "Self-breaking of the standard model gauge symmetry," Phys. Rev. D 62, 096006 (2000) [arXiv:hep-ph/0006238]. M. Hashimoto, M. Tanabashi and K. Yamawaki, "Top mode standard model with extra dimensions," Phys. Rev. D 64, 056003 (2001) [arXiv:hep-ph/0010260].

G. R. Dvali, S. Randjbar-Daemi and R. Tabbash, "The origin of spontaneous symmetry breaking in theories with large extra dimensions," Phys. Rev. D 65, 064021 (2002) [arXiv:hep-ph/0102307].

A. Hebecker and J. March-Russell, "The structure of GUT breaking by orbifolding," Nucl. Phys. B 625, 128 (2002) [arXiv:hep-ph/0107039].

A. Hebecker and M. Ratz, "Group-theoretical aspects of orbifold and conifold GUTs," Nucl. Phys. B 670, 3 (2003) [arXiv:hep-ph/0306049].

M. Fabbrichesi, M. Piai and G. Tasinato, "Axion and neutrino physics from anomaly cancellation," Phys. Rev. D 64, 116006 (2001) [arXiv:hep-ph/0108039].

L. J. Hall, Y. Nomura, T. Okui and D. R. Smith, "SO(10) unified theories in six dimensions," Phys. Rev. D 65, 035008 (2002) [arXiv:hep-ph/0108071]. J. M. Frere, M. V. Libanov and

S. V. Troitsky, "Neutrino masses with a single generation in the bulk," JHEP 0111, 025 (2001) [arXiv:hep-ph/0110045].

R. N. Mohapatra and A. Perez-Lorenzana, "Neutrino mass, proton decay and dark matter in TeV scale universal extra dimension models," Phys. Rev. D 67, 075015 (2003) [arXiv:hep-ph/0212254]. T. Watari and T. Yanagida, "Higher dimensional supersymmetry as an origin of the three families for quarks and leptons," Phys. Lett. B 532, 252 (2002) [arXiv:hep-ph/0201086].

T. Asaka, W. Buchmuller and L. Covi, "Bulk and brane anomalies in six dimensions," Nucl. Phys. B 648, 231 (2003) [arXiv:hep-ph/0209144]; "Quarks and leptons between branes and bulk," Phys. Lett. B 563, 209 (2003) [arXiv:hep-ph/0304142].

C. Biggio, F. Feruglio, I. Masina and M. Perez-Victoria, "Fermion generations, masses and mixing angles from extra dimensions," Nucl. Phys. B 677, 451 (2004) [arXiv:hep-ph/0305129].

C. A. Scrucca, M. Serone, L. Silvestrini and A. Wulzer, JHEP 0402, 049 (2004) [arXiv:hep-th/0312267].

S. Gabriel, S. Nandi and G. Seidl, "6D Higgsless standard model," Phys. Lett. B 603, 74 (2004) [arXiv:hep-ph/0406020].

[6] H. C. Cheng, K. T. Matchev and M. Schmaltz, "Radiative corrections to Kaluza-Klein masses," Phys. Rev. D 66, 036005 (2002) [arXiv:hep-ph/0204342]; "Bosonic supersymmetry? Getting fooled at the LHC," Phys. Rev. D 66, 056006 (2002) [arXiv:hep-ph/0205314].

A. Datta, K. Kong and K. T. Matchev, "Discrimination of supersymmetry and universal extra dimensions at hadron Phys. Rev. D 72, 096006 (2005) [Erratum-ibid. D 72, 119901 (2005)] [arXiv:hep-ph/0509246].

[7] T. Appelquist, H. C. Cheng and B. A. Dobrescu, "Bounds on universal extra dimensions," Phys. Rev. D 64, 035002 (2001) [arXiv:hep-ph/0012100].

T. Appelquist and H. U. Yee, "Universal extra dimensions and the Higgs boson mass," Phys. Rev. D 67, 055002 (2003) [arXiv:hep-ph/0211023].

J. F. Oliver, J. Papavassiliou and A. Santamaria, "Universal extra dimensions and Z $\rightarrow$ b anti-b," Phys. Rev. D 67, 056002 (2003) [arXiv:hep-ph/0212391].

[8] H. Georgi, A. K. Grant and G. Hailu, "Brane couplings from bulk loops," Phys. Lett. B 506, 207 (2001) [arXiv:hep-ph/0012379].

[9] E. Pontón and E. Poppitz, "Casimir energy and radius stabilization in five and six dimensional orbifolds," JHEP 0106, 019 (2001) [arXiv:hep-ph/0105021]. M. Carena, T. M. P. Tait and 
C. E. M. Wagner, "Branes and orbifolds are opaque," Acta Phys. Polon. B 33, 2355 (2002)

[arXiv:hep-ph/0207056].

H. Davoudiasl, J. L. Hewett and T. G. Rizzo, "Brane localized kinetic terms in the

Randall-Sundrum model," Phys. Rev. D 68, 045002 (2003) [arXiv:hep-ph/0212279].

M. Carena, E. Pontón, T. M. P. Tait and C. E. M. Wagner, Phys. Rev. D 67, 096006 (2003)

[arXiv:hep-ph/0212307]. M. Carena, A. Delgado, E. Pontón, T. M. P. Tait and C. E. M. Wagner,

"Precision electroweak data and unification of couplings in warped extra Phys. Rev. D 68, 035010

(2003) [arXiv:hep-ph/0305188]; "Warped fermions and precision tests," Phys. Rev. D 71, 015010

(2005) [arXiv:hep-ph/0410344].

F. del Aguila, M. Perez-Victoria and J. Santiago, "Bulk fields with brane terms," Eur. Phys. J. C 33, S773 (2004) [arXiv:hep-ph/0310352].

[10] B. A. Dobrescu and E. Pontón, "Chiral compactification on a square," JHEP 0403, 071 (2004) [arXiv:hep-th/0401032].

[11] G. Burdman, B. A. Dobrescu and E. Pontón, "Six-dimensional gauge theory on the chiral square," arXiv:hep-ph/0506334.

[12] C. Csaki, C. Grojean and H. Murayama, "Standard model Higgs from higher dimensional gauge fields," Phys. Rev. D 67, 085012 (2003) [arXiv:hep-ph/0210133].

[13] M. Hashimoto and D. K. Hong, "Topcolor breaking through boundary conditions," Phys. Rev. D 71, 056004 (2005) [arXiv:hep-ph/0409223].

M. Hashimoto, "Topcolor model in extra dimensions and nontrivial boundary conditions," arXiv:hep-ph/0502242.

[14] G. Burdman, B. A. Dobrescu and E. Pontón, in preparation.

[15] A. Manohar and H. Georgi, "Chiral Quarks And The Nonrelativistic Quark Model," Nucl. Phys. B 234, 189 (1984); M. A. Luty, "Naive dimensional analysis and supersymmetry," Phys. Rev. D 57, 1531 (1998) [arXiv:hep-ph/9706235]. A. G. Cohen, D. B. Kaplan and A. E. Nelson, "Counting 4pi's in strongly coupled supersymmetry," Phys. Lett. B 412, 301 (1997) [arXiv:hep-ph/9706275];

[16] Z. Chacko, M. A. Luty and E. Pontón, "Massive higher-dimensional gauge fields as messengers of supersymmetry JHEP 0007, 036 (2000) [arXiv:hep-ph/9909248]. 